\title{
On annular maps of the torus and sublinear diffusion
}

\author{
Pablo Dávalos
}

\begin{abstract}
There is a classification by Misiurewicz and Ziemian MZ89 of elements in Homeo $\left(\mathbf{T}^{2}\right)$ by their rotation set $\rho$, according to wether $\rho$ is a point, a segment or a set with nonempty interior. A recent classification of nonwandering elements in $\operatorname{Homeo}_{0}\left(\mathbf{T}^{2}\right)$ by Koropecki and Tal was given in $[\mathrm{KT}$, according to the itrinsic underlying ambient where the dynamics takes place: planar, annular and strictly toral maps. We study the link between these two classifications, showing that, even abroad the nonwandering setting, annular maps are characterized by rotation sets which are rational segments. Also, we obtain information on the sublinear diffusion of orbits in the -not very well understood- case that $\rho$ has nonempty interior.
\end{abstract}

\section{Contents}

1 Introduction 2

2 Preliminaries 6

2.1 Notations. . . . . . . . . . . . . . . . . . . . . . . 6

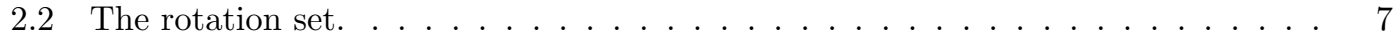

2.3 Atkinson's Lemma. . . . . . . . . . . . . . . . . . . . . . . 9

2.4 A theorem of Handel . . . . . . . . . . . . . . . . . . . . . . 9

2.5 Stable and unstable sets, in the case there is a Brouwer curve. . . . . . . . . . . 10

2.6 Brouwer Theory . . . . . . . . . . . . . . . . . . 11

2.7 Poincaré-Bendixson theory. . . . . . . . . . . . . . . . . . 13

2.8 Intersection number of certain lines. . . . . . . . . . . . . . . . . 14

3 Theorems A, B and C. 14

4 Proof of Theorem D. 15

4.1 About inessential sets and the $\operatorname{set} \operatorname{sing}(\mathcal{F}) \ldots \ldots \ldots \ldots \ldots$

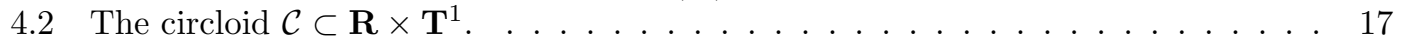

4.3 Definition of the bound $M=M(f)$ from Theorem D. . . . . . . . . . . 19

4.4 Collapsing the connected components of $\operatorname{sing}(\widehat{\mathcal{F}}) \ldots \ldots \ldots \ldots \ldots$

4.5 Case that $\mathcal{C}$ does not contain singularities. . . . . . . . . . . 20

4.6 Case that $\mathcal{C}$ contains singularities . . . . . . . . . . . . . . 32

4.7 Construction of the $\operatorname{arc} \delta$; proof of Lemma 4.25 . . . . . . . . . . . . 51

\section{References}

This work was partially supported by FAPESP-Brasil and ITESO-México. 


\section{Introduction}

A very useful tool for understanding the dynamics of a homeomorphism $f$ of a surface $M$ in the isotopy class of the identity is the rotation set; a topological invariant which is a subset of $H_{1}(M, \mathbf{R})$ and roughly consists of asymptotic homological velocity vectors of points under iteration.

It was first introduced by H. Poincaré Poi52] as the rotation number of circle homeomorphisms, and in this case he proved that, from the topological point of view, the rotation number leads to a complete classification of the dynamics. The concept was later extended for homeomorphisms of any manifold Sch57, MZ89, Pol92, Cal05] or even for metric spaces Mat97. For the case of surfaces there exist results relating the dynamics of a homeomorphism with its rotation set; for example showing that certain vectors with rational coordinates in the rotation set imply the existence of periodic orbits for $f$ [Fra95, Mat97, and showing that 'large' rotation sets have positive topological entropy LM91, Mat97.

In this article we deal with the case $M=\mathbf{T}^{2}$. For any lift $\widehat{f}: \mathbf{R}^{2} \rightarrow \mathbf{R}^{2}$ of $f$, the rotation set of $\widehat{f}$, denoted $\rho(\widehat{f})$, is defined as the set of accumulation points of sequences of the form

$$
\left(\frac{\widehat{f}^{n_{i}}\left(x_{i}\right)-x_{i}}{n_{i}}\right)_{i \in \mathbf{N}}
$$

where $m_{i} \rightarrow \infty$ and $x_{i} \in \mathbf{R}^{2}$, and it is known to be a compact, convex subset of $H_{1}\left(\mathbf{T}^{2}, \mathbf{R}\right) \simeq \mathbf{R}^{2}$ MZ89. An interesting fact about the rotation set is that it prompts a classification of the set Homeo $_{0}\left(\mathbf{T}^{2}\right)$ of homeomorphisms of $\mathbf{T}^{2}$ homotopic to the identity into three (disjoint) cases, depending wether the rotation set is:

(i) a set consisting of a single point;

(ii) a compact segment;

(iii) a set with nonempty interior.

In case $(i)$ we say that $f$ is a pseudorotation. Pseudorotations have been thoroughly studied. For example, in [Jäg09b] it is given a Poincaré-like classification theorem for conservative pseudorotations, and in KT12b a classification theorem is given for rational pseudorotations, that is, for the case that $\rho(\widehat{f})$ is a single rational vector. Also, in [KT12a, Fay02, BCJR09] are constructed examples with exotic dynamical properties.

In case (iii) it is known that $f$ must have positive entropy [LM91], any vector in $\rho(\widehat{f})$ is realized by a minimal set MZ89, and any rational vector in the interior of $\rho(\widehat{f})$ is realized by a periodic orbit Fra95. Thus, an interesting mechanism for creating entropy and (infinitely many) periodic orbits is given by the creation of (at least three) points with non-colinear asymptotic velocity vectors. Worth to mention, the only known examples in case (iii) are polygons or "infinite polygons" with a countable set of extremal points Kwa92, Kwa95.

For case (ii) it is conjectured in FM90, that the only possible examples are rational segments, meaning segments of rational slope containing rational vectors, and segments of irrational slope containing a rational endpoint. For the latter case, an example in [Han89] attributed to Katok gives essentially the unique mechanism known to the author to produce examples with such a rotation set. Examples with rational segments are an analog of twist maps for elements in Homeo $_{0}\left(\mathbf{T}^{2}\right)$, and they have been recently studied. For example, results concerning dynamical models and the existence of periodic orbits associated to any rational vector in $\rho(\widehat{f})$ include KK08, GKT12, Dáv13]. 
Aside this classification given by the rotation set, a recent new classification for the nonwandering elements in $\mathrm{Homeo}_{0}\left(\mathbf{T}^{2}\right)$ was given in $[\mathrm{KT}$. In that article, it is shown that any nonwandering element of $\operatorname{Homeo}_{0}\left(\mathbf{T}^{2}\right)$ falls in one of the following categories:

(a) there is $k \in \mathbf{N}$ such that $\operatorname{Fix}\left(f^{k}\right)$ is fully essential, that is, $\mathbf{T}^{2} \backslash \operatorname{Fix}\left(f^{k}\right)$ is a union of topological open discs.

(b) $f$ is annular: there is $k \in \mathbf{N}$ and a lift $F: \mathbf{R}^{2} \rightarrow \mathbf{R}^{2}$ of $f^{k}$ such that the deviations in the direction of some nonzero $v \in \mathbf{Z}^{2}$ are uniformly bounded:

$$
-M \leq\left\langle F^{n}(x)-x, v\right\rangle \leq M \quad \text { for all } x \in \mathbf{R}^{2} \text { and } n \in \mathbf{Z} .
$$

(c) $f$ is strictly toral. This roughly means that the dynamics of $f$ cannot be embedded in the plane or the annulus, and also, the 'irrotational' part of the dynamics is contained in 'elliptic islands' (below we explain this concept precisely).

In case $(a)$ the dynamics of $f$ takes place essentially in the plane, as the set $\mathbf{T}^{2} \backslash \operatorname{Fix}\left(f^{k}\right)$ can be embedded in the plane.

In case $(b)$ it is easy to see that there is a finite covering of $\mathbf{T}^{2}$ such that the lift of $f$ to this covering has an invariant annular set (see for example Remark 3.10 in Jäg09a ), so that in some sense the dynamics of $f$ in a finite covering is embedded in an annulus. The notion of annular homeomorphism is equivalent to saying that $f$ is rationally bounded in the sense of Jäg09a.

For case $(c)$, let us first explain the notion of essential points for a nonwandering homeomorphism. An essential set $K \subset \mathbf{T}^{2}$ is a set which is not contained in a topological open disc. A point $x \in \mathbf{T}^{2}$ is essential for $f$ if the orbit of every neighborhood of $f$ is an essential subset of $\mathbf{T}^{2}$. Roughly speaking, this means that $x$ has a weak form of rotational recurrence. The set of essential points for $f$ is denoted $\operatorname{Ess}(f)$, and the set of inessential points is $\operatorname{Ine}(f)=\mathbf{T}^{2} \backslash \operatorname{Ess}(f)$. The precise statement of item $(c)$ is the following: the set $\operatorname{Ess}(f)$ is nonempty, connected and fully essential, and Ine $(f)$ is the union of pairwise disjoint bounded open discs. Therefore, if $f$ is strictly toral, there is a decomposition of the dynamics into a union Ine $(f)$ (possibly empty) of periodic bounded discs which can be regarded as "elliptic islands", and a fully essential set $\operatorname{Ess}(f)$ which carries the "rotational" part of the dynamics.

Case $(c)$ is disjoint from cases $(a)$ and $(b)$. However, cases $(a)$ and $(b)$ intersect (the identity belongs to both). In order to distinguish "planar from annular" we make the following definition. We say that a homeomorphism $f$ is planar if either $f$ belongs to case $(a)$ or the orbits of (any lift to $\mathbf{R}^{2}$ of) $f$ are uniformly bounded. Also, we say that $f$ is strong annular if $f$ is annular and is not planar.

We therefore have that the set of nonwandering elements of $\operatorname{Homeo}_{0}\left(\mathbf{T}^{2}\right)$ is a disjoint union of planar, strong annular and strictly toral maps.

There exist some links of course between these two classifications in the nonwandering case. Let us see how cases $(i),($ ii $),($ iii $)$ fall into the categories of planar, strong annular and strictly toral.

For case $(i i i)$, namely if $\rho(\widehat{f})$ has nonempty interior, it is clear that $f$ cannot be annular. It cannot either be planar, as in [KT] it is shown that planar maps are irrotational: $\rho(\widehat{f})=\{(0,0)\}$. Therefore in case $($ iii $) f$ is strictly toral.

As for case $(i)$, an irrational pseudorotation (that is, when $\rho(\widehat{f})$ is a totally irrational vector) is analogously seen to be strictly toral. If $f$ is a rational pseudorotation, then $f$ might be either planar (e.g. the identity), strictly toral (see $\S 1.2$ in KT12b for an exampl $\ell^{1}$, and it is not

\footnotetext{
${ }^{1}$ Such example is a non-wandering map with a unique invariant measure supported on a fixed point. In contrast, in Tal13] it is shown that an irrational pseudorotation which preserves a measure with full suport cannot be strictly toral.
} 
known wether $f$ may be strong annular (see Question 3 in [Tal13]). Finally, if $\rho(\widehat{f})$ is a vector which is neither rational nor totally irrational, then $f$ may be either strong annular (e.g. a rigid translation) or strictly toral (as is Furstenberg's example [Fur61]).

The options for case (ii) are either strong annular or strictly toral. As we mentioned above, the only known examples are rational segments and segments with irrational slope containing a rational endpoint. Note that if any other example may exist, it cannot be annular and therefore it must be strictly toral. The case of a segment with irrational slope containing a rational endpoint is also strictly toral, and rational segments may of course be realized by annular maps (e.g. the twist-like map $(x, y) \mapsto(x, y+\sin (2 \pi x)))$. The question wether rational segments may or may not be realized by strictly toral maps remained open.

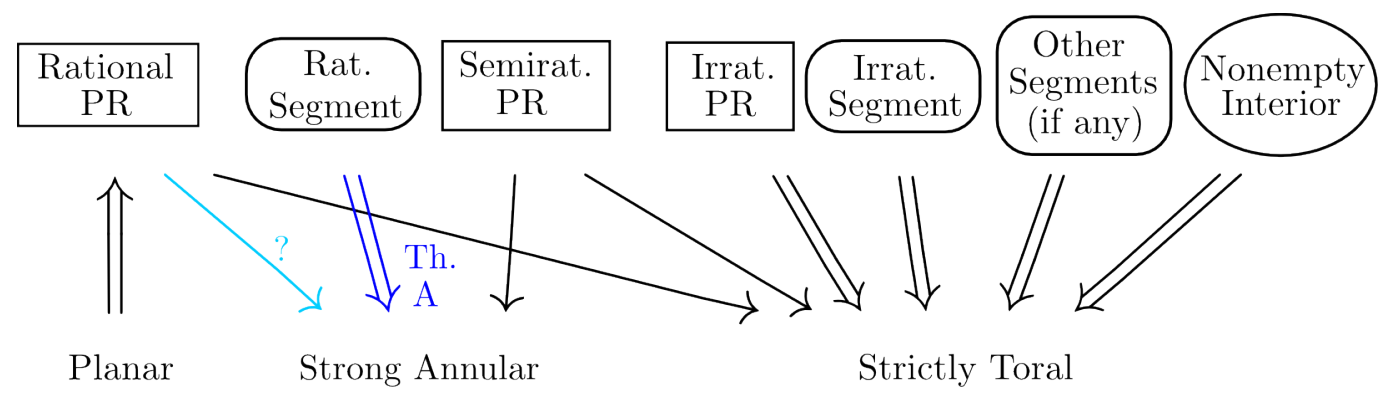

Figure 1: Link between the classifications of Misiurewicz-Ziemian and Koropecki-Tal. Double arrows represent implications, and single arrows existence of examples. "Semirat. PR" stands for the pseudorotations which are neither rational nor irrational.

Summarizing, the only two remaining open questions in this direction are the following:

1. If $f$ is a non-wandering pseudorotation, can $f$ be strong annular?

2. If $\rho(\widehat{f})$ is a rational segment, can $f$ be strictly toral?

A negative answer to Question 2 was given in [GKT12] in the area preserving case.

One of the objectives of this work is to contrubute to understanding the link between these two classifications by giving a negative answer to Question 2, even abroad the nonwandering setting, namely, for any element of $\mathrm{Homeo}_{0}\left(\mathbf{T}^{2}\right)$. This is given by the following.

Theorem A. If $\rho(\widehat{f})$ is a segment with rational slope containing rational points, then $f$ is annular.

Another purpose of this article is to give a (very small) step in understanding case (iii); the case when $\rho(\widehat{f})$ has nonempty interior. As we said, the only known examples are polygons or "infinite polygons" with a countable set of extremal points. It is also not known if there are convex subsets of $\mathbf{R}^{2}$ which are not realizable as rotation sets. The next theorem gives us information about the sublinear diffusion of displacements away from the rotation set, in the case that $\partial \rho(\widehat{f}) \subset \mathbf{R}^{2}$ contains a rational segment $S$. Note that without loss of generality we may assume that the segment $S$ is contained in a line with rational slope passing through the origin in $\mathbf{R}^{2}$ (see Section 2.2 for the basic properties of the rotation set), and the next theorem then tells us that displacements must be uniformly bounded in the direction orthogonal to $S$ and outwards $\rho(\widehat{f})$. 
Theorem B. If $\rho(\widehat{f})$ is contained in the half-plane $\left\{x \in \mathbf{R}^{2}:\langle x, v\rangle \leq 0\right\}$, for some $v \in \mathbf{Q}^{2}$, and if the line $v^{\perp}$ contains more than one point of $\rho(\widehat{f})$, then there exists $M>0$ such that

$$
\left\langle\widehat{f}^{n}(x)-x, v\right\rangle<M \quad \text { for all } x \in \mathbf{R}^{2} \text { and } n \in \mathbf{N} .
$$

Finally, we adress the following question: to what extent does the rotation set capture the rotation information? In [KT12a is constructed a (smooth, Lebesgue-ergodic) example with $\rho(\widehat{f})=\{(0,0)\}$ and such that almost every point rotates at a sublinear speed in every direction, that is, for almost every $x \in \mathbf{R}^{2}$, the sequence

$$
\left(\frac{\widehat{f}^{n}(x)-x}{\left|\widehat{f}^{n}(x)-x\right|}\right)_{n \in \mathbf{N}}
$$

accumulates in the whole circle $\mathbf{T}^{1}$. In other words, the dynamics is far away to be rotationless, even though $\rho(\widehat{f})$ is reduced to the point $\{(0,0)\}$. This phenomenon of sublinear diffusion may also occur for any pseudorotation KK09, Jäg09a.

For larger rotation sets the situation turns out to be quite different. For example, Theorem A tells us that if $\rho(\widehat{f})$ is a rational segment, then there is no sublinear diffusion in the direction perpendicular to $\rho(\widehat{f})$. Moreover, if $\rho(\widehat{f})$ is a rational polygon, that is, a non-degenerate polygon whose extremal points are rational vectors, we will show that there is no sublinear diffusion at all (note that any rational polygon is realized as a rotation set [Kwa92]). This is given by the following.

Theorem C. If $\rho(\widehat{f})$ is a non-degenerate polygon with rational endpoints, then there exists $M>0$ such that

$$
\sup _{x \in \mathbf{R}^{2}, n \in \mathbf{N}} d\left(\widehat{f}^{n}(x)-x, n \rho(\widehat{f})\right)<M .
$$

We make the important remark that in the last three theorems, the bound $M=M(f)>0$ in them will be constructed explicitly. Once the constant $M(f)$ is constructed, we will prove the following main theorem, which will have as corollaries Theorems A, B and C. It gives us our main dichotomy between bounded mean motion and positive linear speed.

Theorem D. Suppose that $\rho(\widehat{f})$ contains two vectors $(0, a)$ and $(0, b)$, with $a<0<b$.

Then, one of the following holds:

- $\sup _{x \in \mathbf{R}^{2}, n \in \mathbf{N}} \operatorname{pr}_{1}\left(\widehat{f}^{n}(x)-x\right)<M(f)$;

- $\exists x \in \mathbf{R}^{2} \exists N>0$ s.t. $\operatorname{pr}_{1}\left(\widehat{f}^{n N}(x)-x\right)>n \quad \forall n \in \mathbf{N}$.

This article is organized as follows. In Section 2 we introduce some basic facts about the rotation set, Atkinson's Lemma from ergodic theory, Brouwer theory, Poincaré-Bendixson theory and a theorem of Handel. In Section 3 we prove Theorems A, B and C from Theorem D, and Section 4 is devoted to the proof of Theorem D.

Acknowledgments. I am very grateful to F. A. Tal for carefully listening to the proof of the main theorem, for improvements and for key suggestions. I am also grateful to F. Le Roux and P. Le Calvez for their hospitality during a short visit to Université Paris VII and for suggestions which led to simplifications in the proof of the main theorem. Finally, I also thank T. Jäger for suggestions that led to improvements and A. Koropecki , A. Passeggi and J. Xavier for useful conversations. 


\section{Preliminaries}

\section{$2.1 \quad$ Notations.}

We denote $\mathbf{N}$ the set of positive integers, and $\mathbf{N}_{0}=\mathbf{N} \cup\{0\}$. Also we denote the circle $\mathbf{T}^{1}=\mathbf{R} / \mathbf{Z}$ and the two-torus $\mathbf{T}^{2}=\mathbf{R}^{2} / \mathbf{Z}$. If $S=\mathbf{R} \times \mathbf{T}^{1}$ or $\mathbf{R}^{2}$, we will denote the translation $T_{1}: S \rightarrow S$, $T_{1}(x, y)=(x+1, y)$, and $T_{2}: \mathbf{R}^{2} \rightarrow \mathbf{R}^{2}$ denotes $T_{2}(x, y)=(x, y+1)$.

If $S$ is any surface, a map $\gamma:[a, b] \rightarrow S$ and its image will be both referred to as a curve. By an arc we mean a simple compact curve $\gamma:[0,1] \rightarrow S$. For the concatenation of two arcs $\gamma_{1}, \gamma_{2}:[0,1] \rightarrow S$ we will use the 'left-to-right' notation

$$
\gamma_{1} \cdot \gamma_{2}= \begin{cases}\gamma_{1}(2 t) & t \in[0,1 / 2] \\ \gamma_{2}(2 t-1) & t \in[1 / 2,1]\end{cases}
$$

The arc $\gamma$ parametrized with the opposite orientation will be denoted by $\gamma^{-1}$. If $S=\mathbf{R} \times \mathbf{T}^{1}$ or $\mathbf{T}^{2}$, a vertical curve $\gamma \subset S$ is a loop which has homotopy class $(0, n) \in H_{1}(S, \mathbf{Z})$, for some $n \in \mathbf{Z}$.

For an isotopy $\left(f_{t}\right)_{t}$ on a surface $S$, define its canonical lift to a covering space $\widetilde{S}$ as the lift $\left(\widetilde{f}_{t}\right)_{t}$ such that $\widetilde{f}_{0}=$ Id. For the homeomorphism $f=f_{1}$, we call the canonical lift of $f$ with respect to the isotopy $\left(f_{t}\right)_{t}$ as the lift $\widetilde{f}=\widetilde{f}_{1}$. If the isotopy between Id and $f$ is implicitly known, we just refer to the canonical lift of $f$. If $\left(f_{t}\right)_{t \in[0,1]}$ is an isotopy, we will denote also by $\left(f_{t}\right)_{t}$ its extension for $t \in \mathbf{R}$, namely $f_{t}=f_{t} \bmod 1 \circ f^{\lfloor t\rfloor}$, where we use the notation

$$
t \quad \bmod 1=t-\lfloor t\rfloor \text { and }\lfloor t\rfloor= \begin{cases}\lfloor t\rfloor & t \geq 0 \\ -\lfloor|t|\rfloor & t<0\end{cases}
$$

If $X \subset S$, two $\operatorname{arcs} \gamma_{1}, \gamma_{2} \subset S \backslash X$ are said to be homotopic $\operatorname{Rel}(X)$ if there is a homotopy on $S \backslash X$ from $\gamma_{1}$ to $\gamma_{2}$. If $\gamma_{1}$ and $\gamma_{2}$ are homotopic with fixed endpoints, we will abreviate this by saying that $\gamma_{1}, \gamma_{2}$ are homotopic wfe. The interior of an $\operatorname{arc} \gamma$, denoted $\operatorname{int}(\gamma)$ is defined as $\gamma \backslash\{\gamma(0), \gamma(1)\}$.

If $S=\mathbf{R} \times \mathbf{T}^{1}$ or $\mathbf{T}^{2}$, a set $K \subset S$ is called essential if it is not contained in a topological open disc. If $K \subset S$ is not essential, we say that $K$ is inessential. If $K \subset \mathbf{T}^{2}$, a set is called fully essential if $K$ contains the complement of some disjoint union of topological open discs. If $K \subset S$ is compact and essential, we say that $K$ is vertical if $K$ is contained in a topological open annulus homotopic to the vertical annulus $\{(x, y): 0<x<1 / 2\}$. Also, a set $K \subset S$ is called annular if it is a nested intersection of compact annuli $A_{i}$ such that the inclusion $A_{i+1} \hookrightarrow A_{i}$ is a homotopy equivalence. A circloid in an annular set which does not conain any proper annular subset. Let $K \subset S$ be a compact, connected, essential and vertical set, and let $\widehat{K} \subset \mathbf{R}^{2}$ be a connected component of the preimage of $K$ by the (canonical) covering map $\mathbf{R}^{2} \rightarrow S$. As $K$ is vertical, the set $\mathbf{R}^{2} \backslash \widehat{K}$ has exactly one connected component $U_{r}$ which is unbounded to the right, and exactly one $U_{l}$ which is unbounded to the left. We denote

$$
R(\widehat{K})=U_{r} \quad L(\widehat{K})=U_{l} .
$$

A line $\ell$ is a proper embedding $\ell: \mathbf{R} \rightarrow \mathbf{R}^{2}$. Given a line $\ell$, by Shoenflies' Theorem (Cai51), there exists an orientation preserving homeomorphism $h$ of $\mathbf{R}^{2}$ such that $h \circ \ell(t)=(0, t)$, for all $t \in \mathbf{R}$. Then, the open half-plane $h^{-1}((0, \infty) \times \mathbf{R})$ is independent of $h$, and we call it the right of $\ell$, and denote it by $R(\ell)$. Analogously, we define $L(\ell)=h^{-1}((-\infty, 0) \times \mathbf{R})$ the open half-plane to the left of $\ell$. The sets $\bar{R}(\ell)$ and $\bar{L}(\ell)$ denote the closures of $R(\ell)$ and $L(\ell)$, respectively. If $\ell, \ell^{\prime}$ are two lines in $\mathbf{R}^{2}$, we define $\left(\ell, \ell^{\prime}\right)=R(\ell) \cap L\left(\ell^{\prime}\right)$, and $\left[\ell, \ell^{\prime}\right]=\bar{R}(\ell) \cap \bar{L}(\ell)$. A Brouwer curve is a line $\ell$, such that $f(\ell) \subset R(\ell)$, and a Brouwer $(0,1)$-curve $\ell$ is a Brouwer curve 
whose image by the canonical projection $\mathbf{R}^{2} \rightarrow \mathbf{R} \times \mathbf{T}^{1}$ is a simple essential loop and such that $\ell$ is oriented upwards.

Suppose that $K \subset S=\mathbf{R}^{2}, \mathbf{R} \times \mathbf{T}^{1}$ or $\mathbf{T}^{2}$ is an inessential set, and let $U \subset S$ be a topological open disc containing $K$. Let $\widehat{U} \subset \mathbf{R}^{2}$ be a lift of $U$ by the canonical covering $\pi: \mathbf{R}^{2} \rightarrow S$, and let $\widehat{K}=\pi^{-1}(K) \cap \widehat{U}$. The filling of $K$, denoted Fill $(K)$ is defined as $\pi\left(\mathbf{R}^{2} \backslash V\right)$, where $V$ is the unbounded connected component of $\mathbf{R}^{2} \backslash \widehat{K}$. A set $K \subset S$ such that $K=\operatorname{Fill}(K)$ is called a filled set.

Consider a foliation $\mathcal{F}$ with singularities of $\mathbf{R}^{2}$, and denote $\operatorname{sing}(\mathcal{F})$ the set of singularities of $\mathcal{F}$. If $\gamma \subset \mathbf{R}^{2}$ is a bounded leaf of $\mathcal{F}$, denote $\alpha(\gamma)$ and $\omega(\gamma)$ the alpha and omega-limit sets of $\gamma$, respectively. Define

$$
\operatorname{sing}(\gamma)=\operatorname{sing}(\mathcal{F}) \cap(\operatorname{Fill}(\omega(\gamma)) \cup \operatorname{Fill}(\alpha(\gamma))) .
$$

For subsets of $\mathbf{R}$ or $\mathbf{R}^{2}$ we denote the metric $d(A, B)=\sup \{|a-b|: a \in A, b \in B\}$, and when $A$ consists of a point $A=\{a\}$, we write $d(a, B)=d(A, B)$. We denote the diameter of a subset $A$ of $\mathbf{R}$ or $\mathbf{R}^{2}$ by $\operatorname{diam}(A)$, and for a subset $A \subset \mathbf{R}^{2}$ the horizontal diameter $\operatorname{diam}_{1}(A)=\operatorname{diam}\left(\operatorname{pr}_{1}(A)\right)$. Similarly, if $A \subset \mathbf{T}^{2}$, we define $\operatorname{diam}(A)=\operatorname{diam}\left(\pi^{-1}(A)\right)$ and $\operatorname{diam}_{1}(A)=\operatorname{diam}_{1}\left(\pi^{-1}(A)\right)$, where $\pi: \mathbf{R}^{2} \rightarrow \mathbf{T}^{2}$ denotes the canonical projection.

For two sets $A, B \subset \mathbf{R}^{2}$, we say that $A$ is above $B$ if $\inf \operatorname{pr}_{2}(A)>\sup _{2} \operatorname{pr}_{2}(B)$, and similarily, we say that $A$ is below $B$ if $B$ is above $A$.

\subsection{The rotation set.}

Let $f: \mathbf{T}^{2} \rightarrow \mathbf{T}^{2}$ be a homeomorphism homotopic to the identity, and let $\widehat{f}: \mathbf{R}^{2} \rightarrow \mathbf{R}^{2}$ be any lift of $f$. Consider the rotation set $\rho(\widehat{f})$ of $\widehat{f}$ as defined in the introduction. For a point $x \in \mathbf{T}^{2}$, we define its rotation set, denoted $\rho(x, \widehat{f})$, as the subset of $\mathbf{R}^{2}$ of accumulation points of sequences of the form

$$
\left(\frac{\widehat{f}^{n_{i}}(\hat{x})-\hat{x}}{n_{i}}\right)_{i \in \mathbf{N}}
$$

where $m_{i} \rightarrow \infty$ and $\hat{x} \in \pi^{-1}(x)$. When $\rho(x, \widehat{f})$ consists of a single vector $v$, we call it the rotation vector of $x$. For any subset $K \subset \mathbf{T}^{2}$, we analogously define the rotation set of $K$ by

$$
\rho(K, \widehat{f})=\cup_{x \in K} \rho(x, \widehat{f}) .
$$

If $K \subset \mathbf{R}^{2}$, we denote $\rho(K, \widehat{f})=\rho(\pi(K), \widehat{f})$.

It follows easily from the definition that

$$
\rho\left(T_{1}^{p} T_{2}^{q} \widehat{f}^{n}\right)=T_{1}^{p} T_{2}^{q}(n \rho(\widehat{f})) .
$$

Under topological conjugacies, the rotation set behaves in the following way (see for example KK08 for a proof).

Lemma 2.1. Let $f: \mathbf{T}^{2} \rightarrow \mathbf{T}^{2}$ be a homeomorphism homotopic to the identity, let $A \in \mathrm{SL}(2, \mathbf{Z})$ and let $h: \mathbf{T}^{2} \rightarrow \mathbf{T}^{2}$ be a homeomorphism isotopic to the map $\mathbf{T}^{2} \rightarrow \mathbf{T}^{2}$ induced by A. Let $\widehat{f}$ and $\widehat{h}$ be the respective lifts of $f$ and $h$. Then

$$
\rho\left(\hat{h} \hat{f}^{-1}\right)=A \rho(\widehat{f}) .
$$

In particular, $\rho\left(A \widehat{f} A^{-1}\right)=A \rho(\widehat{f})$. 
Then, if $\rho(\widehat{f})$ is a segment with rational slope, we may find $A \in \operatorname{SL}(2, \mathbf{Z})$ such that $\rho\left(A \widehat{f} A^{-1}\right)$ is vertical. Indeed, if $\rho(\widehat{f})$ has slope $p / q$, we may find integers $x, y$ such that $p x+q y=1$, and then letting

$$
A=\left(\begin{array}{cc}
p & -q \\
y & x
\end{array}\right)
$$

we have $\operatorname{det}(A)=1$, and as $A(q, p)=(0,1), A \rho(\widehat{f})$ is vertical.

By this, and using (1) and Lemma 2.1 one may easily show the following.

Lemma 2.2. Suppose that $\rho(\widehat{f})$ is a polygon with rational endpoints, and let $S$ be a side of $\rho(\widehat{f})$. Then, there exist $A \in \mathrm{SL}(2, \mathbf{Z}), m, n \in \mathbf{Z}$ and $p \in \mathbf{N}$ such that the map $G=T_{1}^{m} T_{2}^{n} A \widehat{f}^{p} A^{-1}$ satisfies:

- $\rho(G) \subset\{(x, y): x \leq 0\}$,

- $\rho(G) \cap\{(x, y): x=0\}=D$, where $D$ is the side of the polygon $\rho(G)$ given by $D=$ $T_{1}^{m} T_{2}^{n} A(p S)$.

The rotation set and periodic orbits. A rational point $\left(p_{1} / q, p_{2} / q\right) \in \rho(\widehat{f})\left(\operatorname{with} \operatorname{gcd}\left(p_{1}, p_{2}, q\right)=\right.$ 1 ) is realized by a periodic orbit if there exists a periodic point for $f$ with rotation vector $\left(p_{1} / q, p_{2} / q\right)$, or equivalently, if there is $x \in \mathbf{R}^{2}$ such that

$$
\widehat{f}^{q}(x)=x+\left(p_{1}, p_{2}\right) .
$$

We have the following realization results

Theorem 2.3 ([Fra89]). Any rational point in the interior of $\rho(\widehat{f})$ is realized by a periodic orbit.

Theorem 2.4 ([MZ89]). Every extremal point of $\rho(\widehat{f})$ is the rotation vector of some point.

The rotation set and invariant measures. For a compact $f$-invariant set $\Lambda \subset \mathbf{T}^{2}$, we denote by $\mathcal{M}_{f}(\Lambda)$ the family of $f$-invariant probability measures with support in $\Lambda$, and $\mathcal{M}_{f}=\mathcal{M}_{f}\left(\mathbf{T}^{2}\right)$. Define the displacement function $\varphi: \mathbf{T}^{2} \rightarrow \mathbf{R}^{2}$ by

$$
\varphi(x)=\widehat{f}(\widehat{x})-\widehat{x}, \quad \text { for } \widehat{x} \in \pi^{-1}(x) .
$$

This is well defined, as any two preimages of $x$ by the projection $\pi: \mathbf{R}^{2} \rightarrow \mathbf{T}^{2}$ differ by an element of $\mathbf{Z}^{2}$, and $\widehat{f}$ is $\mathbf{Z}^{2}$-periodic. Now, for $\mu \in \mathcal{M}_{f}$ we define the rotation vector of $\mu$ as

$$
\rho(\mu, \widehat{f})=\int \varphi d \mu \text {. }
$$

Then, we define the sets

$$
\rho_{\text {mes }}(\Lambda, \widehat{f})=\left\{\rho(\mu, \widehat{f}): \mu \in \mathcal{M}_{f}(\Lambda)\right\}
$$

and

$$
\rho_{\text {erg }}(\Lambda, \widehat{f})=\{\rho(\mu): \mu \text { is ergodic for } f \text { and } \operatorname{supp}(\mu) \subset \Lambda\} .
$$

When $\Lambda=\mathbf{T}^{2}$ we simply write $\rho_{\text {mes }}(\widehat{f})$ and $\rho_{\text {erg }}(\widehat{f})$.

Proposition 2.5 ([MZ89]). It holds the following:

$$
\rho(\widehat{f})=\rho_{\text {mes }}(\widehat{f})=\operatorname{conv}\left(\rho_{\text {erg }}(\widehat{f})\right) .
$$




\subsection{Atkinson's Lemma.}

Let $(X, \mu)$ be a probability space, $T: X \rightarrow X$ be an ergodic transformation with respect to $\mu$, and $\phi: X \rightarrow \mathbf{R}$ a measurable map. We say that the pair $(T, \phi)$ is recurrent if for any measurable set $A \subset X$ of positive measure, and every $\epsilon>0$ there is $n>0$ such that

$$
\mu\left(A \cap T^{-n}(A) \cap\left\{x: \sum_{i=0}^{n-1}\left|\phi\left(T^{i}(x)\right)\right|<\epsilon\right\}\right)>0 .
$$

In [Atk76] it is proved the following theorem.

Theorem 2.6. Let $(X, \mu)$ be a non-atomic probability space, $T: X \rightarrow X$ an ergodic automorphism, and $\phi: X \rightarrow \mathbf{R}$ an integrable function. Then, the pair $(T, \phi)$ is recurrent if and only if $\int \phi d \mu=0$.

From this theorem, it is not difficult to obtain the following corollary, usually known as 'Atkinson's Lemma'.

Corollary 2.7. Let $X$ be a separable metric space and $\mu$ a probability measure in $X$ which is ergodic with respect to a measurable transformation $T: X \rightarrow X$. Let $\phi: X \rightarrow \mathbf{R}$ be an integrable function, with $\int \phi d \mu=0$. Then, there exists a full $\mu$-measure set $\widetilde{X} \subset X$ such that for any $x \in \tilde{X}$, there is a sequence of positive integers $n_{i}$ with

$$
T^{n_{i}}(x) \rightarrow x \text { and }\left|\sum_{j=0}^{n_{i}-1} \phi\left(T^{j}(x)\right)\right| \rightarrow 0 \quad \text { as } i \rightarrow \infty .
$$

\subsection{A theorem of Handel}

Denote by $\mathbf{A}$ the compact annulus $\mathbf{A}=[0,1] \times \mathbf{T}^{1}$, and by $\widetilde{\mathbf{A}}$ its universal cover $\widetilde{\mathbf{A}}=[0,1] \times \mathbf{R}$. Let $h: \underset{\widetilde{A}}{\rightarrow} \mathbf{A}$ be an orientation preserving, boundary component preserving homeomorphism, and let $\widetilde{h}: \widetilde{\mathbf{A}} \rightarrow \widetilde{\mathbf{A}}$ be a lift of $h$.

For a point $x \in \mathbf{A}$, and a lift $\tilde{x} \in \tilde{\mathbf{A}}$ of $x$, the limit

$$
\rho(x, \widetilde{h})=\lim _{n \rightarrow \infty} \frac{\operatorname{pr}_{2}\left(\widetilde{h}^{n}(\widetilde{x})-\widetilde{x}\right)}{n},
$$

whenever it exists is independent of the lift $\tilde{x}$, and it is called the rotation number of $x$. The pointwise rotation set of $\widehat{h}$ is defined as

$$
\rho_{\text {point }}(\widetilde{h})=\bigcup \rho(x, \widetilde{h})
$$

where the union is taken over all the $x$ in the domain of $\rho(\cdot, \widetilde{h})$.

The following theorem is part of Theorem 0.1 in Han90.

Theorem 2.8. If $h:[0,1] \times \mathbf{T}^{1} \rightarrow[0,1] \times \mathbf{T}^{1}$ is an orientation preserving, boundary component preserving homeomorphism and $\tilde{f}:[0,1] \times \mathbf{R} \rightarrow[0,1] \times \mathbf{R}$ is any lift, then:

1. $\rho_{\text {point }}(\tilde{f})$ is a closed set.

2. With the exception of at most a discrete set of values $r$ in $\rho_{\text {point }}(\widetilde{h})$, there is a compact invariant set $Q_{r}$ such that $\rho(x, \widetilde{h})=r$ for all $x \in Q_{r}$. If $r$ is rational, then $Q_{r}$ exists and is realized by a periodic orbit. 


\subsection{Stable and unstable sets, in the case there is a Brouwer curve.}

Let $\widehat{f}$ be any lift of a homeomorphism of $\mathbf{T}^{2}$ homotopic to the identity. Suppose there is a Brouwer $(0,1)$-curve $\ell$ for $\widehat{f}$. For $i \in \mathbf{N}_{0}$, denote $\ell_{i}=T_{1}^{i}(\ell)$.

Following [Dáv13], for each $i \in \mathbf{N}$, we define the sets $L_{\infty}^{i}$ and $R_{\infty}^{i}$, which in some sense are the 'stable' and 'unstable' sets, respectively, of the maximal invariant set in $\left[\ell_{i}, \ell_{i+1}\right]$ for $\widehat{f}$. Let

$$
R_{\infty}^{i}=\bigcap_{n \in \mathbf{Z}} R\left(\widehat{f}^{n}\left(\ell_{i}\right)\right), \quad \text { and } \quad L_{\infty}^{i}=\bigcap_{n \in \mathbf{Z}} L\left(\widehat{f}^{-n}\left(\ell_{i+1}\right)\right)
$$

(see Fig. 2)

By definition, the sets $R_{\infty}^{i}$, and $L_{\infty}^{i}$ are $\widehat{f}$-invariant. As $\ell$ is a Brouwer curve for $\widehat{f}, \widehat{f}\left(\ell_{i}\right) \subset$ $R\left(\ell_{i}\right)$ for all $i$. Therefore,

$$
R_{\infty}^{i}=\left\{x \in \mathbf{R}^{2}: \widehat{f}^{-n}(x) \in R\left(\ell_{i}\right) \forall n \geq 0\right\},
$$

and

$$
L_{\infty}^{i}=\left\{x \in \mathbf{R}^{2}: \widehat{f}^{n}(x) \in L\left(\ell_{i+1}\right) \forall n \geq 0\right\},
$$

Therefore, for each $i$, the set $R_{\infty}^{i} \cap L_{\infty}^{i}$ is the maximal invariant set of $\left[\ell_{i}, \ell_{i+1}\right]$ for $\widehat{f}$. Observe that, either the set $R_{\infty}^{i} \cap L_{\infty}^{i}$ is non-empty for all $i$, or $\widehat{f}^{n}\left(\ell_{0}\right) \subset R\left(\ell_{1}\right)=R\left(T_{1}\left(\ell_{0}\right)\right)$ for some $n$.

We have

$$
R_{\infty}^{i} \cap\left[\ell_{i}, \ell_{i+1}\right]=\left\{x \in\left[\ell_{i}, \ell_{i+1}\right]: d\left(\widehat{f}^{-n}(x), L_{\infty}^{i} \cap R_{\infty}^{i}\right) \rightarrow 0 \text { as } n \rightarrow \infty\right\},
$$

and

$$
L_{\infty}^{i} \cap\left[\ell_{i}, \ell_{i+1}\right]=\left\{x \in\left[\ell_{i}, \ell_{i+1}\right]: d\left(\widehat{f}^{n}(x), L_{\infty}^{i} \cap R_{\infty}^{i}\right) \rightarrow 0 \text { as } n \rightarrow \infty\right\} .
$$

That is, the set $L_{\infty}^{i} \cap\left[\ell_{i}, \ell_{i+1}\right]$ can be thought as the 'local stable set' of $R_{\infty}^{i} \cap L_{\infty}^{i}$, and $R_{\infty}^{i} \cap$ $\left[\ell_{i}, \ell_{i+1}\right]$ can be thought as the 'local unstable set' of $R_{\infty}^{i} \cap L_{\infty}^{i}$.
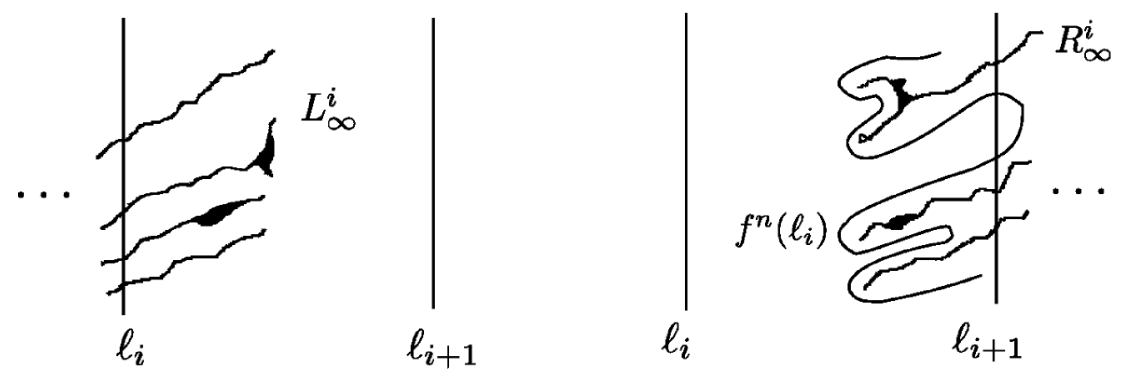

Figure 2: Some examples of the sets $L_{\infty}^{i}$ and $R_{\infty}^{i}$.

The following lemma is proved in Dáv13] (Lemma 6.8 in that article).

Lemma 2.9. For every $i \geq 0$ :

1. if $C$ is a connected component of $R_{\infty}^{i}$, then $\sup \operatorname{pr}_{1}(C)=+\infty$, and

2. if $C^{\prime}$ is a connected component of $L_{\infty}^{i}$, then $\inf \operatorname{pr}_{1}\left(C^{\prime}\right)=-\infty$.

The following lemma is a consequence of the periodicity of $\widehat{f}$. For a proof, see Lemma 6.10 in Dáv13. 
Lemma 2.10. Let $i \in \mathbf{N}$ and suppose that there is $n>0$ such that $\widehat{f}^{n}(\ell) \cap R\left(\ell_{i}\right) \neq \emptyset$. There is $K>0$ such that if $C \subset\left(\ell, \ell_{i}\right)$ is forward invariant, then $\operatorname{diam}_{2}(C)<K$.

As a consequence of the last two lemmas we have the following.

Lemma 2.11. Let $i \in \mathbf{N}$ and suppose that there is $n>0$ such that $\widehat{f}^{n}(\ell) \cap R\left(\ell_{i}\right) \neq \emptyset$. If $C$ is a connected component of $L_{\infty}^{i} \cap R(\ell)$, we have:

- $\bar{C}$ is compact,

- $\rho(\bar{C}, \widehat{f})$ consists of a point,

- $\bar{C} \cap \ell \neq \emptyset$.

Proof. To prove that $\bar{C}$ is compact, first note that $\bar{C} \subset L_{\infty}^{1} \cap \bar{R}(\ell), \bar{C}$ is forward invariant, and $\hat{f}^{n}(\bar{C}) \subset L\left(\ell_{2}\right)$ for all $n$. If $\bar{C}$ was not compact, it would be unbounded in the vertical direction, and this would contradict Lemma 2.10 .

If $\rho(C, \widehat{f})$ consisted of more than one point, then its forward iterates would be contained in $\left(\ell, \ell_{2}\right)$ and would have arbitrarily large vertical diameter, which would also contradict Lemma 2.10

Finally, the fact that $\bar{C} \cap \ell \neq \emptyset$ is a consequence of Lemma 2.9

\subsection{Brouwer Theory}

\subsubsection{Brouwer foliations.}

Let $\mathcal{I}=\left(f_{t}\right)_{t \in[0,1]}$ be an isotopy on a surface $S$ from $f_{0}=$ Id to a homeomorphism $f_{1}=f$. We say that a point $x \in S$ is contractible for $f$ if the isotopy loop $f_{t}(x)$ is homotopically trivial in $S$.

Given a topological oriented foliation $\mathcal{F}$ of $S$, we say that the isotopy $\mathcal{I}$ is tranverse to $\mathcal{F}$ if for each $x \in S$, the isotopy path $f_{t}(x)$ is homotopic with fixed endpoints to an arc which is positively transverse to $\mathcal{F}$ in the usual sense.

A clear obstruction to the existence of a foliation transverse to $\mathcal{I}$ is the existence of contractible points for $f$. The following theorem from Le Calvez tells us that this is the only obstruction.

Theorem 2.12 (Cal05]). If there are no contractible fixed points for $f$, then there exists a foliation $\mathcal{F}$ without singularities which is transverse to $\mathcal{I}$.

In the case that there exist contractible fixed points for $f$, the previous theorem is still useful. This is thanks to the following result from Jaulent.

Theorem 2.13 ( Jau13 $)$. Given an isotopy $\mathcal{I}=\left(f_{t}\right)_{t \in[0,1]}$ on $S$ from the identity to a homeomorphism $f$, there exists a closed set $X \subset \operatorname{Fix}(f)$ and an isotopy $\mathcal{I}^{\prime}=\left(f_{t}^{\prime}\right)_{t \in[0,1]}$ on $S \backslash X$ from $\operatorname{Id}_{S \backslash X}$ to $\left.f\right|_{S \backslash X}$ such that

- for each $z \in S \backslash X$ the arc $\left(f_{t}^{\prime}(z)\right)_{t \in[0,1]}$ is homotopic with fixed endpoints (in $S$ ) to $\left(f_{t}(z)\right)_{t \in[0,1]}$,

- there are no contractible fixed points for $\left.f\right|_{S \backslash X}$ with respect to $\mathcal{I}^{\prime}$.

Remark 2.14. By Theorem 2.12 it follows that there exists a foliation $\mathcal{F}_{X}$ of $S \backslash X$ without singularities which is transverse to $\mathcal{I}^{\prime}$. 
Remark 2.15. If the set $X$ is totally disconnected, the isotopy $\mathcal{I}^{\prime}$ may be extended to an isotopy on $S$ that fixes $X$; that is, $f_{t}^{\prime}(x)=x$ for each $x \in X$ and all $t \in[0,1]$. Similarly, the foliation $\mathcal{F}_{X}$ may be extended to a foliation with singularities $\mathcal{F}$ on $S$, where the set of singularities of $\mathcal{F}$ coincides with $X$. Also, if $\widehat{\mathcal{I}}^{\prime}=\left(\widehat{f}_{t}^{\prime}\right)_{t \in[0,1]}$ and $\widehat{\mathcal{I}}=\left(\widehat{f}_{t}\right)_{t \in[0,1]}$ are the respective canonical lifts of $\mathcal{I}^{\prime}$ and $\mathcal{I}$ to the universal cover of $S$, then $\widehat{f}_{1}^{\prime}=\widehat{f}_{1}$. This follows from the fact that, if $z \in S \backslash X$, the paths $\left(f_{t}^{\prime}(z)\right)_{t}$ and $\left(f_{t}(z)\right)_{t}$ are homotopic with fixed points in $S$.

\subsubsection{Fixing the isotopy $\left(f_{t}\right)_{t}$, the foliation $\mathcal{F}$ transversal to $\left(f_{t}\right)_{t}$, the set $X \subset \operatorname{Fix}(f)$ and their lifts $\left(\widehat{f}_{t}\right)_{t}, \widehat{\mathcal{F}}, \widehat{X}$ to $\mathbf{R}^{2}$.}

Let $f$ and $\widehat{f}$ be as in Theorem D. From now on we fix an isotopy $\left(f_{t}\right)_{t}$ on $\mathbf{T}^{2}$ from $f_{0}=$ Id to $f_{1}=f$ such that the canonical lift $\left(\widehat{f}_{t}\right)_{t}$ of $\left(f_{t}\right)_{t}$ to $\mathbf{R}^{2}$ ends in $\widehat{f}_{1}=\widehat{f}$. By Theorems 2.12 and 2.13 and by Remark 2.14, there exists a set $X \subset \operatorname{Fix}(f)$, an isotopy $\left(f_{t}\right)_{t}$ on $\mathbf{T}^{2} \backslash X$ from Id to $f$ and a foliation $\mathcal{F}_{X}$ of $\mathbf{T}^{2} \backslash X$ such that $\left(f_{t}\right)_{t}$ is transverse to $\mathcal{F}_{X}$. Let $\widehat{\mathcal{F}}$ and $\widehat{X}$ be lifts of $\mathcal{F}$ and $X$ to $\mathbf{R}^{2}$, respectively.

Note that if $X$ is totally disconnected, then by Remark 2.15 we have that:

- $\left(f_{t}\right)_{t}$ extends to an isotopy on $\mathbf{T}^{2}$, denoted also by $\left(f_{t}\right)_{t}$, that fixes $X$,

- there exists a foliation $\mathcal{F}$ of $\mathbf{T}^{2}$ with $\operatorname{sing}(\mathcal{F})=X$ and such that $\mathcal{F} \backslash \operatorname{sing}(\mathcal{F})$ is transverse to the isotopy $\left(f_{t}\right)_{t}$ restricted to $\mathbf{T}^{2} \backslash X$.

Remark 2.16. Suppose that the set $X$ is totally disconnected. Consider a closed set $F \subset \widehat{X}$ and the universal covering $\mathbf{D} \rightarrow \mathbf{R}^{2} \backslash F$. Whenever we refer to the canonical lift of $\widehat{f}$ to $\mathbf{D}$, it will be implicit that it is the canonical lift with respect to the isotopy $\left(f_{t}\right)_{t}$.

\subsubsection{Some lemmas.}

Through all of this section we suppose that the set $X$ from $\$ 2.6 .2$ is totally disconnected. The following lemma is trivial.

Lemma 2.17. Suppose that a leaf $\ell$ of $\widehat{\mathcal{F}}$ is a Brouwer curve for $\widehat{f}$. Let $\beta$ be a compact arc such that $\beta(1) \in \gamma$. We have:

1. if $\beta(0) \in \widehat{X} \cap L(\ell)$ then $\widehat{f}^{n}(\beta) \cap \ell \neq \emptyset$ for all $n \in \mathbf{N}$.

2. if $\beta(0) \in \widehat{X} \cap R(\ell)$ then $\widehat{f}^{-n}(\beta) \cap \ell \neq \emptyset$ for all $n \in \mathbf{N}$.

Recall that if $\gamma \in \widehat{\mathcal{F}}, \operatorname{sing}(\gamma)$ denotes the set of singularities of $\widehat{\mathcal{F}}$ contained in $\operatorname{Fill}(\omega(\gamma)) \cup$ $\operatorname{Fill}(\alpha(\gamma))$.

Definition 2.18. Let $\gamma \subset \mathbf{R}^{2}$ be a leaf of $\widehat{\mathcal{F}}$, and let $\beta:[0,1] \rightarrow \mathbf{R}^{2}$ be such that $\beta\left(t_{0}\right) \in \gamma$, for some $t_{0} \in[0,1]$. Consider a lift $\bar{\gamma}$ of $\gamma$ to the universal cover of $\mathbf{R}^{2} \backslash \operatorname{sing}(\gamma)$, and let $\bar{\beta}$ be the lift of $\beta$ such that $\bar{\beta}\left(t_{0}\right) \in \bar{\gamma}$.

1. We say that $\beta$ arrives in $\gamma$ in $t_{0}$ by the left if $t_{0}>0$ and if there is $t_{1} \in\left(0, t_{0}\right)$ such that $\bar{\beta}(t) \in L(\bar{\gamma})$ for all $t \in\left(t_{1}, t_{0}\right)$. Similarily, we say that $\beta$ arrives in $\gamma$ in $t_{0}$ by the right if $t_{0}>0$ and there is $t_{1} \in\left(0, t_{0}\right)$ such that $\bar{\beta}(t) \in R(\bar{\gamma})$ for all $t \in\left(t_{1}, t_{0}\right)$.

2. We say that $\beta$ leaves $\gamma$ in $t_{0}$ by the right if $t_{0}<1$ and if $\beta^{-1}$ arrives in $\gamma$ in $t_{0}$ by the right. Also, we say that $\beta$ leaves $\gamma$ in $t_{0}$ by the left if $t_{0}<1$ and $\beta^{-1}$ arrives in $\gamma$ in $t_{0}$ by the left. 
Next lemma describes how an arc might get 'anchored' to a leaf of $\widehat{\mathcal{F}}$. For a leaf $\gamma \in \widehat{\mathcal{F}}$ recall our notation $\operatorname{sing}(\gamma)=\operatorname{sing}(\widehat{\mathcal{F}}) \cap(\operatorname{Fill}(\alpha(\gamma)) \cup \operatorname{Fill}(\omega(\gamma)))$.

Lemma 2.19. Let $\gamma$ be a non-compact leaf of $\widehat{\mathcal{F}}$ with compact closure, and let $S=\operatorname{sing}(\gamma)$ (see Fig. 3). Suppose that $\beta \subset \mathbf{R}^{2} \backslash S$ is an arc such that $\beta(0) \in \widehat{X} \backslash S, \beta(1) \in \gamma$, and $\beta$ is homotopic wfe $\operatorname{Rel}(S)$ to an arc $\beta_{1}$ such that the only intersection of $\beta_{1}$ with $\gamma$ is the point $\beta_{1}(1)$.

1. If $\beta_{1}$ arrives in $\gamma$ in 1 by the left, then $\widehat{f}^{n} \beta \cap \gamma \neq \emptyset$ for all $n \in \mathbf{N}$.

2. If $\beta_{1}$ arrives in $\gamma$ in 1 by the right, then $\widehat{f}^{-n} \beta \cap \gamma \neq \emptyset$ for all $n \in \mathbf{N}$.

Proof. We will prove item 1, item 2 being analogous. By the Poincaré-Bendixson Theorem we know that $S$ is non-empty. Consider the universal covering space of $\mathbf{R}^{2} \backslash S$, which is homeomorphic to the unit disc $\mathbf{D}$, and the canonical lift $\bar{f}: \mathbf{D} \rightarrow \mathbf{D}$ of $\left.\widehat{f}\right|_{\mathbf{R}^{2} \backslash S}$. Any lift of $\gamma$ to $\mathbf{D}$ is a Brouwer curve for $\bar{f}$.

Consider lifts $\bar{\beta}, \bar{\beta}_{1}$ and $\bar{\gamma}$ of the arcs $\beta, \beta_{1}$ and the leaf $\gamma$, respectively, being these lifts such that $\bar{\beta}(1)=\bar{\beta}_{1}(1) \in \bar{\gamma}$. As $\beta$ is homotopic wfe $\operatorname{Rel}(S)$ to $\beta_{1}$, we have that $\bar{\beta}(0)=\bar{\beta}_{1}(0)$. The arc $\bar{\beta}_{1}$ intersects $\bar{\gamma}$ only in $\bar{\beta}_{1}(1)$ and arrives in $\gamma$ in 1 by the left, and therefore $\bar{\beta}(0)=\bar{\beta}_{1}(0) \in L(\bar{\gamma})$. By Lemma 2.17 we have that $\bar{f}^{n}(\bar{\beta})$ intersects $\bar{\gamma}$ for all $n \in \mathbf{N}$, and therefore $\widehat{f}^{n} \beta$ intersects $\gamma$ for all $n$.

Remark 2.20. In last proof we see that, for all $n \in \mathbf{N},\left.\widehat{f}^{n} \beta\right|_{[0,1)} \cap \neq \emptyset$.

For $t \in \mathbf{R}$, recall our notation

$$
\widehat{f_{t}}:=\widehat{f}_{t} \bmod 1 \circ \widehat{f}^{\lfloor t\rfloor}
$$

(cf. Section 2.1), and note that $\widehat{f}_{n}=\widehat{f}^{n}$ for $n \in \mathbf{Z}$.

Lemma 2.21. Let $F \subset \mathbf{R}^{2}$ be a closed subset, and let $N \in \mathbf{N}$. Let $\gamma, \gamma_{0}:[0, N] \rightarrow \mathbf{R}^{2}$ be arcs disjoint from $F$, and such that $\gamma$ and $\gamma_{0}$ are homotopic wfe $\operatorname{Rel}(F)$ (see Fig. 3). Let $\delta$ be the $\operatorname{arc}\left(\widehat{f}_{t}(\gamma(1))\right)_{t \in[0, N]}$. Then, if $\left\{\widehat{f}_{t}(F): t \in[0, N]\right\}$ is disjoint from $\gamma_{0} \cdot \delta$, the arc $\widehat{f}_{N}(\gamma)$ and the curve $\gamma_{0} \cdot \delta$ are homotopic wfe $\operatorname{Rel}\left(\widehat{f}^{N}(F)\right)$.

Proof. For $t \in[0, N]$ define $\delta_{t}:=\left.\delta\right|_{[0, t]}$. For each $0 \leq t \leq N$, the $\operatorname{arc} \Gamma_{t}:=\widehat{f}_{t}(\gamma) \cdot \delta_{t}^{-1} \cdot \gamma_{0}^{-1}$ is a (non necessarily simple) loop. As $\gamma$ and $\gamma_{0}$ are disjoint from $F$, and homotopic with fixed endpoints and $\operatorname{Rel}(F)$, we have that $F$ is contained in the unbounded component of $\mathbf{R}^{2} \backslash \Gamma_{0}$. By continuity, and as by hypothesis $\left\{\widehat{f}_{t}(F): t \in[0, N]\right\}$ is disjoint from $\gamma_{0} \cdot \delta=\gamma_{0} \cdot \delta_{N}$, we have that for any $t, \widehat{f}_{t}(F)$ is contained in the unbounded component of $\mathbf{R}^{2} \backslash \Gamma_{t}$, and in particular for $t=N$. This implies in turn that $\Gamma_{N}$ is homotopically trivial $\operatorname{Rel}\left(\widehat{f}^{N}(F)\right)$, or equivalently, the $\operatorname{arcs} \widehat{f}^{N}(\gamma)$ and $\gamma_{0} \cdot \delta_{N}=\gamma_{0} \cdot \delta$ are homotopic wfe $\operatorname{Rel}\left(\widehat{f}^{N}(F)\right)$.

\subsection{Poincaré-Bendixson theory.}

This section follows $[\mathrm{KT}]$. Suppose that the set $X=\operatorname{sing}(\mathcal{F})$ is totally disconnected. We call a leaf of $\mathcal{F}$ a connection if both its $\alpha$-limit and its $\omega$-limit are sets consisting of one element of $\operatorname{sing}(\mathcal{F})$. Define a generalized cycle of connections of $\mathcal{F}$ as a loop $\Gamma$ such that $\Gamma \backslash \operatorname{sing}(\mathcal{F})$ is a (not necessarily finite) disjoint union of regular leaves of $\mathcal{F}$, with their orientation coinciding with that of $\Gamma$.

The following is a generalization of the Poincaré-Bendixson Theorem, and is a particular case of a theorem of Solntzev [Sol45] (cf. also [NS89] §1.78) and can be stated in terms of continuous flows due to a theorem of Gutiérrez Gut79]. 

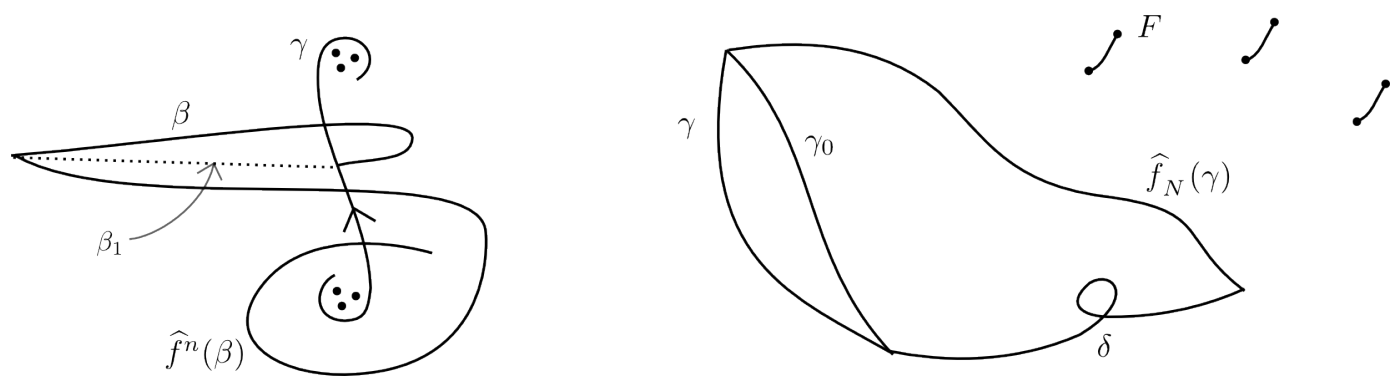

Figure 3: Lemma 2.19 (left), and Lemma 2.21 (right).

Theorem 2.22. Let $\phi=\left\{\phi_{t}\right\}_{t \in \mathbf{R}}$ be a continuous flow on $\mathbf{R}^{2}$ with a totally disconnected set of singularities. If the forward orbit of a point $\left\{\phi_{t}(z)\right\}_{t \geq 0}$ is bounded, then its $\omega$-limit is one of the following:

- a singularity;

- a closed orbit;

- a generalized cycle of connections.

As $\mathcal{F}$ is an oriented foliation with singularities, it can be embeded in a flow (cf. Whi33. Whi41]) and we may apply last theorem to $\mathcal{F}$.

\subsection{Intersection number of certain lines.}

Let $\Gamma_{1}, \Gamma_{2} \subset \mathbf{R}^{2}$ be two lines. We say that $\Gamma_{1}$ and $\Gamma_{2}$ have disjoint ends if there is $T>0$ such that

$$
\inf _{t>T} d\left(\left\{\Gamma_{1}(t), \Gamma_{1}(-t)\right\},\left\{\Gamma_{2}(t), \Gamma_{2}(-t)\right\}\right)>0 .
$$

If $\Gamma_{1}$ and $\Gamma_{2}$ have disjoint ends, then the algebraic intersection number of $\Gamma_{1}$ and $\Gamma_{2}$, denoted $\operatorname{IN}\left(\Gamma_{1}, \Gamma_{2}\right)$ is well defined (although it is not invariant under arbitrary isotopies). We use the usual convention that $\operatorname{IN}\left(\Gamma_{1}, \Gamma_{2}\right)=+1$ if $\Gamma_{1}$ traverses $\Gamma_{2}$ from the left to the right. We will make use of the following basic fact.

Lemma 2.23. Suppose that $\Gamma_{1}$ and $\Gamma_{2}$ are lines with disjoint ends, and $\operatorname{IN}\left(\Gamma_{1}, \Gamma_{2}\right)=1$. If $t_{1}, t_{2}$ are such that

$$
\begin{aligned}
& t_{1}<\min \left\{t \in \mathbf{R}: \Gamma_{1}(t) \in \Gamma_{2}\right\}, \\
& t_{2}>\max \left\{t \in \mathbf{T}: \Gamma_{1}(t) \in \Gamma_{2}\right\},
\end{aligned}
$$

then $\Gamma_{1}\left(t_{1}\right) \in L\left(\Gamma_{2}\right)$ and $\Gamma_{1}\left(t_{2}\right) \in R\left(\Gamma_{2}\right)$.

\section{Theorems A, B and C.}

Theorem B. This theorem is an immediate consequence of Theorem $\mathrm{D}$ if the vector $v \in \mathbf{Q}^{2}$ from the statement is vertical. The general case $v \in \mathbf{Q}^{2} \backslash\{(0,0)\}$, is reduced to the case of vertical $v$ by standard arguments, using (1) and Lemma 2.1 
Theorem A. Also, if $\rho(\widehat{f})$ is a vertical segment of the form $\{0\} \times I$, Theorem A follows immediately from Theorem $\mathrm{D}$, and the case of a general rational interval is reduced to this case by the use of (1) and Lemma 2.1 .

Theorem C. This theorem is an easy consequence of Theorem B. Let $S$ be a side of the polygon $\rho(\widehat{f})$. Recall that by Lemma 2.2 there exist $A \in \mathrm{SL}(2, \mathbf{Z}), m, n \in \mathbf{Z}$ and $p \in \mathbf{N}$ such that the map $G=T_{1}^{m} T_{2}^{n} A \widehat{f}^{p} A^{-1}$ satisfies

- $\rho(G) \subset\{(x, y): x \leq 0\}$,

- $\rho(G) \cap\{(x, y): x=0\}=D$, where $D$ is the side of the polygon $\rho(G)$ given by $D=$ $T_{1}^{m} T_{2}^{n} A(p S)$.

Let $w \in \mathbf{Q}^{2}$ be the unit vector orthogonal to $S$ pointing outwards $\rho(\widehat{f})$. From the definition of the rotation set, (1) and Lemma 2.1 one may also show the following:

- there is $m_{1}>0$ such that

$$
\sup \left\{\operatorname{pr}_{1}\left(G^{n}(x)-x\right): x \in \mathbf{R}^{2}, n \in \mathbf{N}\right\}<m_{1}
$$

if and only if there is $m_{2}>0$ such that

$$
\sup \left\{\left\langle\widehat{f}^{n}(x)-x-n r, w\right\rangle: x \in \mathbf{R}^{2}, r \in \rho(\widehat{f}), n \in \mathbf{N}\right\}<m_{2} .
$$

Observe that, as $\rho(G) \subset\{(x, y): x \leq 0\}$, by Theorem B there is $m_{1}>0$ such that $(2)$ holds. In this way, we have that for any side $S_{i}$ of $\rho(\widehat{f})$, if $w_{i}$ is the unit vector orthogonal to $S_{i}$ pointing outwards $\rho(\widehat{f})$, there is $m_{1}^{i}>0$ such that

$$
\sup \left\{\left\langle\widehat{f}^{n}(x)-x-n r, w_{i}\right\rangle: x \in \mathbf{R}^{2}, r \in \rho(\widehat{f}), n \in \mathbf{N}\right\}<m_{1}^{i} .
$$

Letting $m=2 \max \left\{m_{1}^{i}\right\}$, it follows that

$$
\sup \left\{d\left(\widehat{f}^{n}(x)-x, n \rho(\widehat{f})\right): x \in \mathbf{R}^{2}, n \in \mathbf{N}\right\}<m,
$$

as desired.

\section{Proof of Theorem D.}

The organization of the proof of Theorem $\mathrm{D}$ is as follows. Recall that the isotopy $\left(f_{t}\right)_{t}$ from Id to $f$ and the foliation $\mathcal{F}$ with set of singularities $X \subset \operatorname{Fix}(f)$ were fixed in $\$ 2.6 .2$ In Section 4.1 we study mainly under what conditions one may assume that $\operatorname{sing}(\widehat{\mathcal{F}})$ is inessential. In Section 4.2 we show there exists an essential circloid $\mathcal{C} \subset \mathbf{R} \times \mathbf{T}^{1}$ which is formed by leaves and singularities of the foliation $\widetilde{\mathcal{F}}$, which is the lift of $\mathcal{F}$ to $\mathbf{R} \times \mathbf{T}^{1}$. Using such circloid, in Section 4.3 we construct the bound $M(f)>0$ which will be used to show that, if there are horizontal displacements larger than $M(f)$, then we may assume that $\operatorname{sing}(\widehat{\mathcal{F}})$ is totally disconnected (Section 4.4) and there is linear horizontal speed (Sections 4.5 and 4.6). 


\subsection{About inessential sets and the set $\operatorname{sing}(\mathcal{F})$.}

We begin with the following.

Lemma 4.1. There exist essential vertical loops $c_{1}$ and $c_{2}$ in $\mathbf{T}^{2}$ which are positively transverse to $\mathcal{F}$, disjoint from $\operatorname{sing}(\mathcal{F}), c_{1}$ is oriented upwards and $c_{2}$ is oriented downwards.

Proof. By the hypotheses of Theorem D, $\rho(\widehat{f})$ contains points $(0, a)$ and $(0, b)$, with $a<0<$ $b$. If $\max _{1}(\rho(\widehat{f}))>0$, then by Theorem 2.3 there is a periodic point $p \in \mathbf{T}^{2}$ such that $\operatorname{pr}_{1}(\rho(p, \widehat{f}))>0$, and then Theorem D holds. Therefore, we may assume there exist extremal points of $\rho(\widehat{f})$ of the form $\left(0, e_{1}\right),\left(0, e_{2}\right)$, with $e_{1}<0<e_{2}$, and by Proposition 2.5 there are ergodic measures $\mu_{1}, \mu_{2}$ for $f$, with $\rho\left(\mu_{1}, \widehat{f}\right)=\left(0, e_{1}\right)$ and $\rho\left(\mu_{2}, \widehat{f}\right)=\left(0, e_{2}\right)$.

Consider the displacement function $\varphi: \mathbf{T}^{2} \rightarrow \mathbf{R}^{2}, \varphi(x)=\widehat{f}(\widehat{x})-\widehat{x}$, where $\widehat{x} \in \pi^{-1}(x)$ and $\pi: \mathbf{R}^{2} \rightarrow \mathbf{T}^{2}$ denotes the canonical projection. Let $\varphi_{1}=\operatorname{pr}_{1} \circ \varphi$. As $\int \varphi_{1} d \mu_{2}=0$, by Atkinson's Lemma 2.7 there exists a $\mu_{2}$-total measure set $X_{1} \subset \mathbf{T}^{2}$ such that, for any $x \in X_{1}$, there is a sequence of integers $n_{i}$ such that

$$
f^{n_{i}}(x) \rightarrow x \quad \text { and } \quad \sum_{j=0}^{n_{i}-1} \varphi_{1}\left(f^{n_{i}}(x)\right) \rightarrow 0 \quad \text { as } i \rightarrow \infty .
$$

By Birkhoff's Theorem there exists a $\mu_{2}$-total measure set $X_{2} \subset \mathbf{T}^{2}$ such that, for any $x \in X_{2}$,

$$
\frac{1}{n_{i}} \sum_{j=0}^{n_{i}-1} \varphi\left(f^{n_{i}}(x)\right)=\frac{\widehat{f}^{n_{i}}(\widehat{x})-\widehat{x}}{n_{i}} \longrightarrow \int \varphi d \mu_{2}=\left(0, e_{2}\right) \quad \text { as } i \rightarrow \infty,
$$

where $\widehat{x} \in \pi^{-1}(x)$.

Let $x \in X_{1} \cap X_{2}$ and $\widehat{x} \in \pi^{-1}(x)$. Consider a flow box $B$ for the foliation $\mathcal{F}$ containing $x$, and let $\widehat{B}$ be a lift of $B$ containing $\widehat{x}$. By $(3)$ and $(4)$ there exist integers $i, m \in \mathbf{N}$ such that $\widehat{f}^{n_{i}}(\widehat{x}) \in T_{2}^{m}(B)$. As the isotopy $\widehat{f}_{t}$ is transverse to $\widehat{\mathcal{F}}$, there exists an arc $\widehat{\gamma}$ going from $\widehat{x}$ to $\widehat{f}^{n_{i}}(\widehat{x})$, positively transverse to $\widehat{\mathcal{F}}$ (and also homotopic wfe $\operatorname{Rel}(\operatorname{sing}(\widehat{\mathcal{F}}))$ to the isotopy arc $\left.\left(\widehat{f}_{t}\right)_{t \in\left[0, n_{i}\right]}\right)$.

Let $\gamma=\pi(\widehat{\gamma}) \subset \mathbf{T}^{2}$. The point $f^{n_{i}}(x) \in B$ might be joined to a point $p \in \gamma \cap B$ by an arc $\gamma^{\prime} \subset B$ positively transverse to $\mathcal{F}$ (see Fig. 4). If $\gamma_{1}$ is the subarc of $\gamma$ from $p$ to $f^{n_{i}}(x)$, then the loop $c_{1}=\gamma_{1} \cdot \gamma^{\prime}$ is vertical, positively transverse to $\mathcal{F}$, disjoint from $\operatorname{sing}(\mathcal{F})$ and oriented upwards.

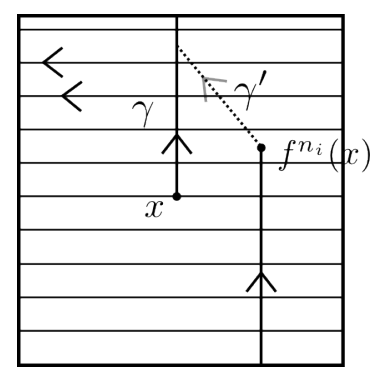

Figure 4:

In a symmetric way, using the ergodic measure $\mu_{1}$, one constructs a vertical loop $c_{2} \subset \mathbf{T}^{2}$ which is oriented downwards, positively transverse to $\mathcal{F}$ and disjoint from $\operatorname{sing}(\mathcal{F})$. 
Lemma 4.2. Let $c \subset \mathbf{T}^{2} \backslash \operatorname{sing}(\mathcal{F})$ be any essential vertical loop (which exists by Lemma 4.1). Then, if

$$
\sup \left\{\operatorname{pr}_{1}\left(\widehat{f}^{n}(x)-x\right): x \in \mathbf{R}^{2}, n>0\right\}>\operatorname{diam}_{1}(c)+2,
$$

the set $\operatorname{sing}(\mathcal{F})$ is inessential.

Proof. Let $U$ be the connected component of $\mathbf{T}^{2} \backslash \operatorname{sing}(\mathcal{F})$ that contains $c$. Consider a connected component $\widetilde{U} \subset \mathbf{R}^{2}$ of the preimage of $U$ by the projection map $\pi: \mathbf{R}^{2} \rightarrow \mathbf{T}^{2}$. The $\operatorname{set} \operatorname{sing}(\mathcal{F})$ is inessential if $U$ is fully essential, and to show that $U$ is fully essential, it suffices to see that $\widetilde{U}$ intersects $T_{1}(\widetilde{U})$.

Consider a lift $\tilde{c}$ of $c$ contained in $\tilde{U}$. Suppose there is $x \in \mathbf{R}^{2}$ and $n_{0}>0$ such that $\operatorname{pr}_{1}\left(\widehat{f}^{n_{0}}(x)-x\right)>\operatorname{diam}_{1}(c)+2$. Consider an integer translate of $x$ contained in $\left(T_{1}^{-1}(\tilde{c}), \tilde{c}\right)$, and denote it also by $x$. We then have that $\widehat{f}^{n_{0}}(x) \in R\left(T_{1}(\tilde{c})\right)$. As $\left.T_{1}(\tilde{c})\right) \subset T_{1}(\widetilde{U})$, we then have that the isotopy path $\left\{\widehat{f}_{t}(x)\right\}_{t \in\left[0, n_{0}\right]}$ is contained in $\widetilde{U} \cap T_{1}(\widetilde{U})$, and then $\widetilde{U} \cap T_{1}(\widetilde{U}) \neq \emptyset$, as desired.

The following proposition is a consequence of an improved version of a theorem of Moore (Dav86, Theorems 13.4 and 25.1). For a proof, see [KT] (Proposition 1.6).

Proposition 4.3. Let $K \subset \mathbf{T}^{2}$ be a compact inessential filled set such that $f(K)=K$. Then there is a continuous surjection $h: \mathbf{T}^{2} \rightarrow \mathbf{T}^{2}$ and a homeomoprhism $f^{\prime}: \mathbf{T}^{2} \rightarrow \mathbf{T}^{2}$ such that

- $h$ is homotopic to the identity,

- $h f=f^{\prime} h$,

- $K^{\prime}=h(K)$ is totally disconnected, and

- $\left.h\right|_{\mathbf{T}^{2} \backslash K}: \mathbf{T}^{2} \backslash K \rightarrow \mathbf{T}^{2} \backslash K^{\prime}$ is a homeomorphism.

By this proposition we have that if $K=\operatorname{Fill}(\operatorname{sing}(\mathcal{F}))$ is inessential, the homotopy $f_{t}^{\prime}:=h \circ f_{t}$ on $\mathbf{T}^{2}$ from $\left.\operatorname{Id}\right|_{\mathbf{T}^{2}}$ to $f^{\prime}:=f_{1}^{\prime}$ and the foliation $\mathcal{F}^{\prime}=h(\mathcal{F})$ of $\mathbf{T}^{2}$ with singularities are such that $\left(f_{t}^{\prime}\right)_{t}$ is transverse to $\mathcal{F}^{\prime}$, and $\operatorname{sing}\left(\mathcal{F}^{\prime}\right)$ is totally disconnected. As the map $h$ is homotopic to the identity, $\|\widehat{h}-\mathrm{Id}\|<\infty$ for all lift $\widehat{h}: \mathbf{R}^{2} \rightarrow \mathbf{R}^{2}$. By this and as $h f=f^{\prime} h$ it holds the following.

Proposition 4.4. Let $\widehat{f}^{\prime}$ be the canonical lift of $f^{\prime}$ with respect to the isotopy $\left(f_{t}^{\prime}\right)_{t}$. Then:

1. $\rho(x, \widehat{f})=v$ if and only if $\rho\left(h(x), \widehat{f}^{\prime}\right)=v$,

2. Let $x \in \mathbf{R}^{2}$. There is $N>0$ such that $\operatorname{pr}_{1}\left(\widehat{f}^{n N}(x)-x\right)>n$ for all $n>0$ if and only if there is $N^{\prime}>0$ such that $\operatorname{pr}_{1}\left(\widehat{f}^{\prime n N^{\prime}}(x)-x\right)>n$ for all $n>0$.

\subsection{The circloid $\mathcal{C} \subset \mathbf{R} \times \mathbf{T}^{1}$.}

Consider the lift $\widetilde{\mathcal{F}}$ of $\mathcal{F}$ to $\mathbf{R} \times \mathbf{T}^{1}$. We will show that there exists an essential circloid $\mathcal{C} \subset \mathbf{R} \times \mathbf{T}^{1}$ which is the union of leaves and singularities of $\widetilde{\mathcal{F}}$. We begin by showing that the leaves of $\widetilde{\mathcal{F}}$ are uniformly bounded horizontally.

Claim 4.5. There is $m>0$ such that for every leaf $l \in \widehat{\mathcal{F}}$, we have $\operatorname{diam}\left(\operatorname{pr}_{1}(l)\right)<m$.

Proof. By Lemma 4.1, there exist two closed vertcal loops $c_{1}, c_{2} \subset \mathbf{T}^{2}$, such that they are positively transverse to $\mathcal{F}, c_{1}$ is oriented upwards, and $c_{2}$ is oriented downwards. Consider lifts $\widehat{c}_{1}, \widehat{c}_{2} \subset \mathbf{R}^{2}$ of $c_{1}$ and $c_{2}$, respectively, such that $\widehat{c}_{1} \subset L\left(\widehat{c}_{2}\right)$, and such that the open strip 
$\left(\widehat{c}_{1}, \widehat{c}_{2}\right) \subset \mathbf{R}^{2}$ contains a fundamental domain $D$ of the projection $\mathbf{R}^{2} \rightarrow \mathbf{T}^{2}$. Finally, consider lifts $\widehat{c}_{0}$ and $\widehat{c}_{3}$ of $c_{2}$ and $c_{1}$, respectively, such that $\widehat{c}_{0} \subset L\left(\widehat{c}_{1}\right)$ and $\widehat{c}_{2} \subset L\left(\widehat{c}_{3}\right)$.

We will show that every leaf $l \in \widehat{\mathcal{F}}$ that intersects the fundamental domain $D$ is contained in the open strip $\left(\widehat{c}_{0}, \widehat{c}_{3}\right)$. Setting $m=\operatorname{diam}_{1}\left(\left(\widehat{c}_{0}, \widehat{c}_{3}\right)\right)$, this will prove the claim. Suppose then that a leaf $l \in \widehat{\mathcal{F}}$ intersects $D$ in a point $x$. Parametrize $l: \mathbf{R} \rightarrow \mathbf{R}^{2}$ according to its orientation, and let $l_{1}=l_{[0, \infty)}$ and $l_{2}=\left.l\right|_{(-\infty, 0)}$. As $c_{1}$ and $c_{2}$ are positively transverse to $\mathcal{F}$, the leaves $\widehat{c}_{1}$ and $\widehat{c}_{2}$ are positively transverse to $\widehat{\mathcal{F}}$, and also $\hat{c}_{1}$ is oriented upwards and $\hat{c}_{2}$ is oriented downwards. Therefore, the curve $l_{1} \subset l \in \mathcal{F}$ is contained in $\left(\widehat{c}_{1}, \widehat{c}_{2}\right)$. Analogously, as $\hat{c}_{0}$ is oriented downwards and $\hat{c}_{3}$ is oriented upwards, the curve $l_{2}$ is contained in $\left(\widehat{c}_{0}, \widehat{c}_{3}\right)$, and therefore $l=l_{1} \cup l_{2}$ is contained in $\left(\widehat{c}_{0}, \widehat{c}_{3}\right)$. As we mentioned, this proves the claim.

We now state a result from Jäg09b concerning the construction of circloids. We say that a set $U \subset \mathbf{R} \times \mathbf{T}^{1}$ is an upper generating set if $U$ is bounded to the left and $\bar{U}$ is essential. Its associated lower component $\mathcal{L}(U)$ is the connected component of $\mathbf{R} \times \mathbf{T}^{1} \backslash \bar{U}$ that is unbounded to the left. Similarily, we call a set $L \subset \mathbf{R} \times \mathbf{T}^{1}$ a lower generating set if $L$ is bounded to the right and $\bar{L}$ is essential, and its associated upper component $\mathcal{U}(L)$ is the connected component of $\mathbf{R} \times \mathbf{T}^{1} \backslash \mathcal{U}$ that is unbounded to the right. Observe that if $U$ is upper (lower) generating, then $\mathcal{L}(U)$ is lower (upper) generating, and then the expressions $\mathcal{U L}(U), \mathcal{L U}(L), \mathcal{L U} \mathcal{L}(U)$, etc. make sense.

Lemma 4.6 (Lemma 3.2 from Jäg09b). Suppose that $U$ is an upper generating set. Then $\mathcal{C}^{-}(U):=\mathbf{R} \times \mathbf{T}^{1} \backslash(\mathcal{U L}(U) \cup \mathcal{L U} \mathcal{L}(U))$ is a circloid. Similarly, if $L$ is a lower generating set, then $\mathcal{C}^{+}:=\mathbf{R} \times \mathbf{T}^{1} \backslash(\mathcal{L U}(L) \cup \mathcal{U} \mathcal{L U}(L))$ is a circloid.

In the following claim we use this lemma to find a circloid $\mathcal{C} \subset \mathbf{R} \times \mathbf{T}^{1}$ formed by leaves and singularities of $\widetilde{\mathcal{F}}$.

Claim 4.7. There exists an essential circloid $\mathcal{C} \subset \mathbf{R} \times \mathbf{T}^{1}$ which is a union of leaves and singularities of $\widetilde{\mathcal{F}}$.

Proof. Let $C \subset \mathbf{R} \times \mathbf{T}^{1}$ be a vertical straight circle. Consider its saturation by the foliation $\widetilde{\mathcal{F}}$, i.e., the set $S(C) \subset \mathbf{R} \times \mathbf{T}^{1}$ which is the union of all the leaves and singularities of $\widetilde{\mathcal{F}}$ that intersect $C$. The set $\overline{S(C)}$ is then compact, because by Claim 4.5 $S(C)$ is bounded. It is also essential, as it contains $C$, and therefore it is an upper generating set. The foliation $\widetilde{\mathcal{F}}$ may be embedded in a flow $\phi_{t}$ Whi33, Whi41, and the set $S(C)$ is by definition totally invariant by $\phi_{t}$. Then, the sets $\mathcal{L}(S(C)), \mathcal{U L}(S(C))$, etc. and their complements are also totally invariant by $\phi_{t}$.

By Lemma 4.6 the set $\mathcal{C}:=\mathcal{C}^{-}(S(C))$ is a circloid, and by the above it is totally invariant by $\phi_{t}$. In other words, $\mathcal{C}$ is a union of leaves and singularities of $\widetilde{\mathcal{F}}$, as desired.

We say that a leaf $\gamma \subset \mathbf{R} \times \mathbf{T}^{1}$ is bounded if any lift of $\gamma$ to $\mathbf{R}^{2}$ is bounded.

Claim 4.8. If $\mathcal{C}$ contains a singularity of $\widetilde{\mathcal{F}}$, then the leaves of $\widetilde{\mathcal{F}}$ contained in $\mathcal{C}$ are bounded.

Proof. We know by Claim 4.5 that such leaves are bounded horizontally. By contradiction, suppose on the contrary that there exists a leaf $\gamma$ of $\widetilde{\mathcal{F}}$ contained in $\mathcal{C}$ which is unbounded vertically. It is therefore easy to see that the omega-limit $\omega(\gamma) \subset \mathbf{R} \times \mathbf{T}^{1}$ is essential. As $\mathcal{C}$ is compact, $\omega(\gamma)$ is contained in $\mathcal{C}$. As $\mathcal{C}$ contains a singularity of $\widetilde{\mathcal{F}}, \omega(\gamma) \neq \gamma$ and $\omega(\gamma)$ is a proper essential subset of $\mathcal{C}$, which contradicts the fact that $\mathcal{C}$ is a circloid. This proves the claim.

For further reference, we restate Claim 4.8 explicitly in terms of $\widehat{\mathcal{F}}$.

Claim 4.9. If $\mathcal{C}$ contains a singularity of $\widetilde{\mathcal{F}}$, the leaves of $\widehat{\mathcal{F}}$ contained in $\pi^{-1}(\mathcal{C}) \subset \mathbf{R}^{2}$ are bounded (where $\pi: \mathbf{R}^{2} \rightarrow \mathbf{R} \times \mathbf{T}$ denotes the canonical projection). 


\subsection{Definition of the bound $M=M(f)$ from Theorem D.}

Recall that by Lemma 4.2 if $c \subset \mathbf{T}^{2} \backslash \operatorname{sing}(\mathcal{F})$ is any essential vertical loop, and if

$$
\sup \left\{\operatorname{pr}_{1}\left(\widehat{f}^{n}(x)-x\right): x \in \mathbf{R}^{2}, n \geq 0\right\}>\operatorname{diam}_{1}(c)+2,
$$

we have that $\operatorname{sing}(\mathcal{F})$ is inessential. By a vertical Brouwer line we mean a line $\ell \subset \mathbf{R}^{2}$ such that its (canonical) projection to $\mathbf{R} \times \mathbf{T}^{1}$ is an essential simple closed curve.

The definition of $M$ is done separately in two cases.

Case 1: there exists a vertical Brouwer line $\ell$ for $\widehat{f}$. In this case define

$$
M=\operatorname{diam}_{1}(\ell)+3 \text {. }
$$

What will be used of this definition is that it guarantees that, if there are $z$ and $n$ such that $\operatorname{pr}_{1}\left(\widehat{f}^{n}(z)-z\right)>M$, then $\operatorname{sing}(\mathcal{F})$ is inessential, and also

$$
\widehat{f}^{n}(\ell) \cap R\left(T_{1}^{3}(\ell)\right) \neq \emptyset .
$$

Case 2: there is no vertical Brouwer line for $\widehat{f}$. In this case we may assume that if $(0, a),(0, b) \in$ $\rho(\widehat{f})$ are as in Theorem $\mathrm{D}$, then for any rational $r \in(a, b)$, the vector $(0, r) \in \rho(\widehat{f})$ is realized by a periodic orbit. This is because of the following result, which is proved in Dáv13] although it was already known in the folklore and is essentially contained in Cal05.

Proposition 4.10. If there is $r \in(a, b)$ such that $(0, r) \in \rho(\widehat{f})$ is not realized by a periodic orbit, then there is a vertical Brouwer line for $\widehat{f}$.

As we are in the case that there is no vertical Brouwer line for $\widehat{f}$, we may then assume there is $p \in \mathbf{R}^{2}$ be such that $\pi(p) \in \mathbf{T}^{2}$ is periodic for $f$ and such that

$$
\rho(\pi(p), \widehat{f})=(0, r), \quad r<0 .
$$

Now, fix a connected component $\mathcal{C}_{0} \subset \mathbf{R}^{2}$ of $\pi^{-1}(\mathcal{C})$. Consider an integer translate of $p$, denoted also by $p$, such that there is a straight vertical line $l_{1}$ oriented upwards with $\mathcal{C}_{0} \subset L\left(l_{1}\right)$ and $\left\{\widehat{f}_{t}(p): t \in \mathbf{R}\right\} \subset R\left(l_{1}\right)$.

Let $n_{1}>0$ be such that there is a straight vertical line $l_{2}$ oriented upwards with

$$
\left\{\widehat{f}_{t}(p): t \in \mathbf{R}\right\} \subset L\left(l_{2}\right) \text { and } R\left(l_{2}\right) \supset T_{1}^{n_{1}}\left(\mathcal{C}_{0}\right) \text {. }
$$

For $i \in \mathbf{Z}$, denote

$$
\mathcal{C}_{i}=T_{1}^{n_{1} i}\left(\mathcal{C}_{0}\right)
$$

and note that

$$
\left\{\widehat{f}_{t}(p): t \in \mathbf{R}\right\} \subset\left(l_{1}, l_{2}\right) \subset R\left(\mathcal{C}_{0}\right) \cap L\left(\mathcal{C}_{1}\right) .
$$

Fix $c \subset \mathbf{T}^{2} \backslash \operatorname{sing}(\mathcal{F})$ an essential vertical loop (which exists by Lemma 4.1), and define

$$
M=\operatorname{diam}_{1}(\mathcal{C})+\operatorname{diam}_{1}(c)+2 n_{1}
$$

What will be used of this definition is that it guarantees that if there are $z$ and $n$ such that $\operatorname{pr}_{1}\left(\widehat{f}^{n}(z)-z\right)>M$, then $\operatorname{sing}(\mathcal{F})$ is inessential and

$$
\widehat{f}^{n}\left(\mathcal{C}_{i}\right) \cap \mathcal{C}_{i+2} \neq \emptyset
$$

for any $i$. 


\subsection{Collapsing the connected components of $\operatorname{sing}(\widehat{\mathcal{F}})$.}

The main part of the proof of Theorem D is contained in Sections 4.5 and 4.6. To prove the theorem, in those sections it will be assumed that there is $x \in \mathbf{R}^{2}$ and $n>0$ such that $\operatorname{pr}_{1}\left(\widehat{f}^{n}(x)-x\right)>M$. In such case, by the construction of $M$ in last section the $\operatorname{set} \operatorname{sing}(\mathcal{F})$ is inessential. Therefore, by Proposition 4.3 and by the remark following it, we have that there is a surjection $h: \mathbf{R}^{2} \rightarrow \mathbf{R}^{2}$ such that the isotopy $f_{t}^{\prime}=h \circ f_{t}$ is transverse to the foliation $\mathcal{F}^{\prime}=h(\mathcal{F})$, and $\operatorname{sing}\left(\mathcal{F}^{\prime}\right)$ is totally disconnected.

Consider the canonical lift $\widehat{f}^{\prime}$ of $f^{\prime}=f_{1}^{\prime}$ with respect to the isotopy $\left(f_{t}^{\prime}\right)_{t}$. Note that if $l \subset \mathbf{T}^{2}$ is an essential closed curve such that a lift $\ell$ of $l$ to $\mathbf{R}^{2}$ is a vertical Brouwer curve for $\widehat{f}$, then $l \cap \operatorname{Fix}(f)=\emptyset$ (unless $l \subset \operatorname{Fix}(f)$, case in which $\widehat{f}(\ell)$ is a translate of $\ell$ and Theorem D easily follows). Let $\widehat{h}: \mathbf{R}^{2} \rightarrow \mathbf{R}^{2}$ be a lift of $h$. As $\left.h\right|_{\mathbf{T}^{2} \backslash \operatorname{Fix}(f)}$ is a homeomorphism, a curve $\ell$ is a vertical Brouwer curve for $\widehat{f}$ iff $\widehat{h}(\ell)$ is a vertical Brouwer curve for $\widehat{f}^{\prime}$. We conclude that if there are $x, n$ such that $\operatorname{pr}_{1}\left(\widehat{f}^{n}(x)-x\right)>M$, the properties of $M$ that will be used, namely $(5)$ and 10 , are still valid for $\widehat{f}^{\prime}$ :

- if there is a vertical Brouwer curve $\ell$ for $\widehat{f}$, then $\widehat{f}^{\prime n}(\widehat{h}(\ell)) \cap T_{1}^{3}(\widehat{h}(\ell)) \neq \emptyset$,

- if there is no vertical Brouwer curve for $\widehat{f}$, then $\widehat{f}^{\prime n}\left(\widehat{h}\left(\mathcal{C}_{i}\right)\right) \cap \widehat{h}\left(\mathcal{C}_{i+2}\right) \neq \emptyset$.

By this and by Proposition 4.4 from now on we may make the following assumption.

Assumption 4.11. The set $\operatorname{sing}(\mathcal{F})$ is totally disconnected.

\subsection{Case that $\mathcal{C}$ does not contain singularities.}

Observe that in this case, the lifts $\mathcal{C}_{i}$ from (8) are Brouwer curves for $\widehat{f}$. Through this section, we denote

$$
\ell=\mathcal{C}_{0} .
$$

Consider the constant $M>0$ constructed in Section 4.3 . To prove Theorem $\mathrm{D}$ in this case, through all of this section we assume that there is $x \in \mathbf{R}^{2}$ and $N_{1}>0$ such that $\operatorname{pr}_{1}\left(\widehat{f}^{N_{1}}(x)-x\right)>$ $M$ and we will show that there is $z \in \mathbf{R}^{2}$ and $N>0$ such that $\operatorname{pr}_{1}\left(\widehat{f}^{n N}(z)-z\right)>n$ for all $n \in \mathbf{N}$.

We begin by giving an idea of the proof.

\subsubsection{Idea of the proof.}

Recall that, by hypothesis, $\rho(\widehat{f})$ contains two points $(0, a)$ and $(0, b)$ such that $a<0<b$. We will work under the following assumption, which will be justified in Section 4.5.2.

Assumption 4.12. Every rational point contained in the segment $\{0\} \times(a, b)$ is realized by a periodic orbit.

Under this assumption, we will construct a set $\mathcal{V} \subset R(\ell)$ such that it separates $R(\ell)$ and an iterate of $\mathcal{V}$ is contained in $R\left(T_{1}(\ell)\right)$. Denote by $\mathcal{V}_{i, j}$ its integer translates $\mathcal{V}_{i, j}=T_{1}^{i} T_{2}^{j}(\mathcal{V})$, $i, j \in \mathbf{Z}$. We will define the property of a curve $\gamma$ having good intersection with one of the sets $\mathcal{V}_{i, j}$. By now, we can think of that property as meaning that the $\operatorname{arc} \gamma$ 'traverses' $\mathcal{V}_{i, j}$ (cf. Fig. 5).

We will prove that there is $N_{2}>0$ such that, if a curve $\gamma$ has good intersection with $\mathcal{V}_{i, j}$ for some $i, j$, then $\widehat{f}^{N_{2}}(\gamma)$ has good intersection with $\mathcal{V}_{i+1, j^{\prime}}$, for some $j^{\prime} \in \mathbf{Z}$ (cf. Fig. 6). We 
will verify also that $\ell$ has good intersection with $\mathcal{V}$. An easy induction then will give us that, for any $n>0$ there is $j_{n} \in \mathbf{Z}$ such that $\widehat{f}^{n N_{2}}(\ell)$ has good intersection with $\mathcal{V}_{n, j_{n}}$. In particular,

$$
\widehat{f}^{n N_{2}}(\ell) \cap R\left(T_{1}^{n}(\ell)\right) \supset \widehat{f}^{n\left(N_{2}\right)}(\ell) \cap V_{n, j_{n}} \neq \emptyset \quad \forall n \in \mathbf{N} .
$$

This will easily imply the desired conclusion, namely, there are $z \in \mathbf{R}^{2}, N>0$ such that $\operatorname{pr}_{1}\left(\widehat{f}^{n N}(z)-z\right)>n$ for all $n>0$.

\subsubsection{Assumption 4.12,}

In this section we show how Assumption 4.12 is justified by the results of [Dáv13]. We will see that if there is a rational point in the segment $\{0\} \times[a, b] \subset \rho(\widehat{f})$ which is not realized by a periodic orbit, then the conclusions of Theorem D hold.

Suppose there is $p / q \in \mathbf{Q} \cap[a, b]$, with $p$ and $q$ coprime integers, and such that $(0, p / q) \in \rho(\widehat{f})$ is not realized by a periodic orbit. We now show that we may assume that $(0, p / q)=(0,0)$. Let $\widehat{g}=T_{2}^{-p} \widehat{f}^{q}$, and note that $(0,0) \in \rho(\widehat{g})$ is not realized by a periodic orbit of $f$ (see Section 2.2 for the basic properties of the rotation set). Note also the following:

- if there are $x \in \mathbf{R}^{2}, n \in \mathbf{N}$ s.t. $\operatorname{pr}_{1}\left(\widehat{g}^{n}(x)-x\right)>M$, then $\operatorname{pr}_{1}\left(\widehat{f}^{n^{\prime}}(x)-x\right)>M$ with $n^{\prime}=n q$

- if there are $x \in \mathbf{R}^{2}, N \in \mathbf{N}$ s.t. $\operatorname{pr}_{1}\left(\widehat{g}^{n N}(x)-x\right)>n$ for all $n \in \mathbf{N}$, then $\operatorname{pr}_{1}\left(\widehat{f}^{n N^{\prime}}(x)-x\right)>$ $n$ for all $n \in \mathbf{N}$, with $N^{\prime}=q N$.

Therefore, without loss of generality we may assume that the rational point in $\rho(\widehat{f})$ which is not realized by a periodic orbit is $(0,0)$.

In Proposition 5.2 of [Dáv13] it is shown ${ }^{2}$ that the fact that $\rho(\widehat{f})$ contains $(0, a),(0, b)$, $a<0<b$, implies that there exists a compact vertical leave $l \subset \mathbf{T}^{2}$ of the foliation $\mathcal{F}$ (this was already known in the folklore).

For $i \in \mathbf{N}$, fix a lift $\hat{l}_{0} \subset \mathbf{R}^{2}$ of the curve $l$ and let $\hat{l}_{1}=T_{1}\left(\hat{l}_{0}\right)$. Note that $\hat{l}_{0}$ and $\hat{l}_{1}$ are Brouwer curves for $\widehat{f}$.

In Dáv13] it is shown that, if there is $n>0$ such that $\widehat{f}^{n}\left(\hat{l}_{0}\right) \cap R\left(\hat{l}_{1}\right) \neq \emptyset$, then $\max \left(\operatorname{pr}_{1}(\rho(\widehat{f}))\right)>$ 0 (cf. Main Lemma 6.14 and Claim 6.15 in that article, where it is proved a stronger result). In this case, Frank's Theorem 2.3 implies that there is a periodic point for $f$ which rotates rightwards, that is, there are $z \in \mathbf{R}^{2}$ and $N>0$ such that $\operatorname{pr}_{1}\left(\widehat{f}^{n N}(z)-z\right)>n$ for all $n \in \mathbf{N}$.

On the other hand, by definition of $M=M(f)>0$ we have that if there are $x \in \mathbf{R}^{2}$ and $n>0$ such that $\operatorname{pr}_{1}\left(\widehat{f}^{n}(x)-x\right)>M$, then $\widehat{f}^{n}\left(\mathcal{C}_{i}\right) \cap T_{1}\left(\mathcal{C}_{i}\right) \neq \emptyset$ for all $i$ (cf. Section 4.3), which in particular implies that $\widehat{f}^{n}\left(\hat{l}_{0}\right) \cap \hat{l}_{1} \neq \emptyset$ (recall that $\mathcal{C}$ is also a compact vertical leaf of $\mathcal{F}$ ).

We conclude that if there are $x \in \mathbf{R}^{2}, n>0$ such that $\operatorname{pr}_{1}\left(\widehat{f}^{n}(x)-x\right)>M$, then $\widehat{f}^{n}\left(\hat{l}_{0}\right) \cap$ $R\left(\hat{l}_{1}\right) \neq \emptyset$ and then there are $z \in \mathbf{R}^{2}$ and $N>0$ such that $\operatorname{pr}_{1}\left(\hat{f}^{n N}(z)-z\right)>n$ for all $n \in \mathbf{N}$, in the case that $(0,0) \in \rho(\widehat{f})$ is not realized by a periodic orbit. That is, Theorem $\mathrm{D}$ holds in the case that $\mathcal{C}$ does not contain singularities and $(0,0) \in \rho(\widehat{f})$ is not realized by a periodic orbit, as we wanted.

\subsubsection{Statement of the main lemma and proof of Theorem $\mathrm{D}$ when $\mathcal{C}$ contains no singularities.}

The following main and technical lemma gives us the precise properties of the set $\mathcal{V}$ mentioned in 4.5 .1

\footnotetext{
${ }^{2}$ That proposition is stated under the hypothesis that $\rho(\widehat{f})$ is a segment. However, that is used only guarantee the existence of $x, y \in \mathbf{T}^{2}$ with $\rho(x, \widehat{f})=(0, a), \rho(y, \widehat{f})=(0, b)$ and $a<0<b$, which is within our hypotheses.
} 
Recall that $\ell=\mathcal{C}_{0}$ is a Brouwer curve for $\widehat{f}$ and that $L_{\infty}^{i}, R_{\infty}^{i}$ denote the stable and unstable sets, respectively, of the maximal invariant set in each strip $\left(\ell_{i}, \ell_{i+1}\right)=\left(T_{1}^{i}(\ell), T_{1}^{i+1}(\ell)\right)(\mathrm{cf}$. Section 2.5.

Lemma 4.13. The following hold (cf. Fig. 5):

1. There exist sets $L_{1}, L_{2}, R_{1}, R_{2} \subset R(\ell)$ such that:

(a) for $i=1,2, L_{i} \subset L_{\infty}^{1} \backslash\left(L_{\infty}^{0} \cup R_{\infty}^{1}\right)$, and $\widehat{f}^{n}\left(L_{i}\right) \subset\left(\ell_{1}, \ell_{2}\right)$ for all $n>0$ sufficiently large,

(b) $R_{i} \subset R_{\infty}^{1}$, and then $\widehat{f}^{-n}\left(R_{i}\right) \subset R\left(\ell_{1}\right)$ for all $n \geq 0$,

(c) $\rho\left(\bar{L}_{i}, \widehat{f}\right)=\left\{r_{i}\right\}$ for some $r_{1} \neq r_{2}$, and in particular $\bar{L}_{1} \cap \bar{L}_{2}=\emptyset$,

(d) the sets $L_{i}$ and $R_{i}$ are connected, the $\bar{L}_{i}$ are compact, the $R_{i}$ are unbounded to the right, and the sets $F_{i}=L_{i} \cup R_{i}$ separate $R(\ell)$,

2. There is a set $\mathcal{V} \subset R(\ell)$ such that:

(a) $\mathcal{V}$ is of the form $\mathcal{V}=\left(V_{1} \cap V_{2}\right) \cup R_{1}$, for some open sets $V_{1}, V_{2} \subset R(\ell)$ such that $L_{1} \subset V_{2}, L_{2} \subset V_{1}$, and $\partial V_{i} \subset \ell \cup F_{i}$,

(b) $\partial \mathcal{V} \subset F_{1} \cup F_{2} \cup \ell, \emptyset \neq V_{1} \cup V_{2} \subset \operatorname{int}(\mathcal{V})$, and $\mathcal{V}$ separates $R(\ell)$,

(c) there is $n_{1}>0$ such that $\widehat{f}^{n_{1}}(\overline{\mathcal{V}}) \subset R\left(\ell_{1}\right)$,

(d) $\overline{\mathcal{V}} \cap\left[\ell, \ell_{1}\right]$ is compact,

(e) the set $J:=\partial \mathcal{V} \cap \ell$ is a non-degenerate arc such that $J \cap L_{\infty}^{0}=\emptyset, J(0) \in \bar{L}_{2}$, and $J(1) \in \bar{L}_{1}$.

The proof of this lemma will be given in Section 4.5.4

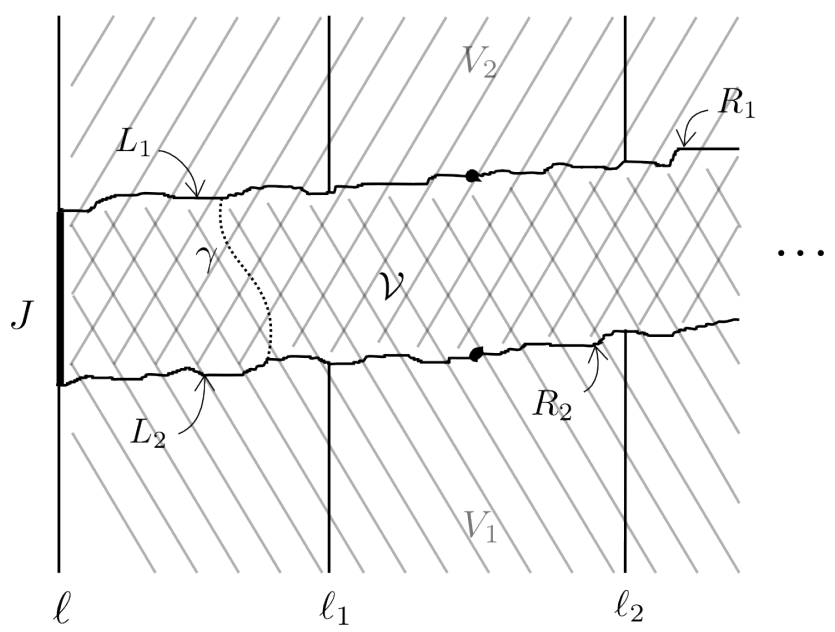

Figure 5: The sets $L_{i}, R_{i}, \mathcal{V}, J$ from Lemma 4.13, and an illustration of an $\operatorname{arc} \gamma$ which has good intersection with $\mathcal{V}$.

Notation 4.14. For $i \in \mathbf{N}_{0}$ and $j \in \mathbf{Z}$ we denote $\ell_{i}=T_{1}^{i}(\ell)$, and $\mathcal{V}_{i, j}=T_{1}^{i} T_{2}^{j}(\mathcal{V})$. Similarily, for $k=1,2$, we set $L_{k}^{i, j}=T_{1}^{i} T_{2}^{j}\left(L_{k}\right), R_{k}^{i, j}=T_{1}^{i} T_{2}^{j}\left(R_{k}\right)$ and $F_{k}^{i, j}=T_{1}^{i} T_{2}^{j}\left(F_{k}\right)$. 
The main induction step in this section is given by Proposition 4.16 below. In order to state it we need the following definition.

Definition 4.15. Let $i \in \mathbf{N}_{0}, j \in \mathbf{Z}$. We say that a curve $\gamma$ has good intersection with $\mathcal{V}_{i, j}$ if the following hold (see Fig. 5):

- $\gamma \cap R_{\infty}^{i+1}=\emptyset$,

- one endpoint of $\gamma$ lies in $\bar{L}_{1}^{i, j}$ and the other in $\bar{L}_{2}^{i, j}$,

- $\operatorname{int}(\gamma) \subset \operatorname{int}\left(\mathcal{V}_{i, j}\right) \cup J$, where $J \subset \ell$ is the arc from Lemma 4.13 2e

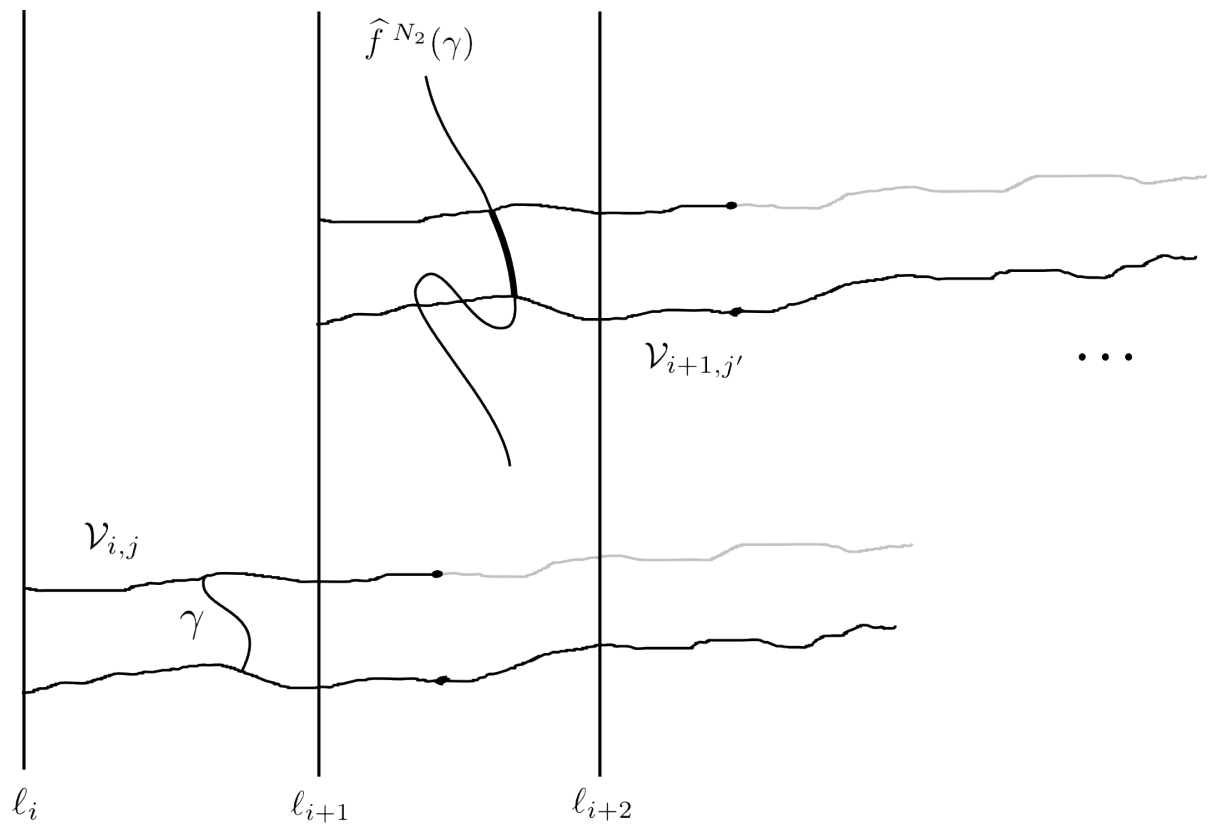

Figure 6: Illustration of Proposition 4.16.

Note that, in particular, if an arc $\gamma$ has good intersection with $\mathcal{V}_{i, j}$ for some $i, j$, then $\gamma \subset \bar{R}\left(\ell_{i}\right)$

Proposition 4.16. There is $N_{2}>0$ such that:

1. if an arc $\gamma$ has good intersection with $\mathcal{V}_{i, j}$ for some $i, j \in \mathbf{Z}$, then $\widehat{f}^{N_{2}}(\gamma)$ contains an arc which has good intersection with $\mathcal{V}_{i+1, j^{\prime}}$, for some $j^{\prime} \in \mathbf{Z}$ (see Fig. 6),

2. the arc $J \subset \ell$ from Lemma 4.13 2e is such that $\widehat{f}^{N_{2}}(J)$ has good intersection with $\mathcal{V}_{1, j}$, for some $j \in \mathbf{Z}$.

Before proving this proposition, we use it to prove Theorem D.

Proof of Theorem $D$ when $\mathcal{C}$ contains no singularities. Let $n_{0}>0$ be such that $\left.\min \left\{\operatorname{pr}_{1}\left(\ell_{n_{0}}\right)\right)\right\}>$ $\max \left\{\operatorname{pr}_{1}(\ell)\right\}+1$. By proposition 4.16 we easily get by induction that $\widehat{f}^{n_{0}} N_{2}(J)$ contains an arc $\beta_{1}$ which has good intersection with $\mathcal{V}_{n_{0}, j_{1}}$, for some $j_{1} \in \mathbf{Z}$. In particular, $\beta_{1} \subset \bar{R}\left(\ell_{n_{0}}\right)$. 
Let

$$
\bar{\beta}_{1}=\widehat{f}^{-n_{0} N_{2}}\left(\beta_{1}\right) \subset J .
$$

Analogously, by induction using item 1 of Proposition 4.16 it easily follows that there is a sequence of $\operatorname{arcs} \bar{\beta}_{n} \subset \bar{\beta}_{1}$ such that $\bar{\beta}_{k} \subset \bar{\beta}_{l}$ if $k>l$, and such that for all $n \geq 1$, the $\operatorname{arc} \hat{f}^{n \cdot n_{0} N_{2}}\left(\bar{\beta}_{n}\right)$ has good intersection with $\mathcal{V}_{n \cdot n_{0}, j_{n}}$ for some $j_{n} \in \mathbf{Z}$, and in particular, $\widehat{f}^{n \cdot n_{0} N_{2}}\left(\bar{\beta}_{n}\right) \subset \bar{R}\left(\ell_{n \cdot n_{0}}\right)$.

If $z \in \cap_{n \geq 0} \bar{\beta}_{n}$, then for every $n \geq 1, \widehat{f}^{n \cdot n_{0} N_{2}}(z) \in \bar{R}\left(\ell_{n \cdot n_{0}}\right)$, and in particular, by the choice of $n_{0}$,

$$
\operatorname{pr}_{1}\left(\widehat{f}^{n \cdot n_{0} N_{2}}(z)-z\right)>n \quad \text { for all } n>0 .
$$

Setting $N=n_{0} N_{2}$, this finishes the proof.

We now prove Proposition 4.16 .

Proof of Proposition 4.16. Without loss of generality, assume that $i=j=0$. Let $K=$ $\operatorname{diam}_{2}\left(\mathcal{V} \cap\left(\ell, \ell_{1}\right)\right)+1$, which by item $2 \mathrm{~d}$ from Lemma 4.13 is finite, and note that, by the periodicity of $\widehat{f}$,

$$
K=\operatorname{diam}_{2}\left(\mathcal{V}_{i, j} \cap\left(\ell_{i}, \ell_{i+1}\right)\right)+1 \quad \forall i, j .
$$

Consider the sets $L_{1}, L_{2}$ from Lemma 4.13 and let $n_{1}$ be as in item $2 \mathrm{c}$ of such lemma. By item 1 a we have that there is $n_{2}>n_{1}$ such that

$$
\widehat{f}^{n}\left(L_{1} \cup L_{2}\right) \subset\left(\ell_{1}, \ell_{2}\right) \quad \forall n \geq n_{2} .
$$

By item $1 \mathrm{c}$ we have that $\rho\left(\bar{L}_{i}, \widehat{f}\right)=r_{i}$ for some $r_{1} \neq r_{2}$, and therefore there is $N_{2}>n_{2}$ such that

$$
d\left(\operatorname{pr}_{2}\left(\widehat{f}^{N_{2}}\left(\bar{L}_{1}\right)\right), \operatorname{pr}_{2}\left(\widehat{f}^{N_{2}}\left(\bar{L}_{2}\right)\right)\right)>K \text {. }
$$

As $\gamma$ has good intersection with $\mathcal{V}$, we have that an endpoint of $\widehat{f}^{N_{2}} \gamma$, say $\widehat{f}^{N_{2}} \gamma(0)$, belongs to $\widehat{f}^{N_{2}}\left(L_{1}\right)$ and $\widehat{f}^{N_{2}} \gamma(1) \in \widehat{f}^{N_{2}}\left(L_{2}\right)$, and by definition of $K$ we have then that, for some $j_{1} \in \mathbf{Z}$, the set $\operatorname{pr}_{2}\left(\mathcal{V}_{1, j_{1}}\right)$ is contained in the interval between $\operatorname{pr}_{2}\left(\widehat{f}^{N_{2}} \gamma(0)\right)$ and $\operatorname{pr}_{2}\left(\widehat{f}^{N_{2}} \gamma(1)\right)$. As the sets $R_{i}$ are contained in $R_{\infty}^{1} \subset R\left(\ell_{2}\right)$ (item $1 \mathrm{~b}$ ) and as the sets $F_{i}^{1, j_{1}}=L_{i}^{1, j_{1}} \cup R_{i}^{1, j_{1}}$ separate $R\left(\ell_{1}\right)$ (by item $2 \mathrm{~b}$ and by the periodicity of $\hat{f}$ ), we obtain that, for $i=1,2$, the points $\widehat{f}^{N_{2}} \gamma(0)$ and $\widehat{f}^{N_{2}} \gamma(1)$ belong to different connected components of $R\left(\ell_{1}\right) \backslash F_{i}^{1, j_{1}}$.

Also, as $\gamma$ has good intersection with $\mathcal{V}$, we have $\widehat{f}^{N_{2}} \gamma \subset \widehat{f}^{N_{2}}(\overline{\mathcal{V}})$, and as $N_{2} \geq n_{2} \geq n_{1}$, by item 2c we obtain

$$
\widehat{f}^{N_{2}} \gamma \subset R\left(\ell_{1}\right) .
$$

Also by the fact that $\gamma$ has good intersection with $\mathcal{V}, \gamma \cap R_{\infty}^{1}=\emptyset$, and then $\widehat{f}^{N_{2}} \gamma \cap R_{\infty}^{1}=\emptyset$ by the invariance of $R_{\infty}^{1}$. As $R_{\infty}^{2} \subset R_{\infty}^{1}$,

$$
\widehat{f}^{N_{2}} \gamma \cap R_{\infty}^{2}=\emptyset
$$

By this, by 111, and as the points $\widehat{f}^{N_{2}} \gamma(0), \widehat{f}^{N_{2}} \gamma(1)$ belong to different connected components of $R\left(\ell_{1}\right) \backslash F_{i}^{1, j_{1}}, i=1,2$, we conclude that the arc $\widehat{f}^{N_{2}} \gamma$ must intersect both sets $L_{1}^{1, j_{1}} \backslash R_{\infty}^{2}$ and $L_{2}^{1, j_{1}} \backslash R_{\infty}^{2}$.

Consider a subarc $\bar{\gamma} \subset \widehat{f}^{N_{2}} \gamma$ with one endpoint in $L_{1}^{1, j_{1}} \backslash R_{\infty}^{2}$, the other endpoint in $L_{2}^{1, j_{1}} \backslash R_{\infty}^{2}$, and minimal with respect to this property. We claim that $\operatorname{int}(\bar{\gamma}) \subset \operatorname{int}\left(\mathcal{V}_{1, j_{1}}\right)$. To see this, note that by item $2 \mathrm{a}$ the set $\mathcal{V}_{1, j_{1}}$ is of the form $\mathcal{V}_{1, j_{1}}=\left(V_{1} \cap V_{2}\right) \cup R_{1}^{1, j_{1}}$ for some open sets $V_{1}, V_{2}$ such that $L_{1}^{1, J_{1}} \subset V_{2} \cup R_{2}, L_{2}^{1, j_{1}} \subset V_{1} \cup R_{1}$ and $\partial V_{i} \subset \ell_{1} \cup F_{i}^{1, j_{1}}$. By the minimality of $\bar{\gamma}$ and by (11), 12) we have

$$
\operatorname{int}(\bar{\gamma}) \cap \partial V_{i} \subset \operatorname{int}(\bar{\gamma}) \cap L_{i}^{1, j_{1}}=\emptyset \quad \text { for } i=1,2 .
$$


Also, by definition $\bar{\gamma}(0) \in L_{1}^{1, j_{1}} \subset V_{2}, \bar{\gamma}(1) \in L_{2}^{1, j_{1}} \subset V_{1}$ and therefore

$$
\operatorname{int}(\bar{\gamma}) \subset V_{1} \cap V_{2} \subset \operatorname{int}\left(\mathcal{V}_{1, j_{1}}\right),
$$

as claimed. By (??) we actually have $\operatorname{int}(\bar{\gamma}) \subset \operatorname{int}\left(\mathcal{V}_{1, j_{1}}\right) \backslash R_{\infty}^{2}$, and then $\bar{\gamma}$ has good intersection with $\mathcal{V}_{1, j_{1}}$ which proves item 1 of the proposition.

We now prove item 2. As the arc $J$ is such that $J \subset \ell, J(0) \in \bar{L}_{2}$ and $J(1) \in \bar{L}_{1}$ then $J$ has good intersection with $\mathcal{V}$. By the previous item, $\widehat{f}^{N_{2}} J$ contains an arc which has good intersection with $\mathcal{V}_{1, j_{1}}$, for some $j_{1} \in \mathbf{Z}$.

\subsubsection{Proof of Lemma 4.13 construction of the sets $L_{i}, R_{i}$ and $\mathcal{V}$.}

4.5.4.1 Some preliminary results. In this section we use Handel's theorem 2.8 to construct the sets $L_{1}, L_{2}$. Recall that by hypothesis on Theorem $\mathrm{D}, \rho(\widehat{f})$ contains two vectors $(0, a)$, $(0, b)$, with $a<0<b$.

Proposition 4.17. For all $r \in[a, b]$ there exists a closed $\widehat{f}$-invariant set $K_{r} \subset\left(\ell, \ell_{1}\right)$ such that $\rho\left(K_{r}, \widehat{f}\right)=\{(0, r)\}$.

Handel's theorem 2.8 is a theorem for annulus maps. Lemma 4.18 below will give us that we may assume we are dealing with an annulus map, to the one we will be able to apply Handel's theorem.

Let $\tilde{f}: \mathbf{R} \times \mathbf{T}^{1} \rightarrow \mathbf{R} \times \mathbf{T}^{1}$ be the lift of $f$ to $\mathbf{R} \times \mathbf{T}^{1}$ such that $\pi \circ \widehat{f}=\tilde{f} \circ \pi$, where $\pi: \mathbf{R}^{2} \rightarrow \mathbf{R} \times \mathbf{T}^{1}$ denotes the canonical projection. Let $\bar{\ell}=\pi(\ell)$ and $\bar{\ell}_{1}=\pi\left(\ell_{1}\right)$. Let $m>0$ be such that $\operatorname{pr}_{1}\left(\left(\widehat{f}^{-1}(\ell), \widehat{f}\left(\ell_{1}\right)\right)\right) \subset[-m, m] \subset \mathbf{R}$, and denote by $A_{m}$ the compact annulus given by $A_{m}=[-m, m] \times \mathbf{T}^{1} \subset \mathbf{R} \times \mathbf{T}^{1}$.

Lemma 4.18. There exists a homeomorphism homotopic to the identity $g: A_{m} \rightarrow A_{m}$ such that:

1. $\left.g\right|_{\left(\bar{\ell}, \bar{\ell}_{1}\right)}=\left.\tilde{f}\right|_{\left(\bar{\ell}, \bar{\ell}_{1}\right)}$.

2. For all $x$ in $R\left(\bar{\ell}_{1}\right)$, the $\omega$-limit $\omega(x, g)$ is contained in $\{m\} \times \mathbf{T}^{1}$, and for all $x$ in $L(\bar{\ell})$, the $\alpha$-limit $\alpha(x, g)$ is contained in $\{-m\} \times \mathbf{T}^{1}$.

3. If $\widehat{g}:[-m, m] \times \mathbf{R} \rightarrow[-m, m] \times \mathbf{R}$ is the lift of $g$ such that $\left.\widehat{g}\right|_{\left(\ell, \ell_{1}\right)}=\left.\widehat{f}\right|_{\left(\ell, \ell_{1}\right)}$, then $\rho\left(\{-m\} \times \mathbf{T}^{1}, \widehat{g}\right)=\left\{\left(0, s_{1}\right)\right\}$ and $\rho\left(\{m\} \times \mathbf{T}^{1}, \widehat{g}\right)=\left\{\left(0, s_{2}\right)\right\}$, for some $s_{1}, s_{2} \in \mathbf{Z}$ with $s_{1}<\min \operatorname{pr}_{2}(\rho(\widehat{f})) \leq a<b \leq \max _{2} \operatorname{pr}_{2}(\rho(\widehat{f}))<s_{2}$.

Proof. For $0<r_{1}<r_{2}$, denote the complex annulus $\mathbf{A}_{r_{1}, r_{2}}=\left\{r e^{i \theta} \in \mathbf{C}: r_{1} \leq r \leq r_{2}\right\}$. Consider the homeomorphism $h: A_{m} \rightarrow \mathbf{A}_{e^{-m}, e^{m}}$ given by $h(x, y)=e^{-x} e^{i y}$, which sends horizontal segments $\left\{y=y_{0}\right\}$ in $A_{m}$ to radial segments $\left\{\theta=e^{y_{0}}\right\}$ in $\mathbf{A}_{e^{-m}}, e^{m}$, and sends the border components $\{-m\} \times \mathbf{T}^{1}$ and $\{m\} \times \mathbf{T}^{1}$ to $\left\{r=e^{m}\right\}$ and $\left\{r=e^{-m}\right\}$, respectively. Up to a change of coordinates (isotopic to the identity), we may assume that the circle $\tilde{f}\left(\bar{\ell}_{1}\right)$ is a straight vertical circle, which corresponds by $h$ to a circle $c_{s}=\{r=s\} \subset \mathbf{A}_{e^{-m}, e^{m}}$.

For $r>0$, denote $\mathbf{D}_{r}=\{z \in \mathbf{C}:|z|<r\}$. Let $F=h \tilde{f} h^{-1}: \mathbf{A}_{e^{-m}, e^{m}} \rightarrow \mathbf{A}_{e^{-m}, e^{m}}$ and note that, as $\tilde{f}^{2}\left(\bar{\ell}_{1}\right) \subset R\left(\tilde{f}\left(\bar{\ell}_{1}\right)\right)$, it holds $F\left(c_{s}\right)=F\left(\partial \mathbf{D}_{s}\right) \subset \mathbf{D}_{s}$. By a Theorem of Moser (Mos65), if $t \in\left(r_{1}, s\right)$ there exists a homeomorphism $\phi: \mathbf{A}_{e^{-m}, s} \rightarrow \mathbf{A}_{e^{-m}, s}$ such that $\left.\phi\right|_{\partial \mathbf{A}_{e^{-m}, s}}=\mathrm{Id}$, and such that $\phi\left(F\left(c_{s}\right)\right)=\partial \mathbf{D}_{t}$. Composing with a twist map with support on an annulus $U \subset \mathbf{A}_{e^{-m}, s}$ which contains $\partial \mathbf{D}_{t}$ we may also suppose that $\left.\phi F\right|_{c_{s}}$ fixes angles, that is, we may 
suppose that $\phi$ is such that for any $z \in c_{s}, \arg (\phi F(z))=\arg (z)$, where $\arg (z)$ denotes the argument of $z$. Let $G_{1}=\phi F$ and note that, as $\phi$ has support on $\mathbf{A}_{e^{-m}, s}$ and as $\tilde{f}\left(\bar{\ell}_{1}\right)=h^{-1}\left(c_{s}\right)$,

$$
\left.G_{1}\right|_{h\left(L\left(\bar{\ell}_{1}\right)\right)}=\left.F\right|_{h\left(L\left(\bar{\ell}_{1}\right)\right)},
$$

and then the map $g_{1}:=h^{-1} G_{1} h: A_{m} \rightarrow A_{m}$ is such that

$$
\left.\left.g_{1}\right|_{L\left(\bar{\ell}_{1}\right)} \equiv \tilde{f}\right|_{L\left(\bar{\ell}_{1}\right)} .
$$

Note that, as $\left.G_{1}\right|_{c_{s}}$ fixes angles, $\left.g_{1}\right|_{\tilde{f}\left(\bar{\ell}_{1}\right)}$ fixes the second coordinate. By an adequate choice of the mentioned twist map, we may assume that, not only $\left.g_{1}\right|_{\tilde{f}\left(\bar{\ell}_{1}\right)}$ fixes the second coordinate, but also, if $\widehat{g}_{1}:[-m, m] \times \mathbf{R} \rightarrow[-m, m] \times \mathbf{R}$ is the lift of $g_{1}$ such that $\left.\left.\widehat{g}_{1}\right|_{L\left(\ell_{1}\right)} \equiv \widehat{f}\right|_{L\left(\ell_{1}\right)}$, we have

$$
\operatorname{pr}_{2}\left(\widehat{g}_{1}(\widehat{z})\right)=\operatorname{pr}_{2}(\widehat{z})+s_{2}
$$

for all $\widehat{z} \in \widehat{f}\left(\ell_{1}\right)$ and where $s_{2} \in \mathbf{Z}, s_{2}>\max _{2}(\rho(\widehat{f})) \geq b>0$.

Consider the restriction $\left.G_{1}\right|_{c_{r_{3}}}: c_{r_{3}} \rightarrow \mathbf{A}_{e^{-m}, e^{m}}$, and radially extend it to an injective map $G_{2}: \mathbf{A}_{e^{-m}, s} \rightarrow \mathbf{A}_{e^{-m}, s}$, so that for every $r e^{i \theta} \in \mathbf{A}_{e^{-m}, s}, G_{2}\left(r e^{i \theta}\right)=\tilde{r} e^{i \theta}$, with $\tilde{r} \leq r$, and with equality iff $r=e^{-m}$. Then, extend $G_{2}$ continuously to $\mathbf{A}_{e^{-m}, e^{m}}$ as $G_{2}(x)=G_{1}(x)$ for $x \in \mathbf{A}_{s, e^{m}}$.

Observe that as $\left.G_{2}\right|_{\mathbf{A}_{s, e} m} \equiv G_{1}$, by $(13)$ we have $\left.\left.G_{2}\right|_{h\left(L\left(\bar{\ell}_{1}\right)\right)} \equiv F\right|_{h\left(L\left(\bar{\ell}_{1}\right)\right)}$, and then the map $g_{2}:=h^{-1} G_{2} h: A_{m} \rightarrow A_{m}$ is such that

$$
\left.\left.g_{2}\right|_{L\left(\bar{\ell}_{1}\right)} \equiv \tilde{f}\right|_{L\left(\bar{\ell}_{1}\right)} .
$$

Let $\widehat{g}_{2}:[-m, m] \times \mathbf{R} \rightarrow[-m, m] \times \mathbf{R}$ be the lift of $g_{2}$ such that $\left.\left.\widehat{g}_{2}\right|_{L\left(\bar{\ell}_{1}\right)} \equiv \widehat{f}\right|_{L\left(\bar{\ell}_{1}\right)}$. As $\left.\left.g_{2}\right|_{R\left(\bar{\ell}_{1}\right)} \equiv g_{1}\right|_{R\left(\bar{\ell}_{1}\right)}$ (by definition) and by $(14)$ we have that $\operatorname{pr}_{2}\left(\widehat{g}_{2}(x)\right)=\operatorname{pr}_{2}(x)$ for $x \in R\left(\ell_{1}\right)$. Also, as $G_{2}\left(\left\{r=e^{-m}\right\}\right)=\left\{r=e^{-m}\right\}$,

$$
\widehat{g}_{2}(x)=x+\left(0, s_{2}\right) \text { for } x \in\{m\} \times \mathbf{R} .
$$

Being the map $G_{2}$ a radial contraction on $\mathbf{A}_{e^{-m}, s}$, we have that for any $z \in \mathbf{A}_{e^{-m}, s}$, $\omega\left(z, G_{2}\right) \subset\left\{r=e^{-m}\right\}$. This implies

$$
\omega\left(x, g_{2}\right) \subset\{m\} \times \mathbf{T}^{1} \quad \text { for any } x \in \bar{R}\left(\bar{\ell}_{1}\right) .
$$

In a symmetric way, we may modify $g_{2}$ to obtain a homeomorphism $g: A_{m} \rightarrow A_{m}$ such that:

- $\left.g\right|_{R(\bar{\ell})}=\left.g_{2}\right|_{R(\bar{\ell})}$,

- $\alpha(x, g) \subset\{-M\} \times \mathbf{T}^{1}$ for any $x \in \bar{L}(\bar{\ell})$,

and such that, if $\widehat{g}: \mathbf{R}^{2} \rightarrow \mathbf{R}^{2}$ is the lift of $g$ such that $\left.\left.\left.\widehat{g}\right|_{\left(\ell, \ell_{1}\right)} \equiv \widehat{g}_{2}\right|_{\left(\ell, \ell_{1}\right)} \equiv \widehat{f}\right|_{\left(\ell, \ell_{1}\right)}$, then

- $\widehat{g}(x)=x+\left(0, s_{1}\right)$

for all $x \in\{-m\} \times \mathbf{R}$ and where $s_{1} \in \mathbf{Z}, s_{1}<\min \operatorname{pr}_{2}(\rho(\widehat{f})) \leq a<0$. By this, and by eqs. 15 , (16), 17) we have that $g$ satisfies items 1 to 3 of the lemma.

We are now ready to prove Proposition 4.17, using Handel's theorem 2.8 
Proof of Proposition 4.17 Let $g: A_{m} \rightarrow A_{m}$ be the annulus homeomorphism given by Lemma 4.18 and let $\widehat{g}:[-m, m] \times \mathbf{R} \rightarrow[-m, m] \times \mathbf{R}$ be the lift of $g$ such that $\left.\left.\widehat{g}\right|_{\left(\ell, \ell_{1}\right)} \equiv \hat{f}\right|_{\left(\ell, \ell_{1}\right)}$. Consider the sets $\rho(\widehat{g})$ and $\rho_{\text {point }}(\widehat{g})$ as subsets of $\{0\} \times \mathbf{R} \subset \mathbf{R}^{2}$. Let $E_{g}$ be the maximal invariant set of $\left(\bar{\ell}, \bar{\ell}_{1}\right)$ for $g$, and let $B=\{-m, m\} \times \mathbf{T}^{1}$. As the curves $\bar{\ell}$ and $\bar{\ell}_{1}$ are free for $g$, items (2) and (3) of Lemma 4.18 imply that

$$
\rho_{\text {point }}(\widehat{g})=\rho_{\text {point }}\left(E_{g} \cup B, \widehat{g}\right)=\rho_{\text {point }}\left(E_{g}, \widehat{g}\right) \cup\left\{\left(0, s_{1}\right),\left(0, s_{2}\right)\right\} .
$$

As $\left.\widehat{g}\right|_{\left(\ell, \ell_{1}\right)}=\left.\widehat{f}\right|_{\left(\ell, \ell_{1}\right)}$,

$$
\rho_{\text {point }}\left(E_{g}, \widehat{g}\right)=\rho_{\text {point }}\left(E_{\tilde{f}}, \widehat{f}\right),
$$

where $E_{\tilde{f}}$ is the maximal invariant set of $\left(\bar{\ell}, \bar{\ell}_{1}\right)$ for $\tilde{f}$. Observe that, by Assumption 4.12 ,

$$
\rho_{\text {point }}\left(E_{\tilde{f}}, \widehat{f}\right) \supset(\{0\} \times[a, b]) \cap \mathbf{Q}^{2},
$$

and then equations 18 and 19 imply

$$
\rho_{\text {point }}(\widehat{g})=\rho_{\text {point }}\left(E_{\tilde{f}}, \widehat{f}\right) \cup\left\{\left(0, s_{1}\right),\left(0, s_{2}\right)\right\} \supset(\{0\} \times[a, b]) \cap \mathbf{Q}^{2} .
$$

By Handel's theorem 2.8, $\rho_{\text {point }}(\widehat{g})$ is closed, and therefore $\rho_{\text {point }}(\widehat{g}) \supset\{0\} \times[a, b]$. Also by that theorem, for all $r \in[a, b]$ there exists a compact set $K_{r} \subset A_{m}$ that is $g$-invariant, and such that $\rho(z, \widehat{g})=r$ for all $z \in K_{r}$.

By item 2 from Lemma 4.18 , the forward $g$-iterates of $\ell_{1}$ accumulate on $\{m\} \times \mathbf{T}^{1}$, the backward $g$-iterates of $\ell$ accumulate on $\{-m\} \times \mathbf{T}^{1}$, and by item 3 of the same lemma, $\rho(x, \widehat{g})=$ $\left\{\left(0, s_{1}\right)\right\}$ for any $x \in\{-m\} \times \mathbf{T}^{1}$ and $\rho(x, \widehat{g})=\left\{\left(0, s_{2}\right)\right\}$ for all $x \in\{m\} \times \mathbf{T}^{1}$, where $s_{1}<$ $a<b<s_{2}$. The fact that $K_{r}$ is $g$-invariant and $r \in[a, b]$ implies then that $K_{r} \subset\left(\bar{\ell}, \bar{\ell}_{1}\right)$. As $\left.g\right|_{\left(\bar{\ell}, \bar{\ell}_{1}\right)}=\left.\widetilde{f}\right|_{\left(\bar{\ell}, \bar{\ell}_{1}\right)}$, we have that $K_{r}$ is $\tilde{f}$-invariant and $\rho\left(K_{r}, \widehat{f}\right)=\rho\left(K_{r}, \widehat{g}\right)=\{(0, r)\}$. This proves the proposition.

We now use Proposition 4.17 to construct the sets $L_{1}, L_{2}$. Such construction will be carried out basically with the use of Corollary 4.20 below.

Lemma 4.19. For all $p \in[a, b]$ there exists $z \in L_{\infty}^{1} \cap R_{\infty}^{1}$ such that, if $C$ is the connected component of $L_{\infty}^{1} \cap R(\ell)$ that contains $z$, then:

1. $\bar{C}$ is compact.

2. $\rho(\bar{C}, \widehat{f})=\{(0, p)\}$.

3. $\bar{C} \cap \ell \neq \emptyset$.

4. $\bar{C} \cap L_{\infty}^{0}=\emptyset$, and therefore there is $n>0$ such that $\widehat{f}^{n}(C) \subset R\left(\ell_{1}\right)$.

Corollary 4.20. There exists an arc $I \subset \ell$, and two points $x, y \in L_{\infty}^{1} \cap R_{\infty}^{1}$ such that, if $L_{x}$ and $L_{y}$ are the connected components of $L_{\infty}^{1} \cap R(\ell)$ that contain $x$ and $y$, respectively, then:

1. $\bar{L}_{x}, \bar{L}_{y}$ are compact.

2. $\operatorname{int}(I) \cap L_{\infty}^{0}=\emptyset$, and the endpoints of I lie in $L_{\infty}^{0}$.

3. $\bar{L}_{i} \cap \operatorname{int}(I) \neq \emptyset$, for $i=x, y$.

4. $\bar{L}_{i} \cap L_{\infty}^{0}=\emptyset$ and there is $n_{1}>0$ such that $\widehat{f}^{n_{1}}\left(\bar{L}_{1} \cup \bar{L}_{2}\right) \subset R\left(\ell_{1}\right)$. 
5. $\rho\left(\bar{L}_{i}, \widehat{f}\right)=\left\{\left(0, r_{i}\right)\right\}$, for $i=x, y$ and some $r_{x} \neq r_{y}$.

Proof. By Lemma 4.19, for any $p \in[a, b]$ there is $z_{p} \in L_{\infty}^{1} \cap R_{\infty}^{1}$ such that, if $C_{z_{p}}$ denotes the connected component of $L_{\infty}^{1} \cap R(\ell)$ that contains $z_{p}$, then

$$
\begin{gathered}
\rho\left(\bar{C}_{z_{p}}, \widehat{f}\right)=\{(0, p)\}, \\
\bar{C}_{z_{p}} \cap L_{\infty}^{0}=\emptyset, \\
\bar{C}_{z_{p}} \cap \ell \neq \emptyset
\end{gathered}
$$

and there is $n_{p}>0$ such that

$$
\widehat{f}^{n_{p}}\left(\bar{C}_{z_{p}}\right) \subset R\left(\ell_{1}\right) .
$$

Let $U=\ell \backslash L_{\infty}^{0}$. As $L_{\infty}^{0}$ is closed, $U$ is a countable union of open arcs. By (23) and as the set $[a, b]$ is uncountable, there must be points $r, s \in[a, b], r \neq s$, points $z_{r}, z_{s} \in L_{\infty}^{1} \cap R_{\infty}^{1}$ and a connected component $U_{0}$ of $U$ such that

$$
\bar{C}_{z_{r}} \cap U_{0} \neq \emptyset \text { and } \bar{C}_{z_{s}} \cap U_{0} \neq \emptyset \text {, }
$$

and, letting $N_{1}=\max \left\{n_{r}, n_{s}\right\}$, by 24 we have

$$
\widehat{f}^{N_{1}}\left(\bar{C}_{z_{r}} \cup \bar{C}_{z_{s}}\right) \subset R\left(\ell_{1}\right) .
$$

Let $I=U_{0}$. By definition, we have that $I$ satisfies item 2 of the corollary. Define $x=z_{r}$ and $y=z_{s}$, so $L_{x}=C_{z_{r}}$ and $L_{y}=C_{z_{s}}$. By item 1 of Lemma 4.19, the sets $\bar{L}_{x}$ and $\bar{L}_{y}$ are compact, and item 1 of the corollary holds. By eqs. (22) and (26), $x$ and $y$ satisfy item (4) of the corollary. By eqs. 25) and 21, items 3 and 5, respectively, hold.

To prove Lemma 4.19 we will need the following.

Lemma 4.21. There does not exist a compact connected set $K$ such that:

1. $K \cap L_{\infty}^{0} \neq \emptyset$, and $K \cap R_{\infty}^{2} \neq \emptyset$,

2. $\rho(K, \widehat{f})$ consists of a point.

Proof. We proceed by contradiction. Suppose that $K$ is a compact connected set such that $K \cap L_{\infty}^{0} \neq \emptyset, K \cap R_{\infty}^{2} \neq \emptyset$, and $\rho(K, \widehat{f})=\left\{\left(0, p_{0}\right)\right\}$, for some $p_{0} \neq 0$ (see Fig. 7). We treat the case $p_{0} \geq 0$, the case $p_{0}<0$ being similar.

By Assumption 4.12 there is a periodic point $y \in \mathbf{T}^{2}$ such that $\rho(y, \widehat{f})=(0, c)$, with $c<0$. We choose a lift $\widehat{y} \in \mathbf{R}^{2}$ of $y$ such that $\widehat{y} \in\left(\ell_{1}, \ell_{2}\right)$, and such that $\widehat{y}$ is above $K$. As $K$ is compact, as $\rho(K, \widehat{f})=\left\{\left(0, p_{0}\right)\right\}$ and as $\rho(y, \widehat{f})=(0, c), c<0$, there is $n_{1}>0$ such that $\widehat{f}^{n}(\widehat{y})$ is below $\widehat{f}^{n}(K)$ for all $n \geq n_{1}$. Observe that the whole orbit of $\widehat{y}$ must be contained in $\left(\ell_{1}, \ell_{2}\right)$, as the curves $\ell_{1}$ and $\ell_{2}$ are Brouwer lines for $\widehat{f}, y$ is periodic, and $\rho(y, \widehat{f})$ is a vertical vector.

Let $z_{1} \in K \cap L_{\infty}^{0}$. Then by definition of $L_{\infty}^{0}, z \in L\left(\widehat{f}^{-n_{1}}\left(\ell_{1}\right)\right)$. Let $\beta_{1}:(-\infty, 0] \rightarrow \mathbf{R}^{2}$ be a proper immersion such that:

- $\beta_{1} \subset L\left(\widehat{f}^{-n_{1}}\left(\ell_{1}\right)\right)$,

- $\beta_{1}(0)=z_{1}$, and

- $-\infty<\inf \operatorname{pr}_{2}\left(\beta_{1}\right)<\sup \operatorname{pr}_{2}\left(\beta_{1}\right)<\infty$.

Now, let $z_{2} \in K \cap R_{\infty}^{2}$, and let $\beta_{2}:[0, \infty) \rightarrow \mathbf{R}^{2}$ be a proper immersion such that: 
- $\beta_{2}(0)=z_{2}$,

- $\beta_{2} \subset R\left(\ell_{2}\right)$, and

- $-\infty<\inf \operatorname{pr}_{2}\left(\beta_{2}\right)<\sup \operatorname{pr}_{2}\left(\beta_{2}\right)<\infty$.

The set $\beta_{1} \cup K \cup \beta_{2}$ is therefore bounded vertically, unbounded horizontally, and separates $\mathbf{R}^{2}$. The complement of $\beta_{1} \cup K \cup \beta_{2}$ has exactly one connected component $V$ unbounded from above and bounded from below. By definition, the point $\widehat{y} \in\left(\ell_{1}, \ell_{2}\right)$ is above $K$, and as $\left(\beta_{1} \cup \beta_{2}\right) \cap\left(\ell_{1}, \ell_{2}\right)=\emptyset$, we have that $y \in V$.

By the periodicity of $\widehat{f}, \widehat{f}^{n_{1}}(V)$ is also unbounded from above and bounded from below. By construction of $\beta_{1}$ and $\beta_{2}$ we have that $\widehat{f}^{n_{1}}\left(\beta_{1}\right) \subset L\left(\ell_{1}\right)$ and $\widehat{f}^{n_{1}}\left(\beta_{2}\right) \subset R\left(\ell_{2}\right)$, that is

$$
\widehat{f}^{n_{3}}\left(\beta_{1} \cup \beta_{2}\right) \cap\left(\ell_{1}, \ell_{2}\right)=\emptyset .
$$

As $\widehat{f}^{n_{1}}(y) \in\left(\ell_{1}, \ell_{2}\right)$ is below $\widehat{f}^{n_{1}}(K)$ and as $\widehat{f}^{n_{1}}\left(\beta_{1} \cup \beta_{2}\right) \cap\left(\ell_{1}, \ell_{2}\right)=\emptyset$, we have that $\widehat{f}^{n_{1}}(y)$ belongs to a connected component of $\mathbf{R}^{2} \backslash \widehat{f}^{n_{1}}\left(\beta_{1} \cup K \cup \beta_{2}\right)$ which is unbounded from below. This contradicts the fact that $\widehat{f}^{n_{1}}(y)$ belongs to the set $\widehat{f}^{n_{1}}(V)$, which is bounded from below, and this contradiction finishes the proof of the lemma.

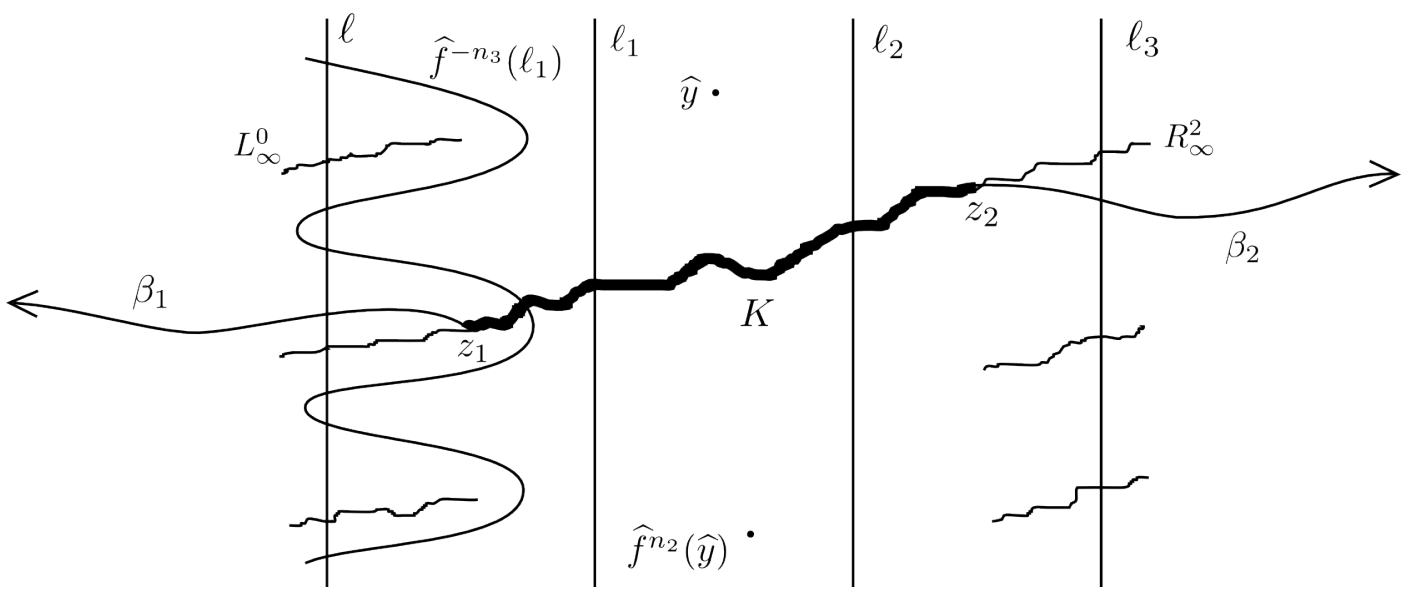

Figure 7: Proof of Lemma 4.21.

We end up this section with the proof of Lemma 4.19

Proof of Lemma 4.19 Let $p \in[a, b]$. By Proposition 4.17 and by the periodicity of $\widehat{f}$, there exists a closed $\widehat{f}$-invariant set $K \subset\left(\ell_{1}, \ell_{2}\right)$ such that $\rho(K, \hat{f})=\{(0, p)\}$. Observe that as $K \subset\left(\ell_{1}, \ell_{2}\right)$ is $\widehat{f}$-invariant, $K \subset L_{\infty}^{1} \cap R_{\infty}^{1}$.

Let $z \in K$, and let $E$ be the connected component of $L_{\infty}^{1} \cap R(\ell)$ that contains $z$. By Lemma 2.11 we have that

$$
\bar{E} \text { is compact, } \rho(\bar{E}, \widehat{f})=\{(0, p)\} \text {, and } \bar{E} \cap \ell \neq \emptyset \text {. }
$$

The sought set $C$ will be constructed from $E$. The construction is divided in the following cases.

Case 1: $\bar{E} \cap L_{\infty}^{0}=\emptyset$. 
In this case, $\widehat{f}^{n}(\bar{E}) \subset R\left(\ell_{1}\right)$ for some $n>0$, by the definition of $L_{\infty}^{0}$ and by the compacity of $\bar{E}$. Letting $x=z$, by (27) we have that $x$ satisfies items (1) to (4) of the lemma, with $C=E$.

Case 2: $\bar{E} \cap L_{\infty}^{0} \neq \emptyset$.

We divide this case in two subcases.

Case 2.1: For every connected component $B$ of $L_{\infty}^{0} \cap \bar{R}(\ell)$ that intersects $\bar{E}$, it holds $B \cap R_{\infty}^{0}=\emptyset$ (cr. Fig. 8).

In this case, for any such component $B$ there is $m_{B}>0$ such that $\widehat{f}^{-m_{B}}(B) \subset L(\ell)$. Then, by the compacity of $L_{\infty}^{0} \cap \bar{E}$, there is $m>0$ such that

$$
\widehat{f}^{-m}(\bar{E}) \cap\left(L_{\infty}^{0} \cap \bar{R}(\ell)\right)=\emptyset
$$

and therefore

$$
\overline{\widehat{f}-m(E) \cap R(\ell)} \cap L_{\infty}^{0}=\emptyset .
$$

Let $E_{0}$ be the connected component of $\widehat{f}^{-m}(E) \cap R(\ell)$ that contains $f^{-m}(z)$. Then $\bar{E}_{0}$ is compact because $\bar{E}$ is, and by 28 we have

$$
\bar{E}_{0} \cap L_{\infty}^{0}=\emptyset,
$$

so there is $n>0$ such that $f^{n}\left(\bar{E}_{0}\right) \subset R\left(\ell_{1}\right)$. By Lemma $2.11 \bar{E}_{0} \cap \ell \neq \emptyset$ and $\rho\left(\bar{E}_{0}, \widehat{f}\right)=\{(0, p)\}$. Therefore, letting $x=\widehat{f}^{-m}(z)$, we have that $x$ satisfies the conclusions of the lemma, with $C=E_{0}$.

Case 2.2: There is a connected component $B_{0}$ of $L_{\infty}^{0} \cap \bar{R}(\ell)$ that intersects $\bar{E}$, and such that $B_{0} \cap R_{\infty}^{0} \neq \emptyset$ (see Fig. 8).

Let $E_{1}$ be the connected component of $L_{\infty}^{0} \cap R\left(\ell_{-1}\right)$ that contains $B_{0}$. We have three possibilities for $E_{1}$ :

(i) $\bar{E}_{1} \cap L_{\infty}^{-1}=\emptyset$.

(ii) $\bar{E}_{1} \cap L_{\infty}^{-1} \neq \emptyset$.

To deal with case (i), let $w \in B_{0} \cap R_{\infty}^{0} \subset L_{\infty}^{0} \cap R_{\infty}^{0}$. Proceeding as in Case 1 above, and using the periodicity of $\widehat{f}$ we have that the point $x=T_{1}(w) \in L_{\infty}^{1} \cap R_{\infty}^{1}$ satisfies the conclusions of the lemma, with $C=T_{1}\left(B_{0}\right)$.

We now deal with case (ii). Let $D$ be a connected component of $L_{\infty}^{-1} \cap \bar{R}\left(\ell_{-1}\right)$ that intersects $\bar{E}_{1}$. Recall that through all of Section refsec.ss we are assuming that there are $z \in \mathbf{R}^{2}, n \in \mathbf{N}$ such that $\operatorname{pr}_{1}\left(\widehat{f}^{n}(z)-z\right)>M$, and by the construction of $M, \widehat{f}^{n}\left(\ell_{-1}\right) \cap R\left(\ell_{2}\right) \neq \emptyset$ (cf. equation (5)). Then, by Lemma 2.11 and as $\bar{E}_{1} \cup D \cup E \subset L_{\infty}^{1}, \rho\left(\bar{E}_{1} \cup D \cup E, \widehat{f}\right)$ consists of a point. As $\rho(\bar{E}, \widehat{f})=\{(0, p)\}$, we then have

$$
\rho\left(\bar{E}_{1} \cup D \cup \bar{E}, \widehat{f}\right)=\{(0, p)\} .
$$

The set $\bar{E}_{1} \cup D \cup \bar{E}$ is compact, intersects $L_{\infty}^{-1}$ (because $D$ does), and intersects also $R_{\infty}^{1}$ (as $z \in E$ ). This, together with (29), contradicts Lemma 4.21. Therefore, case (ii) cannot occur, and this finishes the proof of the lemma. 

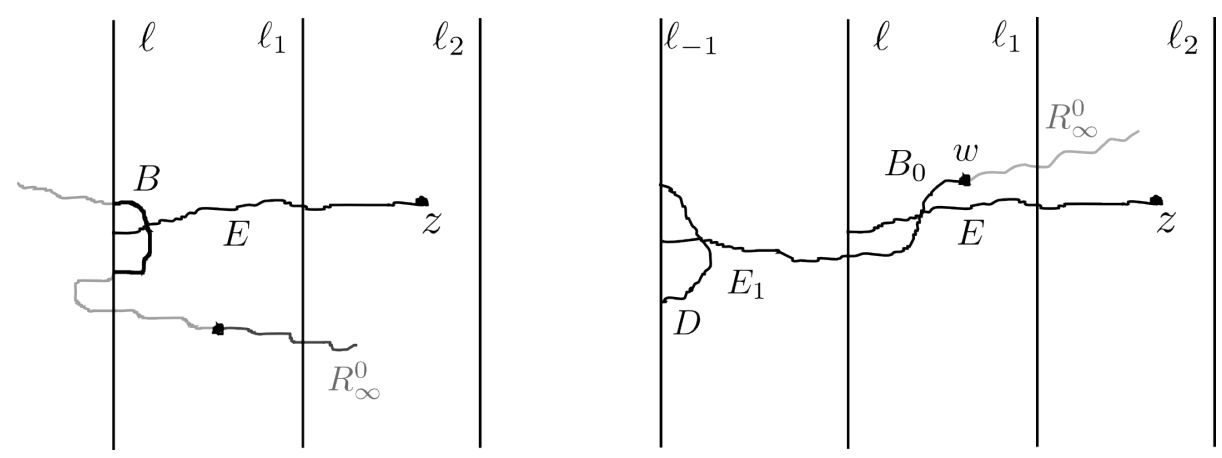

Figure 8: Proof of Lemma 4.19, Left: Case 2.1. Right: Case 2.2-(ii).

\subsubsection{Definition of the sets $L_{i}, R_{i}$ and $\mathcal{V}$.}

We are now ready to define the sets $L_{i}, R_{i}, \mathcal{V}$ and verify that they satisfy Lemma 4.13 The sets $L_{i}$ and $R_{i}$. By Corollary 4.20 , there exists an $\operatorname{arc} I \subset \ell$, and two points $x, y \in L_{\infty}^{1} \cap R_{\infty}^{1}$ such that the connected components $L_{x}$ and $L_{y}$ of $L_{\infty}^{1} \cap R(\ell)$ that contain $x$ and $y$, respectively, are such that:

- $\bar{L}_{x}$ and $\bar{L}_{y}$ are compact,

- $\bar{L}_{i} \cap I \neq \emptyset$, for $i=x, y$,

- $\bar{L}_{i} \cap L_{\infty}^{0}=\emptyset$, for $i=x, y$,

- $\rho\left(\bar{L}_{i}, \widehat{f}\right)=\left\{\left(0, r_{i}\right)\right\}$, for $i=x, y$ and some reals $r_{x} \neq r_{y}$,

- $\operatorname{int}(I) \cap L_{\infty}^{0}=\emptyset$, and the endpoints of $I$ lie in $L_{\infty}^{0}$.

Define $L_{1}$ as a connected component of $L_{x} \backslash R_{\infty}^{1}$ such that $\bar{L}_{1} \cap I \neq \emptyset$, and similarily, define $L_{2}$ as a connected component of $L_{y} \backslash R_{\infty}^{1}$ such that $\bar{L}_{2} \cap I \neq \emptyset$. By the above properties, we have $L_{i} \subset L_{\infty}^{1} \backslash L_{\infty}^{0}$ and $\rho\left(\bar{L}_{i}, \widehat{f}\right)=r_{i}, r_{1} \neq r_{2}$, and then the sets $L_{i}$ satisfy items $1 \mathrm{a}$ and $1 \mathrm{c}$ from Lemma 4.13 .

Define $R_{1}$ and $R_{2}$ to be connected components of $R_{\infty}^{1}$ that intersect $\bar{L}_{1}$ and $\bar{L}_{2}$, respectively 3 Then item $1 \mathrm{~b}$ from Lemma 4.13 holds.

As $\bar{L}_{i} \cap I \neq \emptyset$ and the $R_{i}$ are unbounded to the right (cf. Lemma 2.9p, the sets $F_{i}=L_{i} \cup R_{i}$ separate $R(\ell)$. By definition the $L_{i}$ and $R_{i}$ are connected, and by the above mentioned properties the $\bar{L}_{i}$ are compact. Thus, item $1 \mathrm{~d}$ from Lemma 4.13 holds.

The set $\mathcal{V}$. Item $2 \mathrm{a}$ from Lemma 4.13 states that $\mathcal{V}$ is of the form $\mathcal{V}=\left(V_{1} \cap V_{2}\right) \cup R_{1}$, for some open sets $V_{1}, V_{2} \subset R(\ell)$. We construct now such sets.

Claim 4.22. Let $i \in\{1,2\}$ and let $p, q \in \ell \backslash \bar{F}_{i}, x \in \ell \cap \bar{F}_{i}$ be points such that $p<x<q$, where $<$ denotes the order of $\ell$ induced by its upwards orientation. Then, $p$ and $q$ belong to different connected components of $\bar{R}(\ell) \backslash \bar{F}_{i}$.

Proof. This follows easily from the fact that $F_{i}$ is connected and separates $R(\ell)$.

\footnotetext{
${ }^{3}$ Note that, in principle, the set $R_{\infty}^{1}$ might be connected, and in such case $R_{1}=R_{2}$.
} 
A consequence of Claim 4.22 is that, as the sets $F_{i}$ separate $R(\ell)$ and as $\bar{L}_{1} \cap \bar{L}_{2}=\emptyset$, either $\min \bar{L}_{1} \cap \ell>\max \bar{L}_{2} \cap \ell$ or $\min L_{2} \cap \ell>\max \bar{L}_{1} \cap \ell$. Without loss of generality, suppose it holds the former;

$$
\min \bar{L}_{1} \cap \ell>\max \bar{L}_{2} \cap \ell,
$$

that is, in some sense $L_{1}$ is 'above' $L_{2}$.

As $\bar{L}_{1} \cap \ell$ is compact (because $\bar{L}_{1}$ is), and as $R_{1} \subset R_{\infty}^{1} \subset R\left(\ell_{1}\right)$, we have that $\bar{F}_{1} \cap \ell$ is compact, and therefore there is only one connected component $V_{1}$ of $R(\ell) \backslash F_{1}$ whose closure contains a subcurve of $\ell$ that is unbounded from below. Analogously, there is only one connected component $V_{2}$ of $R(\ell) \backslash F_{2}$ whose closure contains a subcurve of $\ell$ that is unbounded from above (see Fig. 5).

Note that, by definition, $\partial V_{i} \subset F_{i} \cup \ell$, for $i=1,2$. Therefore, to show that the set

$$
\mathcal{V}:=\left(V_{1} \cap V_{2}\right) \cup R_{1}
$$

satisfies item 2a from Lemma 4.13 , it suffices to show the following:

Claim 4.23. $L_{1} \subset V_{2}$, and $L_{2} \subset V_{1}$.

Proof of Claim 4.23. We will prove that $L_{1} \subset V_{2}$, the proof that $L_{2} \subset V_{1}$ being symmetric. First observe that by $(30)$ and by definition of $V_{2}$, it follows that $L_{1} \cap V_{2} \neq \emptyset$. As $L_{1}$ is by definition connected and disjoint from $R_{2}$, it holds

$$
L_{1} \subset V_{2} .
$$

Note that by $(30)$ and by definition of $V_{1}, V_{2}$, it follows that $\emptyset \neq V_{1} \cap V_{2} \subset \operatorname{int}(\mathcal{V}), \overline{\mathcal{V}} \cap \ell \neq \emptyset$ and as $\mathcal{V}$ is unbounded to the right, $\mathcal{V}$ separates $R(\ell)$. Also by definition, $\partial \mathcal{V} \subset F_{1} \cup F_{2} \cup \ell$, and therefore item $2 \mathrm{~b}$ from Lemma 4.13 holds.

As the sets $F_{i}$ separate $R(\ell)$ and as $\bar{F}_{i} \cap\left[\ell, \ell_{1}\right]=\bar{L}_{i} \cap\left[\ell, \ell_{1}\right]$ is compact, we have that $\bar{V}_{1} \cap\left[\ell, \ell_{1}\right]$ is bounded from above and $\bar{V}_{2} \cap\left[\ell, \ell_{1}\right]$ is bounded from below. Therefore $\overline{\mathcal{V}} \cap\left[\ell, \ell_{1}\right] \subset$ $\left(\bar{V}_{1} \cap \bar{V}_{2}\right) \cap\left[\ell, \ell_{1}\right]$ is compact, which proves item $2 \mathrm{~d}$

Observe that, also by (30) and by definition of $\mathcal{V}$, the arc $I$ mentioned above and given by Corollary 4.20 contains a non-degenerate arc $J$ such that $J=\partial \mathcal{V} \cap \ell$, and such that $J(0) \in \bar{L}_{2}$, $J(1) \in \bar{L}_{1}$ and $J \cap L_{\infty}^{0}=\emptyset$. Thus, item $2 \mathrm{e}$ holds.

By last, we prove item 2c, namely, there is $n_{1}>0$ such that $\widehat{f}^{n_{1}}(\mathcal{V}) \subset R\left(\ell_{1}\right)$. To this end, it suffices to show that $\overline{\mathcal{V}} \cap L_{\infty}^{0}=\emptyset$. Suppose this is not the case, and let $x \in \overline{\mathcal{V}} \cap L_{\infty}^{0}$. Consider the connected component $C$ of $L_{\infty}^{0}$ that contains $x$. As $\partial \mathcal{V} \subset J \cup F_{1} \cup F_{2}$, and as the sets $J, F_{1}, F_{2}$ do not intersect $L_{\infty}^{0}$, we then have that $C$ is contained in $\operatorname{int}(\mathcal{V}) \subset R(\ell)$. This contradicts the fact that $C$ is unbounded to the left (Lemma 2.11). We therefore must have $\overline{\mathcal{V}} \cap L_{\infty}^{0}=\emptyset$, and item 2c holds.

\subsection{Case that $\mathcal{C}$ contains singularities}

As we did in the case that $\mathcal{C}$ does not contain singularities, we will prove that if $M=M(f)>0$ is the constant constructed in Section 4.3 and if there are $x \in \mathbf{R}^{2}$ and $N_{1}>0$ such that $\operatorname{pr}_{1}\left(\widehat{f}^{N_{1}}(x)-x\right)>M$, then there are $z \in \mathbf{R}^{2}$ and $N>0$ such that $\operatorname{pr}_{1}\left(\widehat{f}^{n N}(z)-z\right)>n$ for all $n \in \mathbf{N}$.

We begin by giving an idea of the proof. 


\subsubsection{Idea of the proof}

As we mentioned, we are assuming that there is $x \in \mathbf{R}^{2}$ and $N_{1}>0$ such that $\operatorname{pr}_{1}\left(\widehat{f}^{N_{1}}(x)-x\right)>$ $M$. Recall from 10 that this implies $\widehat{f}^{N_{1}}\left(\mathcal{C}_{i}\right) \cap \mathcal{C}_{i+2} \neq \emptyset$ for any $i$, and in particular there are leaves $\gamma \subset \mathcal{C}_{-2}$ and $\beta \subset \mathcal{C}_{0}$ such that $\widehat{f}^{N_{1}} \gamma \cap \beta \neq \emptyset$. Recall also from $(6)$ that there is $p \in \mathbf{R}^{2}$ such that $\pi(p)$ is periodic for $f$ and $\rho(\pi(p), \widehat{f})=(0, c)$, for some $c<0$. We will first choose an integer translate of $p$, denoted also $p$, such that $p \in R\left(\mathcal{C}_{-2}\right) \cap L\left(\mathcal{C}_{0}\right)$ and $p$ is above $\widehat{f}^{N_{1}}(\gamma)$.

We will show that, as the isotopy $\left(f_{t}\right)_{t}$ is positively transverse to the foliation $\widehat{\mathcal{F}}$, all the iterates $\widehat{f}^{N_{1}+n}(\gamma), n>0$, also intersect $\beta$, which might be interpreted as $\widehat{f}^{N_{1}}(\gamma)$ being 'anchored' to $\beta$.

We will choose an integer translation $T: \mathbf{R}^{2} \rightarrow \mathbf{R}^{2}$ such that $T(\beta) \subset \mathcal{C}_{1}$ and $T(\beta)$ is below $\beta$. Observe that by the periodicity of $\widehat{f}, \widehat{f}^{-N_{1}}(T(\beta)) \cap \mathcal{C}_{-1} \neq \emptyset$. We will then choose $N_{2}>0$ such that $\widehat{f}^{N_{2}}(p)$ is below $\widehat{f}^{-N_{1}}(T(\beta))$ (see Fig. 9 ). As $\widehat{f}^{N_{1}} \gamma$ is 'anchored' to $\beta$, the iterates of $p$ by $\widehat{f}$ will 'push' the curve $\widehat{f}^{N_{1}} \gamma$ downwards, and we will show that

$$
\widehat{f}^{N_{1}+N_{2}} \gamma \cap \widehat{f}^{-N_{1}}(T(\beta)) \neq \emptyset,
$$

which in turn implies

$$
\widehat{f}^{2 N_{1}+N_{2}} \gamma \cap \mathcal{C}_{1} \supset \widehat{f}^{2 N_{1}+N_{2}} \gamma \cap T(\beta) \neq \emptyset .
$$

We want to continue this process inductively, in order to prove the following:

Lemma 4.24. $\widehat{f}^{(i+1) N_{1}+i N_{2}} \gamma \cap \mathcal{C}_{i} \neq \emptyset \quad \forall i \in \mathbf{N}$.

This lemma clearly implies that $\max \left(\operatorname{pr}_{1}(\rho(\widehat{f}))\right)>0$. This in turn implies that $\operatorname{int}(\rho(\widehat{f})) \neq \emptyset$, and then by Theorem 2.3 we have that there is a periodic point $q$ for $f$ such that $\operatorname{pr}_{1}(\rho(q, \widehat{f}))>0$. This will yield Theorem D.

Let us explain the main ideas in Lemma 4.24 As we saw, we have $\widehat{f}^{2 N_{1}+N_{2}} \gamma \cap T(\beta) \neq \emptyset$. The first main step in the proof will be to show that the iterate $\widehat{f}^{2 N_{1}+N_{2}} \gamma$ is well positioned with respect to the point $T(p)$ (cf. Definition 4.29), which very roughly speaking means that $\widehat{f}^{2 N_{1}+N_{2}} \gamma$ is in some sense 'below' $T(p)$. Also, the fact that $\left(f_{t}\right)_{t}$ is positively transverse to $\widehat{\mathcal{F}}$ will imply that $\widehat{f}^{2 N_{1}+N_{2}+n} \gamma \cap T(\beta) \neq \emptyset$ for all $n \geq 0$, and then $\widehat{f}^{2 N_{1}+N_{2}} \gamma$ is 'anchored' to $T(\beta)$. Therefore, the forward iterates of $T(p)$ by $\widehat{f}$ will 'push' the arc $\widehat{f}^{2 N_{1}+N_{2}} \gamma$ downwards, and we will show that

$$
\widehat{f}^{2 N_{1}+2 N_{2}} \gamma \cap \widehat{f}^{-N_{1}}\left(T^{2}(\beta)\right) \neq \emptyset .
$$

The second main step in the proof will be to show that this intersection is a good intersection, meaning that $\widehat{f}^{2 N_{1}+2 N_{2}} \gamma$ intersects $\widehat{f}^{-N_{1}}\left(T^{2}(\beta)\right)$ in a way such that $\widehat{f}^{3 N_{1}+2 N_{2}} \gamma$ is well positioned with respect to $T^{2}(p)$.

In this way, by induction we will obtain Lemma 4.24 , which as we mentioned, implies Theorem D.

The precise definitions of being well positioned and having good intersection are given in Section 4.6.3. To introduce such concepts, some previous definitions are given in 4.6.2. The induction steps for the proof of Lemma 4.24 are stated in Section 4.6.4, and in 4.6.5 it is shown how the lemma follows from them. Finally, the proof of the induction steps is carried out in sections 4.6 .6 and 4.6 .7

\subsubsection{The points $p_{i}$, the $\operatorname{arcs} \lambda, \widetilde{\gamma}$, the leaves $\beta_{i}$ and the integer $N_{2}$.}

In this section we give some definitions that will be used through the rest of 4.6 . 


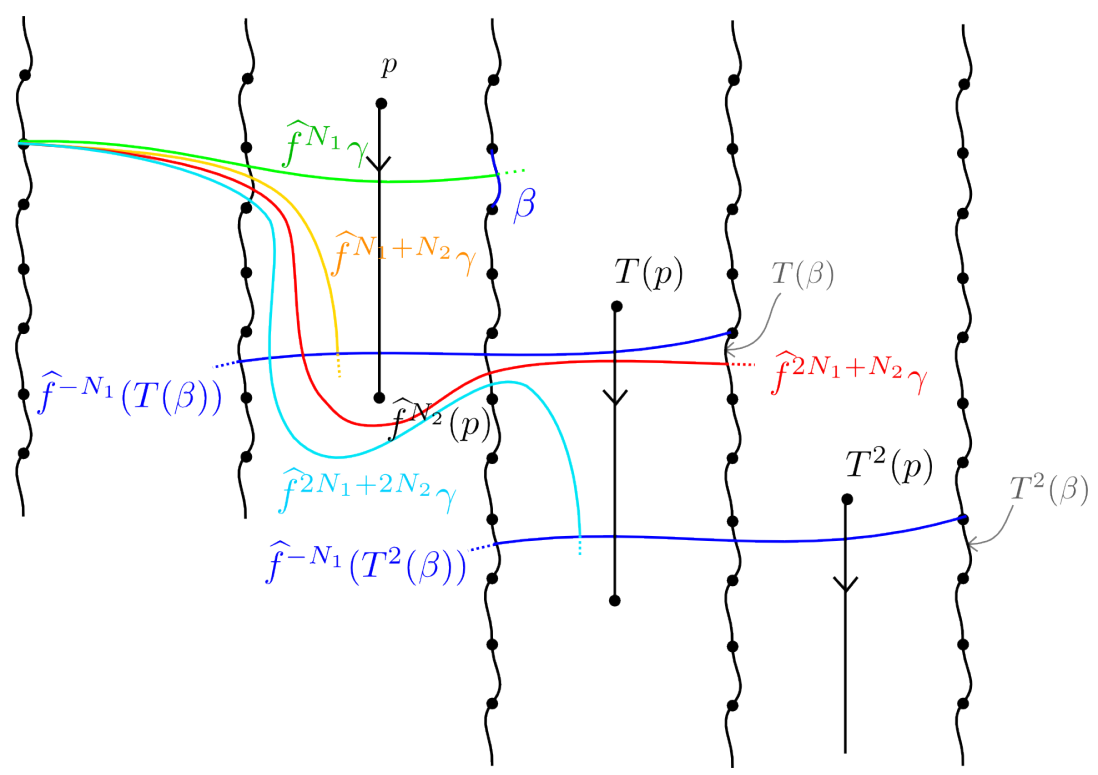

Figure 9:

The arc $\lambda$ and the leaf $\beta$. Recall that $\widehat{f}^{N_{1}}\left(\mathcal{C}_{i}\right) \cap \mathcal{C}_{i+2} \neq \emptyset$ for all $i$ (see $10 p$ ), and in particular $\widehat{f}^{N_{1}}\left(\mathcal{C}_{-2}\right) \cap \mathcal{C}_{0} \neq \emptyset$. Let $\gamma$ be a leaf of $\widehat{\mathcal{F}}$ contained in $\mathcal{C}_{-2}$ such that

$$
\widehat{f}^{N_{1}}(\gamma) \cap \mathcal{C}_{0} \neq \emptyset
$$

We will now chose a leaf $\beta$ of $\widehat{\mathcal{F}}$ contained in $\mathcal{C}_{0}$ such that $\widehat{f}^{N_{1}}(\gamma) \cap \beta \neq \emptyset$, and an arc $\lambda$ going from $\operatorname{sing}(\gamma)$ to $\widehat{f}^{N_{1}}(\gamma) \cap \beta$. Such choice will be done separately for two possibilities on $\gamma$ :

Case 1: Either $\alpha(\gamma)$ or $\omega(\gamma)$ consist of a singularity.

If $\omega(\gamma)$ consists of a singularity (see Fig. 10 , define $\beta$ as the leaf that contains the last point of intersection of $\widehat{f}^{N_{1}} \gamma$ with $\mathcal{C}_{0}$, that is, $\beta$ is the leaf of $\widehat{\mathcal{F}}$ that contains $\gamma\left(t_{*}\right)$, where

$$
t_{*}=\max \left\{t \in(0,1): \widehat{f}^{N_{1}} \gamma(t) \in \mathcal{C}_{0}\right\} .
$$

Define then the arc

$$
\lambda=\left(\left.\widehat{f}^{N_{1}} \gamma\right|_{\left[t_{*}, 1\right]}\right)^{-1}
$$

Analogously, if $\omega(\gamma)$ does not consist of a singularity but $\alpha(\gamma)$ does, define $\beta$ as the first point of intersection of $\widehat{f}^{N_{1}}(\gamma)$ with $\mathcal{C}_{0}$; that is, let $\beta$ be the leaf of $\widehat{\mathcal{F}}$ that contains $\gamma\left(t_{*}\right)$, where

$$
t_{*}=\min \left\{t \in(0,1): \widehat{f}^{N_{1}} \gamma(t) \in \mathcal{C}_{0}\right\}
$$

and define

$$
\lambda=\left.\widehat{f}^{N_{1}} \gamma\right|_{\left[0, t_{*}\right]} .
$$

Note that, in both cases, $\lambda(0) \in \operatorname{sing}(\gamma),\left.\lambda\right|_{[0,1)} \subset L\left(\mathcal{C}_{0}\right)$ and $\lambda(1) \in \beta \subset \mathcal{C}_{0}$.

Case 2: Neither $\alpha(\gamma)$ or $\omega(\gamma)$ consist of a singularity.

Note that by Corollary 4.9 we have that $\alpha(\gamma)$ and $\omega(\gamma)$ are compact, and then by Theorem 2.22 we have in this case that $\alpha(\gamma)$ and $\omega(\gamma)$ are either loops or generalized cycles of connections. 
Thus, there may not exist neither a first or a last point of intersection of $\widehat{f}^{N_{1}}(\gamma)$ with $\mathcal{C}_{0}$ (see Fig. 10). We will define the auxiliary $\operatorname{arc} \lambda$, and then we will define $\beta$ as the leaf of $\widehat{\mathcal{F}}$ containing $\lambda(1)$.

Let $s$ be any singularity contained in Fill $(\omega(\gamma))$, such that there is no other singularity $s^{\prime} \in$ Fill $(\omega(\gamma))$ with $\operatorname{pr}_{2}\left(s^{\prime}\right)=\operatorname{pr}_{2}(s)$ and $\operatorname{pr}_{1}\left(s^{\prime}\right)<\operatorname{pr}_{1}(s)$. Let $\lambda_{1}$ be the straight horizontal arc going leftwards from $s$ to a point of $\widehat{f}^{N_{1}}(\gamma)$ sufficiently close to $\widehat{f}^{N_{1}}(\omega(\gamma))$ so that $\widehat{f}^{-N_{1}}\left(\lambda_{1}\right) \cap \mathcal{C}_{0}=\emptyset$. Let $\lambda_{2}$ be a subarc of $\widehat{f}^{N_{1}} \gamma$ going from $\lambda_{1}(1)$ to a point $z \in \widehat{f}^{N_{1}} \gamma \cap \mathcal{C}_{0}$ such that $\left.\lambda_{2}\right|_{[0,1)} \subset L\left(\mathcal{C}_{0}\right)$.

Let then $\lambda=\lambda_{1} \cdot \lambda_{2}$, and define $\beta$ as the leaf of $\widehat{\mathcal{F}}$ that contains $\lambda(1)$.

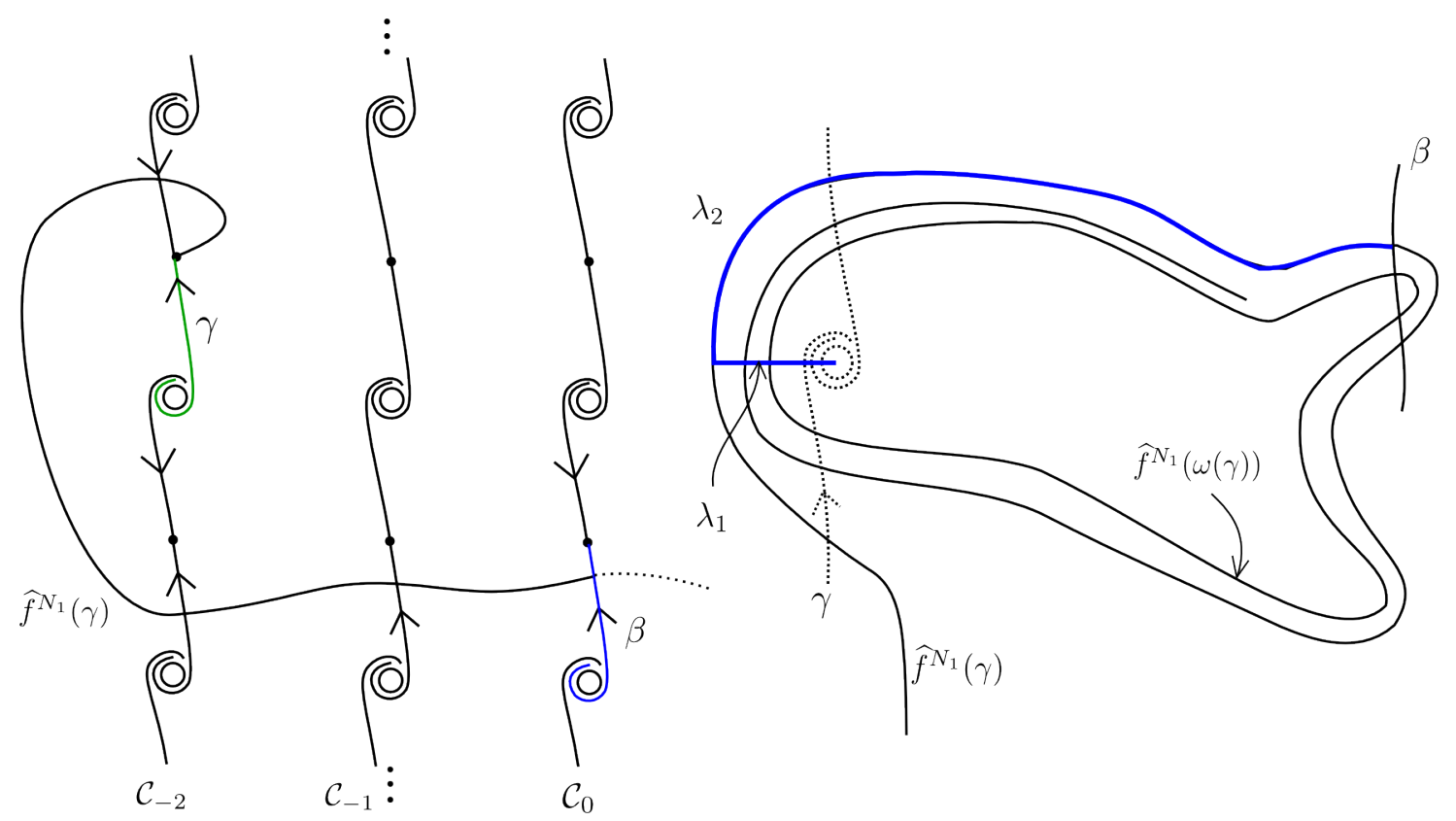

Figure 10: Definition of $\beta$. Left: case that $\omega(\gamma)$ consists of a singularity. Right: case that neither $\omega(\gamma)$ nor $\alpha(\gamma)$ consist of a singularity.

The points $p_{i}$ and the leaves $\beta_{i}$. Recall from (6) and (7) that there is $p \in \mathbf{R}^{2}$ such that $\pi(p) \in \mathbf{T}^{2}$ is periodic and rotates downwards, $l_{1}, l_{2}$ are straight vertical lines oriented upwards such that $\mathcal{C}_{0} \subset L\left(l_{1}\right)$ and $R\left(l_{1}\right) \subset\left\{\widehat{f}_{t}(p): t \in \mathbf{R}\right\} \subset L\left(l_{2}\right)$, and $n_{1} \in \mathbf{N}$ is such that $\mathcal{C}_{1}=$ $T_{1}^{n_{1}}\left(\mathcal{C}_{0}\right) \subset R\left(l_{2}\right)$. Fix an integer translate $p_{0}$ of $p$ of the form $p_{0}=T_{2}^{i} T_{1}^{-n_{1}} \in R\left(\mathcal{C}_{-1}\right) \cap L\left(\mathcal{C}_{0}\right)$, with $i$ such that:

- $p_{0}$ is above $\lambda$, and

- $\left\{\widehat{f}_{t}\left(p_{0}\right): t \in(-\infty, 0]\right\}$ is above $\left\{\widehat{f}_{t}(\beta): t \in\left[-N_{1}, 0\right]\right\}$.

Note that the second condition on $p_{0}$ may indeed be satisfised, because $\pi\left(p_{0}\right) \in \mathbf{T}^{2}$ is periodic and rotates downwards.

Define then

$$
\ell_{1}=T_{1}^{-n_{1}} l_{1}, \quad \ell_{2}=T_{1}^{-n_{1}} l_{2}
$$

and note that

$$
\left\{\widehat{f}_{t}\left(p_{0}\right): t \in \mathbf{R}\right\} \subset\left(\ell_{1}, \ell_{2}\right) \subset R\left(\mathcal{C}_{-1}\right) \cap L\left(\mathcal{C}_{0}\right) .
$$


Section 4.7 will be devoted to the proof of the following.

Lemma 4.25. There exists an arc $\delta$ such that (see Fig. 11):

1. $\operatorname{int}(\delta) \subset \widehat{f}^{-N_{1}} \beta$,

2. $\delta(0) \in \mathcal{C}_{-2}, \delta(1) \in \mathcal{C}_{0}, \operatorname{int}(\delta) \subset R\left(\mathcal{C}_{-2}\right) \cap L\left(\mathcal{C}_{0}\right)$,

3. $\delta$ leaves a leaf $\delta_{1} \subset \mathcal{C}_{-2}$ on $t=0$ by the right (cf. Definition 2.18),

4. one of the following holds:

(a) $\delta(1) \in \operatorname{sing}(\widehat{\mathcal{F}}) \cap \mathcal{C}_{0}$.

(b) $\delta$ arrives in a leaf $\delta_{2} \subset \mathcal{C}_{0}$ on $t=1$ by the right.

Notation 4.26. For not having to continuously refer to $\delta$ separately for cases $4(a)$ and $4(b)$ from last lemma, we define $\widetilde{\delta}_{2}=\emptyset$ in case item $4(a)$ holds, and $\widetilde{\delta}_{2}=\delta_{2}$ in case item $4(b)$ holds.

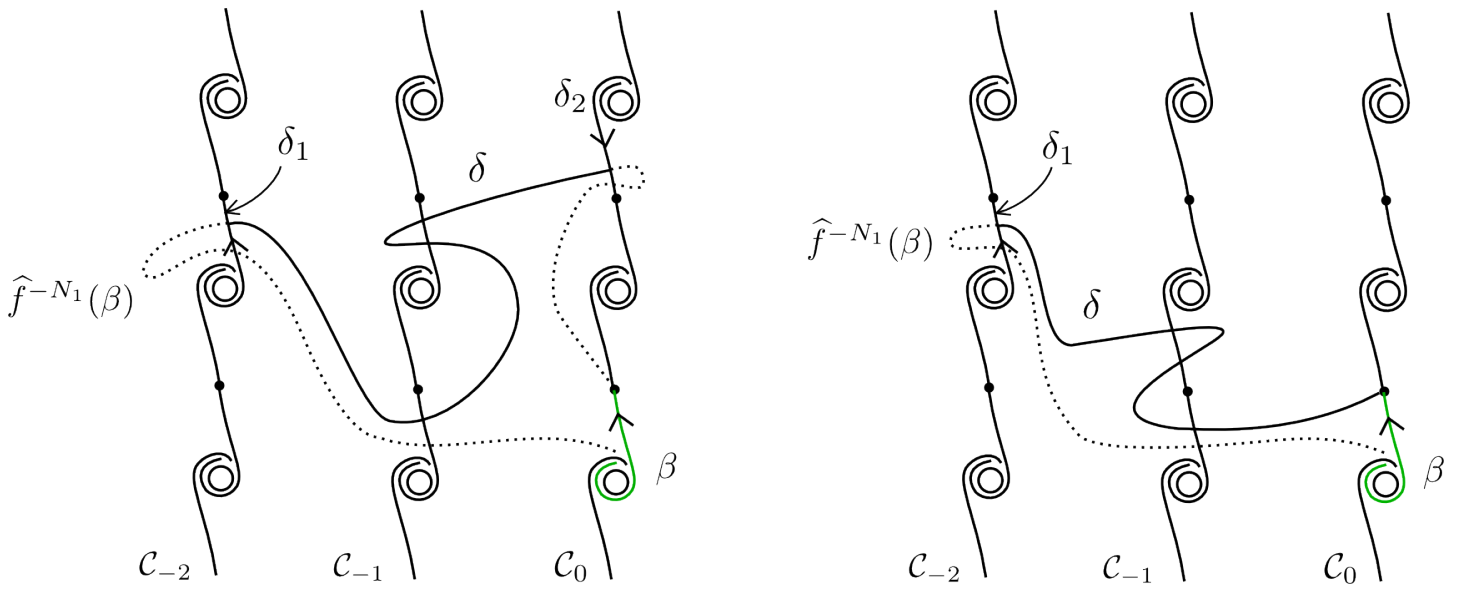

Figure 11: The arc $\delta \subset \widehat{f}^{-N_{1}}(\beta)$. Left: case that $\delta(1)$ belongs to a leaf $\delta_{2} \subset \mathcal{C}_{0}$. Right: case that $\delta(1)$ is a singularity.

Define $n_{2}>0$ such that

$\widehat{f}^{-N_{1}} \lambda \cup \lambda \cup\left\{\widehat{f}_{t}(\beta): t \in\left[-N_{1}, 0\right]\right\}$ is above $T_{1}^{n_{1}} T_{2}^{-n_{2}}\left(\left\{\widehat{f}_{t}(\beta): t \in\left[-N_{1}, 0\right]\right\} \cup \delta_{1} \cup \widetilde{\delta}_{2}\right)$.

Denote

$$
T=T_{1}^{n_{1}} T_{2}^{-n_{2}},
$$

and for $i \geq 0$ let

$$
\beta_{i}=T^{i}(\beta) \quad \text { and } \quad p_{i}=T^{i}\left(p_{0}\right),
$$

(see Fig. 12). Note that by 31 , the paths $\left\{\widehat{f}_{t}\left(p_{i}\right)\right\}$ are disjoint.

The integer $N_{2}$. The definition of $N_{2}$ is given by the following.

Claim 4.27. There exists $N_{2}>0$ such that: 


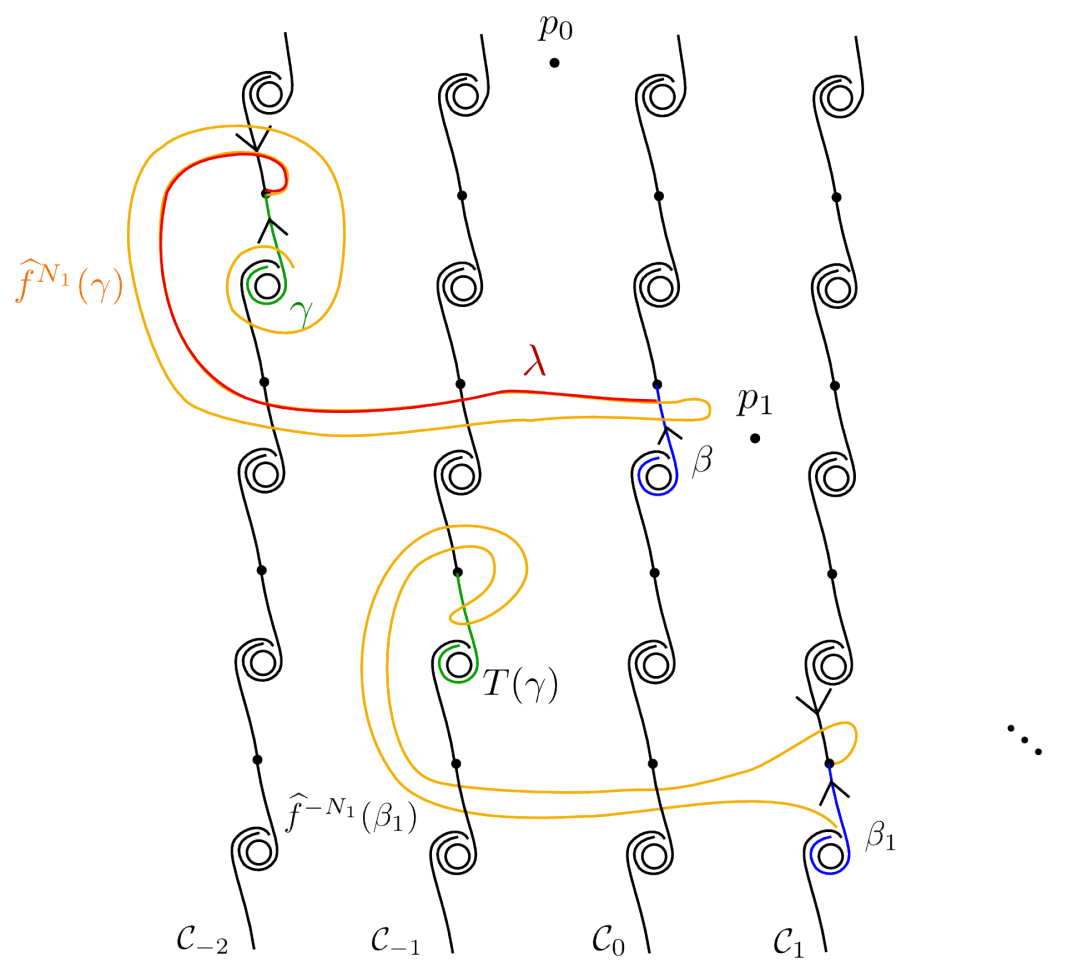

Figure 12: $\widehat{f}^{-N_{1}} \beta_{1}$ is below $\lambda$, and $p_{0}$ is above $\lambda$.

1. $\widehat{f}^{N_{2}}\left(p_{0}\right)$ is below $\widehat{f}^{-N_{1}}\left(\beta_{1}\right)$, and

2. $\widehat{f}^{-k\left(N_{1}+N_{2}\right)}\left(p_{k}\right)$ is above $p_{0}$ for all $k \in \mathbf{N}$.

Proof. Denote by $\pi: \mathbf{R}^{2} \rightarrow \mathbf{T}^{2}$ the canonical projection. As $\pi\left(p_{0}\right) \in \mathbf{T}^{2}$ is periodic and rotates downwards, and as $\widehat{f}^{-N_{1}}\left(\beta_{1}\right)$ is bounded (because by $\beta$ is bounded by Corollary 4.9 the existence of $N_{2}^{1}>0$ satisfying Item 1 is trivial. Now we see that there is $N_{2}^{2}>0$ satisfying Item 2. Taking $N_{2}=\max \left\{N_{1}^{1}, N_{2}^{2}\right\}$, this will prove the claim.

Also by the fact that $\pi\left(p_{0}\right) \in \mathbf{T}^{2}$ is periodic and rotates downwards, there is $N>0$ such that $\widehat{f}^{-n}\left(p_{1}\right)$ is above $p_{0}$, for all $n \geq N$. By induction this gives us that $\widehat{f}^{-k n}\left(p_{k}\right)$ is above $p_{0}$ for all $n \geq N$ and $k \in \mathbf{N}$. Choosing $N_{2}^{2}=N$, we get in particular that $\widehat{f}^{-k\left(N_{1}+N_{2}^{2}\right)}\left(p_{k}\right)$ is above $p_{0}$ for all $k \in \mathbf{N}$. That is, $N_{2}^{2}$ satisfies Item 2 , as desired.

Remark 4.28. Taking $N_{2}$ as a multiple of the period of $\pi\left(p_{0}\right)$ for $f$, we may assume that $\operatorname{pr}_{1}\left(\widehat{f}^{N_{2}}\left(p_{i}\right)\right)=\operatorname{pr}_{1}\left(p_{i}\right)$, for all $i$.

The arc $\tilde{\gamma}$. By last, we define $\tilde{\gamma}$ as

$$
\widetilde{\gamma}=\widehat{f}^{-N_{1}} \lambda \text {. }
$$

Observe that, as $\widehat{f}^{N_{1}}(\widetilde{\gamma})=\lambda$, by the definition of $p_{0}$,

$$
\widehat{f}^{N_{1}} \tilde{\gamma} \text { is below } p_{0},
$$

and by 32

$$
\widehat{f}^{N_{1}} \tilde{\gamma} \quad \text { is above } \widehat{f}^{-N_{1}}(T(\beta)) \text {. }
$$


Note also that for all $n, \widehat{f}^{n} \tilde{\gamma}(0)=\widehat{f}^{n-N_{1}} \lambda(0)=\lambda(0) \in \operatorname{sing}(\widehat{\mathcal{F}}) \cap \mathcal{C}_{-2}$, and $\widehat{f}^{N_{1}} \tilde{\gamma}(1)=\lambda(1) \in$ $\beta \subset \mathcal{C}_{0}$.

\subsection{3 'Well positioned' and 'good intersection'.}

Recall that for a bounded leaf $\ell \in \widehat{\mathcal{F}}, \operatorname{sing}(\ell)$ denotes the set of singularities of $\widehat{\mathcal{F}}$ contained in Fill $(\alpha(\ell)) \cup$ Fill $(\omega(\ell))$. Also, recall that by Corollary 4.9 every leaf $\ell$ of $\widehat{\mathcal{F}}$ contained in $\cup_{i} \mathcal{C}_{i}$ is bounded, and therefore for such $\ell, \operatorname{sing}(\ell) \neq \emptyset$.

Let $\delta$ be the arc given by Lemma 4.25 For each $n \in \mathbf{N}_{0}$ we now define

$F_{n}=\left\{\widehat{f}^{-k\left(N_{1}+N_{2}\right)}\left(p_{n+k}\right): k \in \mathbf{N}_{0}\right\} \bigcup \cup_{k \in \mathbf{N}_{0}} \operatorname{sing}\left(\beta_{n+k}\right) \bigcup \cup_{k \in \mathbf{N}_{0}} T^{k}\left(\operatorname{sing}\left(\delta_{1}\right) \cup \operatorname{sing}\left(\widetilde{\delta}_{2}\right)\right)$,

and proceed to the definitions of 'well positioned' and 'good intersection'. Consider the arc $\widetilde{\gamma}$ defined in 4.6 .2

Definition 4.29. Let $i \in \mathbf{N}_{0}$. We say that an iterate $\widehat{f}^{n}(\tilde{\gamma})$ of $\tilde{\gamma}$ is well positioned with respect to $p_{i}$ if $\widehat{f}^{n}(\tilde{\gamma})$ contains a subarc $\eta$ such that (see Fig. 13 ):

1. $\eta(0)=\widehat{f}^{n}(\tilde{\gamma}(0))=\tilde{\gamma}(0) \in \operatorname{sing}(\widehat{\mathcal{F}}) \cap \mathcal{C}_{-2}$,

2. $\eta(1) \in \beta_{i}$, and

3. $\eta$ is homotopic wfe $\operatorname{Rel}\left(F_{i}\right)$ to an arc $\kappa$ of the form $\kappa=\kappa_{1} \cdot \kappa_{2}$, where:

(a) $\kappa_{1}$ is a vertical arc from $\eta(0)$ to a point in the strip

$$
B_{i}=\left\{x \in \mathbf{R}^{2}: x \text { is below } p_{i} \text { and above } \beta_{i+1} \cup T^{i+1}\left(\delta_{1} \cup \widetilde{\delta}_{2}\right)\right\},
$$

and

(b) $\kappa_{2}$ is an arc contained in $B \backslash F_{i}$ from $\kappa_{1}(1)$ to $\eta(1) \in \beta_{i}$ such that, if $\bar{\kappa}_{2}$ is a lift of $\kappa_{2}$ to the universal cover of $\mathbf{R}^{2} \backslash\left(\operatorname{sing}(\widehat{\mathcal{F}}) \cap F_{i}\right)$, and if $\bar{\beta}$ is a lift of $\beta_{i}$ containing $\bar{\kappa}_{2}(1)$, then $\bar{\kappa}_{2}(0) \in L(\bar{\beta})$.

As we mentioned in $₫ 4.6 .1$, last definition gives us that $\widehat{f}^{n} \widetilde{\gamma}$ contains an arc $\eta$ which is in some sense "below" $p_{i}$. Also, $\eta$ intersects $\beta_{i}$ "by the left".

Consider the arc $\delta$ from Lemma 4.25 .

Definition 4.30. Let $i \in \mathbf{N}$. We say that an iterate $\widehat{f}^{n}(\tilde{\gamma})$ of $\tilde{\gamma}$ has good intersection with $\widehat{f}^{-N_{1}}\left(\beta_{i}\right)$ if $\widehat{f}^{n}(\tilde{\gamma})$ contains a subarc $\mu$ such that (see Fig. 14):

1. $\mu(0)=\widehat{f}^{n}(\tilde{\gamma}(0))=\tilde{\gamma}(0) \in \operatorname{sing}(\widehat{\mathcal{F}}) \cap \mathcal{C}_{-2}$,

2. $\mu(1) \in T^{i}(\delta) \subset \widehat{f}^{-N_{1}}\left(\beta_{i}\right)$, and

3. $\mu$ is homotopic wfe $\operatorname{Rel}\left(\widehat{f}^{-N_{1}}\left(F_{i}\right)\right)$ to an arc $\nu$ of the form $\nu=\nu_{1} \cdot \nu_{2} \cdot \nu_{3}$, where $\nu_{1}$ is a horizontal arc starting in $\mu(0)$ and ending in the 'strip' $R\left(\mathcal{C}_{i-2}\right) \cap L\left(\mathcal{C}_{i-1}\right), \nu_{2}$ is a vertical arc from $\nu_{1}(1)$ downwards to a point of $T^{i}(\delta)$, and $\nu_{3}$ is the arc contained in $T^{i}(\delta)$ going from $\nu_{2}(1)$ to $\mu(1)$. 


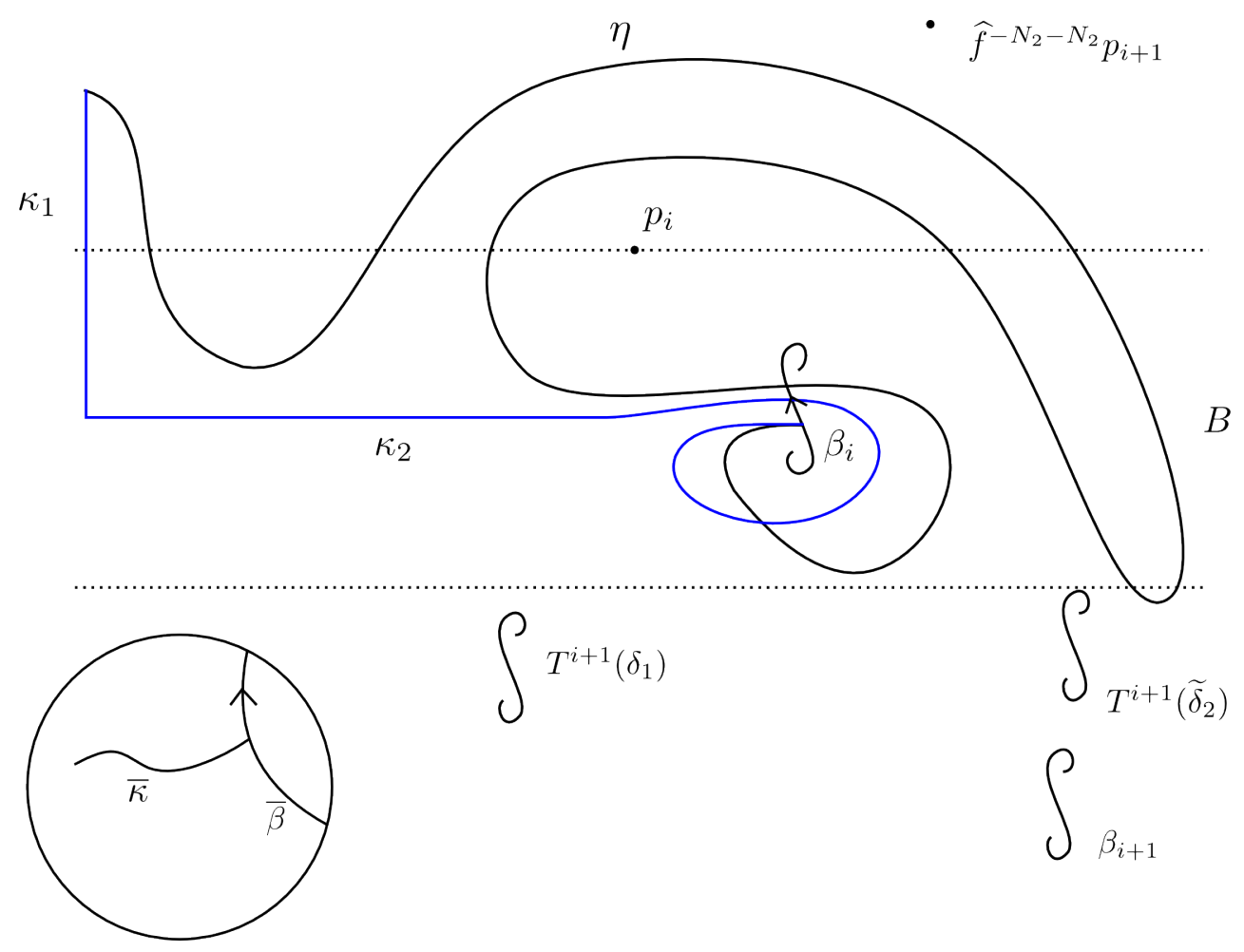

Figure 13: Illustration of an arc which is well positioned with respect to $p_{i}$. Left-bottom corner: the lifts $\bar{\beta}, \bar{\kappa}$ to the universal cover of $\mathbf{R}^{2} \backslash\left(\operatorname{sing}(\widehat{\mathcal{F}}) \cap F_{i}\right)$.

\subsubsection{Induction steps.}

The following two lemmas are the main induction steps in the proof of Theorem D, for the case that $\mathcal{C}$ has singularities.

Lemma 4.31. Let $i \in \mathbf{N}_{0}$. If an iterate $\widehat{f}^{n} \tilde{\gamma}$ of $\tilde{\gamma}$ is well positioned with respect to $p_{i}$, then $\widehat{f}^{n+N_{2}} \tilde{\gamma}$ has good intersection with $\widehat{f}^{-N_{1}} \beta_{i+1}$.

Lemma 4.32. Let $i \in \mathbf{N}$. If an iterate $\widehat{f}^{n} \tilde{\gamma}$ of $\tilde{\gamma}$ has good intersection with $\widehat{f}^{-N_{1}} \beta_{i}$, then $\widehat{f}^{n+N_{1}} \tilde{\gamma}$ is well positioned with respect to $p_{i}$.

\subsubsection{Lemmas 4.31 and 4.32 imply Theorem $D$.}

As we mentioned in 4.6.1 to prove Theorem $\mathrm{D}$ it suffices to prove Lemma 4.24. To prove such lemma from the induction lemmas 4.31 and 4.32 , it suffices to show that $f^{N_{1}}(\widetilde{\gamma})$ is well positioned with respect to $p_{0}$.

Consider the arc $\lambda$ defined in 4.6 .2 and recall that by definition, $\lambda(0) \in \operatorname{sing}(\gamma),\left.\lambda\right|_{[0,1)} \subset$ $L\left(\mathcal{C}_{0}\right)$, and $\lambda(1) \in \beta_{0} \subset \mathcal{C}_{0}$. By definition $\widetilde{\gamma}=\widehat{f}^{-N_{1}} \lambda$, and then $\hat{f}^{N_{1}}(\widetilde{\gamma})=\lambda$. Defining $\eta=\widehat{f}^{N_{1}}(\widetilde{\gamma})=\lambda$ we have that $\eta$ satisfies items 1 and 2 from the definition of being well positioned with respect to $p_{0}$.

We now see that $\eta$ satisfies item 3. By definition, $p_{0}$ is above $\lambda=\eta$. By item 2 from Claim 4.27 we have that $\widehat{f}^{-i\left(N_{1}+N_{2}\right)} p_{i}$ is above $p_{0}$ for all $i>0$, and then $\eta$ is below $\widehat{f}^{-i\left(N_{1}+N_{2}\right)} p_{i}$ for 


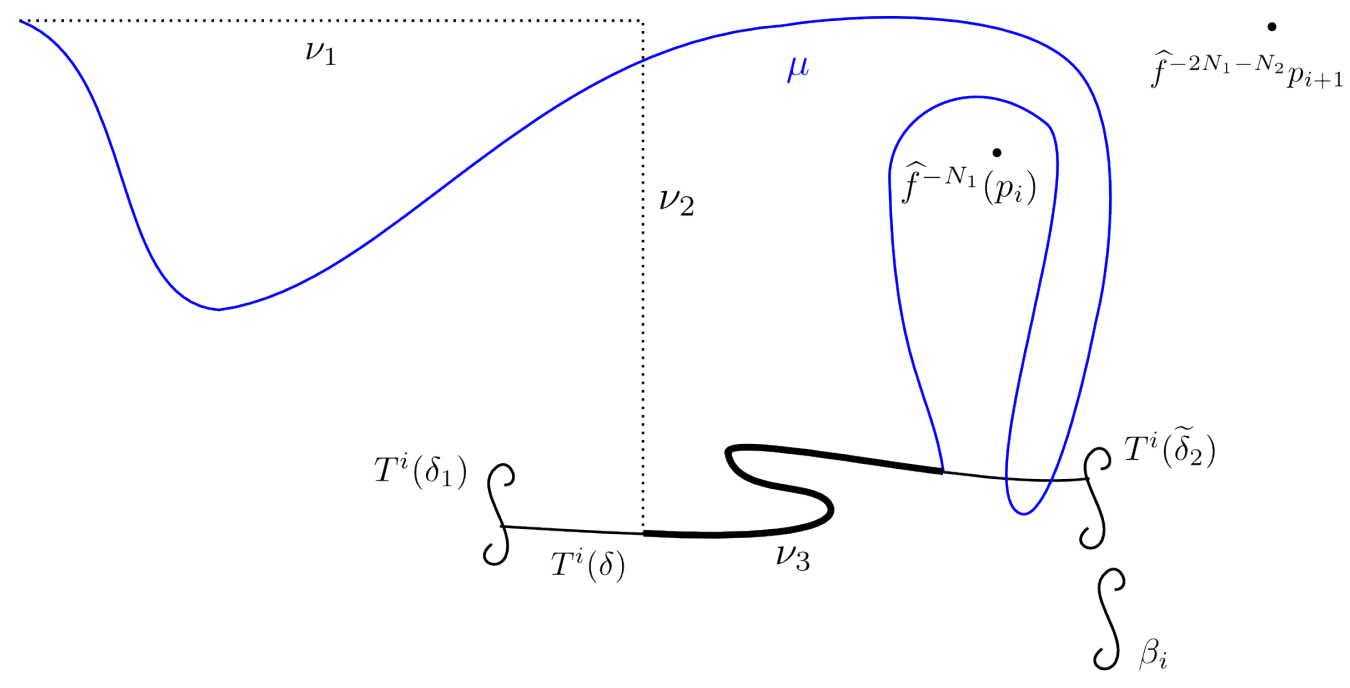

$\int T^{i+1}\left(\delta_{1}\right)$

$T^{i+1}\left(\widetilde{\delta}_{2}\right) \rho$

$\int^{\beta_{i+1}}$

Figure 14: Illustration of an arc which has good intersection with $\widehat{f}^{-N_{1}} \beta_{i}$.

all $i$. Also, from 32 we have that $\lambda=\eta$ is above the sets $\operatorname{sing}\left(\beta_{1}\right), \operatorname{sing}\left(T\left(\delta_{1}\right)\right), \operatorname{sing}\left(T\left(\widetilde{\delta}_{2}\right)\right)$, and then $\eta$ is above $\operatorname{sing}\left(\beta_{i}\right), \operatorname{sing}\left(T^{i}\left(\delta_{1}\right)\right), \operatorname{sing}\left(T^{i}\left(\widetilde{\delta}_{2}\right)\right)$ for all $i \geq 1$. This easily implies that $\eta$ is homotopic wfe $\operatorname{Rel}\left(F_{0}\right)$ to an arc $\kappa=\kappa_{1} \cdot \kappa_{2}$, with $\kappa_{1}$ straight vertical and $\kappa_{2}$ contained in the strip $B_{0}$ from Definition 4.29 . We have then item 3(a) from such definition.

To verify item $3(\mathrm{~b})$, consider a lift $\bar{\kappa}_{2}$ of $\kappa_{2}$ to the universal cover of $\mathbf{R}^{2} \backslash\left(\operatorname{sing}(\widehat{\mathcal{F}}) \cap F_{0}\right)$, and let $\bar{\beta}$ be a lift of $\beta$ containing $\bar{\kappa}_{2}(1)$. We want to see that $\bar{\kappa}_{2}(0) \in L(\bar{\beta})$. Let $\bar{\kappa}_{1}$ be the lift of $\kappa_{1}$ such that $\bar{\kappa}_{1}(1)=\bar{\kappa}_{2}(0)$ and note that as $\kappa(0) \in \operatorname{sing}(\widehat{\mathcal{F}}) \backslash F_{0}$, then $\bar{\kappa}_{1}(0)$ is a singularity of the lift of the foliation $\widehat{\mathcal{F}} \backslash\left(\operatorname{sing}(\widehat{\mathcal{F}}) \cap F_{0}\right)$. Suppose by contradiction that $\bar{\kappa}_{2}(0) \in R(\bar{\beta})$. As $\kappa_{1} \cap \beta=\emptyset$ we then have $\bar{\kappa}_{1}(0)=\bar{\kappa}_{1} \cdot \bar{\kappa}_{2}(0) \in R(\bar{\beta})$. Let $\left(\bar{f}_{t}\right)$ be the canonical lift of the isotopy $\left(\widehat{f}_{t}\right)$. By Lemma 2.17 we have that $\bar{f}^{-N_{1}}\left(\bar{\kappa}_{1} \cdot \bar{\kappa}_{2}\right) \cap \bar{\beta} \neq \emptyset$, and then $\widehat{f}^{-N_{1}}\left(\kappa_{1} \cdot \kappa_{2}\right) \cap \beta \neq \emptyset$. As $\eta$ is homotopic wfe $\operatorname{Rel}\left(F_{0}\right)$ to $\kappa_{1} \cdot \kappa_{2}$, we get that there is a lift $\bar{\eta}$ of $\eta$ such that $\bar{\eta}(0)=\bar{\kappa}_{1} \cdot \bar{\kappa}_{2}(0)$ and $\bar{\eta}(1)=\bar{\kappa}_{1} \cdot \bar{\kappa}_{2}(1)$, and then $\widehat{f}^{-N_{1}} \bar{\eta} \cap \bar{\beta} \neq \emptyset$. Then,

$$
\widehat{f}^{-N_{1}} \lambda \cap \beta=\widehat{f}^{-N_{1}} \eta \cap \beta \neq \emptyset,
$$

which contradicts the definition of $\lambda$. Therefore we must have $\bar{\kappa}_{1} \cdot \bar{\kappa}_{2}(0) \in L(\bar{\beta})$, and then $\bar{\kappa}(0) \in L(\bar{\beta})$ because $\kappa_{1} \cap \beta=\emptyset$, as desired.

\subsubsection{Proof of lemma 4.31}

To simplify the notation, we will prove Lemma 4.31 for the case that $i=0$. Namely, we will prove the following. 
Lemma 4.33. If an iterate $\widehat{f}^{n} \tilde{\gamma}$ of $\tilde{\gamma}$ is well positioned with respect to $p_{0}$, then $\widehat{f}^{n+N_{2}} \tilde{\gamma}$ has good intersection with $\widehat{f}^{-N_{1}} \beta_{1}$.

In the proof of this lemma, the fact that $i=0$ will have nothing special, and therefore the proof of Lemma 4.31 will follow by an identical argument. The rest of this section is devoted to the proof of Lemma 4.33 .

The loops $c_{i}, i \geq 1$. We now fix a family of loops $c_{i}$ that will be used in the following lemma. Recall that the points $p_{i}$ were defined in 34 in a way that the paths $\left\{\widehat{f_{t}}\left(p_{i}\right): t \in \mathbf{R}\right\}$ are pairwise disjoint. Therefore we can choose simple closed curves $c_{i}$, for $i \in \mathbf{N}$, such that (see Fig. 15:

- $\left\{\widehat{f}_{t}\left(\widehat{f}^{-i\left(N_{1}+N_{2}\right)}\left(p_{i}\right)\right): t \in\left[0, N_{2}\right]\right\} \subset \operatorname{int}\left(c_{i}\right)$, where $\operatorname{int}\left(c_{i}\right)$ denotes the bounded connected component of $\mathbf{R}^{2} \backslash c_{i}$,

- $\operatorname{Fill}\left(c_{i}\right) \cap \widehat{f}_{t}\left(F_{0}\right)=\left\{\widehat{f}_{t}\left(\widehat{f}^{-i\left(N_{1}+N_{2}\right)}\left(p_{i}\right)\right)\right\}$ for all $t \in\left[0, N_{2}\right]$,

- $\operatorname{Fill}\left(c_{i}\right) \subset R\left(\mathcal{C}_{i-1}\right) \cap L\left(\mathcal{C}_{i}\right)$.

Also, note that by definition of $p_{0},\left\{\widehat{f}_{t}\left(p_{0}\right): t \in(-\infty, 0]\right\}$ is above $\left\{\widehat{f}_{t} \beta: t \in\left[-N_{1}, 0\right]\right\}$. By periodicity and as $T(\delta) \subset \widehat{f}_{-N_{1}} \beta_{1}$, we may choose $c_{1}$ such that

- Fill $\left(c_{1}\right)$ is above $T(\delta)$.

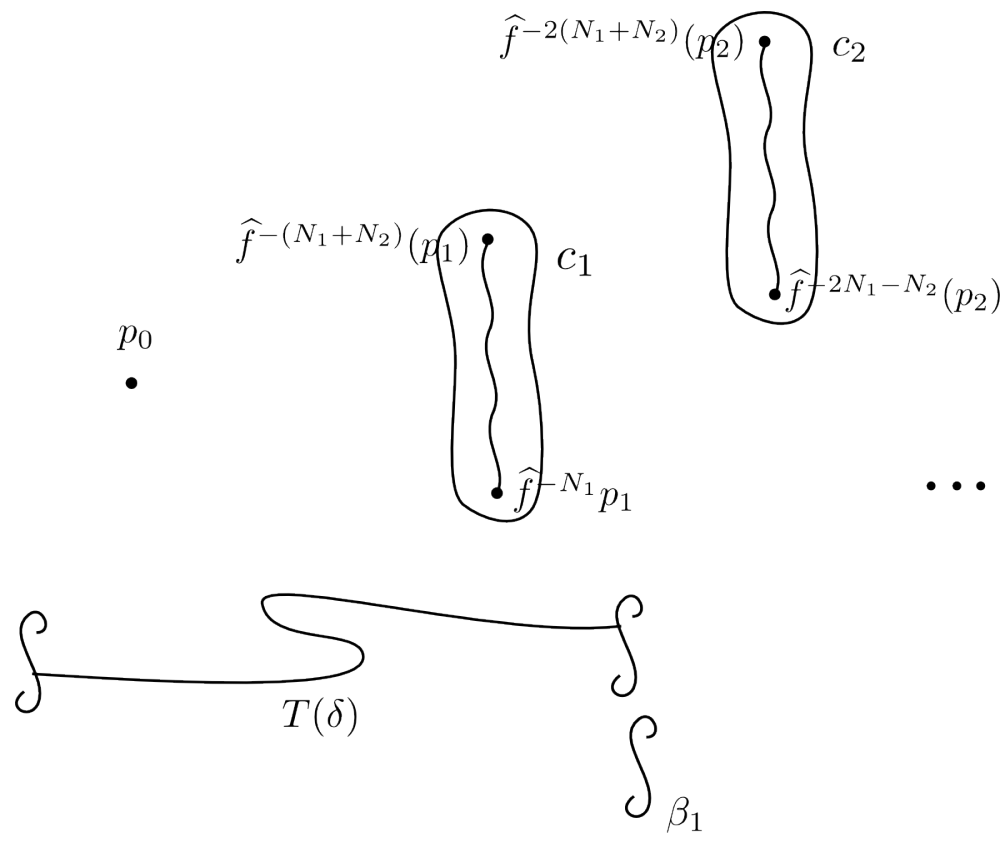

Figure 15: The curves $c_{i}$.

For the next lemma, we also define $E=\left(F_{0} \backslash\left\{p_{0}\right\}\right) \cup\{\tilde{\gamma}(0)\}$, and for $t \in \mathbf{R}$ set

$$
E^{t}=\widehat{f}_{t}(E) \text {. }
$$


Lemma 4.34. There exist a continuous family $\left\{\Pi_{t}\right\}_{t \in\left[0, N_{2}\right]}$ of covering maps $\Pi_{t}: \mathbf{D} \rightarrow \mathbf{R}^{2} \backslash E^{t}$, and an isotopy $\left(\bar{f}_{t}\right)_{t \in\left[0, N_{2}\right]}$ of $\mathbf{D}$, starting in $\bar{f}_{0}=\mathrm{Id}$, such that:

1. For any $0 \leq t \leq N_{2}$, the diagram

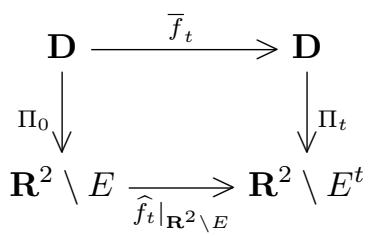

commutes.

2. For all $t \in\left[0, N_{2}\right]$ we have $\left.\Pi_{0}\right|_{\mathbf{D} \backslash C}=\left.\Pi_{t}\right|_{\mathbf{D} \backslash C}$, where $C=\Pi_{0}^{-1}\left(\cup_{k \in \mathbf{N}} \operatorname{int}_{E}\left(c_{k}\right)\right)$ and $\operatorname{int}_{E}\left(c_{k}\right)$ denotes the bounded connected component of $\left(\mathbf{R}^{2} \backslash E\right) \backslash c_{k}$.

3. Let $* \in\left\{\delta_{1}, \widetilde{\delta}_{2}, \beta\right\}$. Then, if $₹$ is a lift of $*$ to $\mathbf{D}$ by $\Pi_{0}$, $₹$ is a Brouwer curve for $\bar{f}_{N_{2}}$.

Proof. Let $P=\left\{\widehat{f}^{-k\left(N_{1}+N_{2}\right)}\left(p_{k}\right): k \in \mathbf{N}\right\}$. Define

$$
E_{1}=E \backslash P=E \cap \operatorname{sing}(\widehat{\mathcal{F}}),
$$

and let $\pi_{1}: \mathbf{D} \rightarrow \mathbf{R}^{2} \backslash E_{1}$ be a covering map. As $E_{1} \subset \operatorname{sing}(\widehat{\mathcal{F}})$, we may consider the canonical $\operatorname{lift}\left(\widetilde{f}_{t}\right)$ by $\pi_{1}$ of the isotopy $\left.\widehat{f}_{t}\right|_{\mathbf{R}^{2} \backslash E_{1}}$. Also, the Brouwer foliation $\left.\widehat{\mathcal{F}}\right|_{\mathbf{R}^{2} \backslash E_{1}}$ (with singularities) lifts by $\pi_{1}$ to a foliation of $\mathbf{D}$ (also with singularities) which is transverse to the isotopy $\widetilde{f}_{t}$. For every $t$, the following diagram commutes:

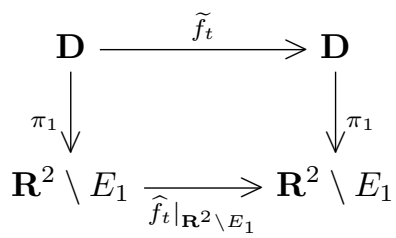

For $t \in\left[0, N_{2}\right]$, let $P^{t}=\widehat{f}_{t}(P)$ and define

$$
D^{t}=\mathbf{D} \backslash \pi_{1}^{-1}\left(P^{t}\right)
$$

As $E=E_{1} \cup P$, considering the restrictions $\left.\widehat{f}_{t}\right|_{\mathbf{R}^{2} \backslash E},\left.\pi_{1}\right|_{D^{0}}$ and $\left.\tilde{f}_{t}\right|_{D^{0}}$, we obtain the following commutative diagram

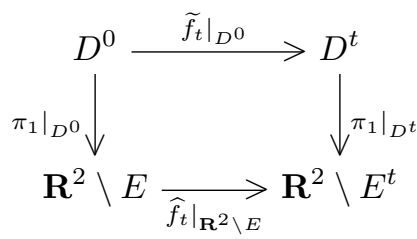

for $t \in\left[0, N_{2}\right]$.

Using standard techniques from plane topology, one may construct an isotopy $\left(J_{t}\right)_{t \in\left[0, N_{2}\right]}$ on $\mathbf{R}^{2}$ starting in the identity and such that

- $J_{t}(x)=x$ for all $t \in\left[0, N_{2}\right]$ and all $x \in \mathbf{R}^{2} \backslash \cup_{k \in \mathbf{N}} \operatorname{int}\left(c_{k}\right)$, and 
- $J_{t}\left(p_{k}\right)=\widehat{f}_{t}\left(p_{k}\right)$ for all $t \in\left[0, N_{2}\right]$ and all $k \in \mathbf{N}$.

Consider the restriction $\left(\left.J_{t}\right|_{\mathbf{R}^{2} \backslash E_{1}}\right)_{t \in\left[0, N_{2}\right]}$, which is an isotopy on $\mathbf{R}^{2} \backslash E_{1}$, and consider the canonical lift $\left(\widetilde{J}_{t}\right)_{t \in\left[0, N_{2}\right]}$ by $\pi_{1}$ of $\left.J_{t}\right|_{\mathbf{R}^{2} \backslash E_{1}}$. Defining

$$
F_{t}=\widetilde{J}_{t}^{-1} \circ \widetilde{f}_{t}
$$

we obtain an isotopy $\left(F_{t}\right)_{t \in\left[0, N_{2}\right]}$ on $\mathbf{D}$. By the properties of $J_{t}, F_{t}\left(D^{0}\right)=D^{0}$ for all $t$, and then we have an isotopy $\left(\left.F_{t}\right|_{D^{0}}\right)_{t \in\left[0, N_{2}\right]}$ on $D^{0}$.

Consider a covering map $\pi_{2}: \mathbf{D} \rightarrow D^{0}$, and the canonical lift $\left(\bar{f}_{t}\right)$ by $\pi_{2}$ of the isotopy $\left(\left.F_{t}\right|_{D^{0}}\right)$, so

$$
\pi_{2} \circ \bar{f}_{t}=\left.F_{t}\right|_{D^{0}} \circ \pi_{2} .
$$

For $t \in\left[0, N_{2}\right]$, define the covering map $\pi_{2}^{t}: \mathbf{D} \rightarrow D^{t}$ by

$$
\pi_{2}^{t}=\left.\widetilde{J}_{t}\right|_{D^{0}} \circ \pi_{2}
$$

As $J_{0}=$ Id we have that $\pi_{2}^{0}=\pi_{2}$. The maps $\pi_{2}^{t}$ vary continuously, and we have

$$
\begin{array}{rlr}
\pi_{2}^{t} \circ \bar{f}_{t} & =\left.\widetilde{J}_{t}\right|_{D^{0}} \circ \pi_{2} \circ \bar{f}_{t}=\left.\left.\widetilde{J}_{t}\right|_{D^{0}} \circ F_{t}\right|_{D^{0}} \circ \pi_{2} \quad \text { by }(37) \\
& =\left.\left.\left.\widetilde{J}_{t}\right|_{D^{0}} \circ \widetilde{J}_{t}^{-1}\right|_{D^{t}} \circ \widetilde{f}_{t}\right|_{D^{0}} \circ \pi_{2} \\
& =\left.\operatorname{Id}_{D^{t}} \circ \widetilde{f}_{t}\right|_{D^{0}} \circ \pi_{2}=\left.\widetilde{f}_{t}\right|_{D^{0}} \circ \pi_{2} .
\end{array}
$$

Thus, for every $t \in\left[0, N_{2}\right]$, the following diagram commutes

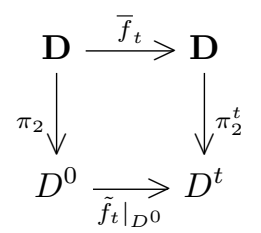

If we define $\Pi_{t}: \mathbf{D} \rightarrow \mathbf{R}^{2} \backslash E^{t}$ for $t \in\left[0, N_{2}\right]$ by

$$
\Pi_{t}=\left.\pi_{1}\right|_{D^{t}} \circ \pi_{2}^{t}
$$

we have that the maps $\Pi_{t}$ vary continuously, and $\Pi_{0}=\left.\pi_{1}\right|_{D^{0}} \circ \pi_{2}^{0}=\left.\pi_{1}\right|_{D^{0}} \circ \pi_{2}$. Therefore combining the last two commutative diagrams we obtain that

$$
\Pi_{t} \circ \bar{f}_{t}=\left.\widehat{f}_{t}\right|_{\mathbf{R}^{2} \backslash E} \circ \Pi_{0} \quad \text { for } t \in\left[0, N_{2}\right],
$$

which proves Item 1 of the lemma ${ }^{4}$

To prove Item 2, first note that, as

$$
J_{t}(x)=x \quad \forall t \in\left[0, N_{2}\right] \quad \forall x \in \mathbf{R}^{2} \backslash \cup_{k \in \mathbf{N}} \operatorname{int}\left(c_{k}\right),
$$

we have that

$$
\widetilde{J}_{t}(x)=x \quad \forall t \in\left[0, N_{2}\right] \quad \forall x \in \mathbf{D} \backslash C_{1},
$$

where $C_{1}=\left(\pi_{1}\right)^{-1}\left(\cup_{k \in \mathbf{N}} \operatorname{int}\left(c_{k}\right)\right)$. By this, if

$$
C=\pi_{2}^{-1}\left(C_{1} \cap D^{0}\right)=\Pi_{0}^{-1}\left(\cup_{k \in \mathbf{N}} \operatorname{int}_{E}\left(c_{k}\right)\right),
$$

\footnotetext{
${ }^{4}$ So far the intermediate covering $\pi_{1}$ might seem unnecesary. However, it will be used in the proof of item 3.
} 
we have that

$$
\pi_{2}^{t}(x)=\left.\widetilde{J}_{t}\right|_{D^{0}} \circ \pi_{2}(x)=\pi_{2}(x) \quad \forall t \in\left[0, N_{2}\right] \quad \forall x \in \mathbf{D} \backslash C .
$$

Observe also that $\mathbf{D} \backslash C_{1} \subset D^{t}$ for all $t \in\left[0, N_{2}\right]$, and in particular

$$
\left.\pi_{1}\right|_{D^{t}} \circ \pi_{2}(x)=\left.\pi_{1}\right|_{D^{0}} \circ \pi_{2}(x) \quad \forall t \in\left[0, N_{2}\right] \quad \forall x \in \mathbf{D} \backslash C .
$$

Thus, for all $t \in\left[0, N_{2}\right]$ and all $x \in \mathbf{D} \backslash C$ we have

$$
\begin{aligned}
\Pi_{t}(x)=\left.\pi_{1}\right|_{D^{t}} \circ \pi_{2}^{t}(x) & =\left.\pi_{1}\right|_{D^{t}} \circ \pi_{2}(x) & & \text { by } 38 \\
& =\left.\pi_{1}\right|_{D^{0}} \circ \pi_{2}(x) & & \text { by } 39 \\
& =\Pi_{0}(x) . & &
\end{aligned}
$$

This proves Item (2).

To prove Item 3 , we will now see that, if $\bar{\beta}$ is a lift by $\Pi_{0}$ of $\beta$, then $\bar{\beta}$ is a Brouwer line for $\bar{f}_{N_{2}}$. The same argument will hold for $\delta_{1}$ and $\widetilde{\delta}_{2}$.

As we saw above, the isotopy $\left(\left.\widehat{f}_{t}\right|_{\mathbf{R}^{2} \backslash E_{1}}\right)$ lifts by $\pi_{1}$ to its canonical lift $\left(\widetilde{f}_{t}\right)$ on $\mathbf{D}$, and the Brouwer foliation $\widehat{\mathcal{F}}$ lifts to a foliation of $\mathbf{D}$ with singularities which is transverse to $\widetilde{f}_{t}$. In particular, if $\beta^{\prime}$ is a lift by $\pi_{1}$ of $\beta$, then $\beta^{\prime}$ is a Brouwer line for $\widetilde{f}_{N_{2}}$. Observe also that, as $\beta$ is disjoint from $P$, then $\pi_{1}^{-1}(\beta)=\left(\left.\pi_{1}\right|_{D^{0}}\right)^{-1}(\beta)$. $\emptyset$.

Now, as $\beta^{\prime}$ is a Brouwer line for $\widetilde{f}_{N_{2}}$, we have that if $\beta^{\prime \prime}$ is a lift of $\beta^{\prime}$ by $\pi_{2}^{0}$, then $\bar{f}_{N_{2}}\left(\beta^{\prime \prime}\right) \cap \beta^{\prime \prime}=$

Thus, any lift $\beta^{\prime \prime}$ of $\beta$ by $\Pi_{0}=\left.\pi_{1}\right|_{D^{0}} \circ \pi_{2}^{0}$ is disjoint from its image by $\bar{f}_{N_{2}}$, and to prove Item 3 it suffices to show that $\bar{f}_{N_{2}}\left(\beta^{\prime \prime}\right)$ cannot be contained in $L\left(\beta^{\prime \prime}\right)$. However, this is a simple consequence of the fact that the maps $\pi_{1} \mid D^{0}$ and $\pi_{2}^{t}$ preserve orientation, and that $\beta^{\prime}$ is a Brouwer line for $\widetilde{f}_{N_{2}}$. This proves item 3 , and finishes the proof of the lemma.

The $\operatorname{arc} S$ and the loop $c_{0}$. Recall our notation from Lemma 4.34 for $E=\left(F_{0} \backslash\left\{p_{0}\right\}\right) \cup\{\widetilde{\gamma}(0)\}$. By construction, the path $\left(\widehat{f}_{t}\left(p_{0}\right)\right)_{t \in\left[0, N_{2}\right]}$ is contained in the straight vertical strip $\left(\ell_{1}, \ell_{2}\right) \subset$ $R\left(\mathcal{C}_{-1}\right) \cap L\left(\mathcal{C}_{0}\right)$ (see (31)). As $E$ is disjoint from $R\left(\mathcal{C}_{-1}\right) \cap L\left(\mathcal{C}_{0}\right)$, we have that

$$
\left(\widehat{f}_{t}\left(p_{0}\right)\right)_{t \in\left[0, N_{2}\right]} \text { is homotopic wfe } \operatorname{Rel}(E) \text { to a straight vertical arc } S \text {. }
$$

Let $c_{0}$ be a closed loop oriented counterclock-wise containing $\widetilde{\gamma}(0)$ in its interior, and such that $c_{0} \subset L\left(\mathcal{C}_{-1}\right)$. Then, by definition of the sets $F_{i}$, Fill $\left(c_{0}\right) \cap F_{i}=\emptyset$ for all $i \geq 0$.

Lifting to $\mathbf{D}$ by $\Pi_{0}$. First recall that as we are assuming that $\widehat{f}^{n}(\widetilde{\gamma})$ is well positioned with respect to $p_{0}$, there exists a subarc $\eta \subset \widehat{f}^{n}(\widetilde{\gamma})$ which is homotopic wfe to an arc $\kappa=\kappa_{1} \cdot \kappa_{2}$ satisfying the properties from definition 4.29 .

Fix a lift $\bar{\kappa}$ of $\left.\kappa\right|_{(0,1]}$ to $\mathbf{D}$ by $\Pi_{0}: \mathbf{D} \rightarrow \mathbf{R}^{2} \backslash E$. Let $\bar{\beta}$ be a lift of $\beta$ to $\mathbf{D}$ by $\Pi_{0}$ that contains the point $\bar{\kappa}(1)$ (see Fig. 16). Let $q_{3}=\min \left\{t \in(0,1]: \kappa(t) \in c_{0}\right\}$, and define $\bar{c}_{0}$ as a lift of $c_{0}$ such that $\bar{c}_{0}$ contains $\bar{\kappa}\left(q_{3}\right)$. Note that, as $c_{0}$ is oriented counterclock-wise,

$$
\bar{\kappa}(t) \in L\left(\bar{c}_{0}\right) \quad \text { for } t \in\left(0, q_{3}\right) .
$$

Define then $\bar{\eta}$ as the lift of $\left.\eta\right|_{(0,1]}$ by $\Pi_{0}$ such that $\bar{\eta}(1)=\bar{\kappa}(1)$, and note that, as $\eta$ and $\kappa$ are homotopic wfe $\operatorname{Rel}\left(F_{0}\right)$, and by 40 ,

$$
\bar{\eta}(t) \in L\left(\bar{c}_{0}\right), \quad \text { for } t \text { small enough. }
$$

Observe that as $\widehat{f}^{N_{2}} p_{0}$ is below $T(\delta)$ (see Claim 4.27) and by definition of $\kappa$ and $S$, the arc $S$ intersects $\kappa$ and $T(\delta)$. 
Let

$$
q_{4}=\min \{t \in(0,1]: \kappa(t) \in S\},
$$

and let $q_{5}$ be such that $S\left(q_{5}\right)=\kappa\left(q_{4}\right)$. Let $\bar{S}$ be the lift of $S$ by $\Pi_{0}$ such that $\bar{S}\left(q_{5}\right) \in \bar{\kappa}\left(q_{4}\right)$. Note that, as $S$ is homotopic wfe $\operatorname{Rel}(E)$ to $\left(\widehat{f}_{t}\left(p_{0}\right)\right)_{t \in\left[0, N_{2}\right]}$, we have that

$$
\bar{S}(1)=\bar{f}_{N_{2}}(\bar{S}(0)) .
$$

Let $q_{6}, t_{*} \in[0,1]$ be such that $S\left(q_{6}\right)=T \delta\left(t_{*}\right)$, and let $\bar{\delta}$ be the lift of $T(\delta)$ by $\Pi_{0}$ such that $\bar{S}\left(q_{6}\right)=\bar{\delta}\left(t_{*}\right)$. Also, let $\bar{\delta}_{1}$ be a lift of $\delta_{1}$ by $\Pi_{0}$ such that

$$
\bar{\delta}(0) \in \bar{\delta}_{1} .
$$

In case $\widetilde{\delta}_{2} \neq \emptyset$, let $\bar{\delta}_{2}$ be a lift of $\widetilde{\delta}_{2}$ by $\Pi_{0}$ such that

$$
\bar{\delta}(1) \in \bar{\delta}_{2},
$$

and in case $\widetilde{\delta}_{2}=\emptyset$ define $\bar{\delta}_{2}=\emptyset$.

Finally, let $\bar{f}_{t}: \mathbf{D} \rightarrow \mathbf{D}$ and $\Pi_{t}: \mathbf{D} \rightarrow \mathbf{R}^{2} \backslash E^{t}$ be as in Lemma 4.34

Main claims. The following two claims will finish the proof of Lemma 4.33

Claim 4.35. If $\bar{f}_{N_{2}}(\bar{\eta}) \cap \bar{\delta} \neq \emptyset$, then $\widehat{f}^{N_{2}}(\eta)$ has good intersection with $\widehat{f}^{-N_{1}}\left(\beta_{1}\right)$.

Claim 4.36. $\bar{f}_{N_{2}}(\bar{\eta}) \cap \bar{\delta} \neq \emptyset$.

To prove these claims, we begin with the following lemma.

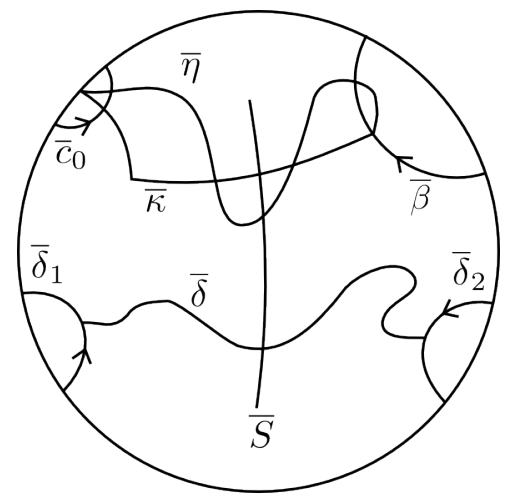

Figure 16: Lifting by $\Pi_{0}$.

Lemma 4.37. The following hold:

1. For all $t \in\left[0, N_{2}\right]$ we have $\left.\Pi_{t}\right|_{A}=\left.\Pi_{0}\right|_{A}$, where $A=\bar{L}\left(\bar{c}_{0}\right) \cup \bar{\kappa} \cup \bar{S} \cup \bar{\delta} \cup \bar{\delta}_{1} \cup \bar{\delta}_{2}$.

2. Let $* \in\left\{\delta, \delta_{1}, \widetilde{\delta}_{2}\right\}$. If $\bar{f}_{N_{2}}(\bar{\eta}) \cap \bar{*} \neq \emptyset$ then $\widehat{f}^{N_{2}}(\eta) \cap * \neq \emptyset$.

3. $\forall n \in \mathbf{Z} \quad \exists a>0$ such that $\left(\bar{f}_{1}\right)^{n} \bar{\eta}(t) \in L\left(\bar{c}_{0}\right)$, for $0<t<a$.

4. Each of the arcs $\bar{S}, \bar{\delta}, \bar{\delta}_{1}$ and $\bar{\delta}_{2}$ is contained in $R\left(\bar{c}_{0}\right)$. 
Proof. To prove item 1 just note that $\Pi_{0}(A) \cap \operatorname{int}\left(c_{k}\right)=\emptyset$ for all $k>0$, by definition of the curves $c_{k}$, and therefore, by Item 2 from Lemma 4.34 we have that $\left.\Pi_{t}\right|_{A}=\left.\Pi_{0}\right|_{A}$, for all $t \in\left[0, N_{2}\right]$.

By this, item 1 of Lemma 4.34 implies that

$$
\left.\Pi_{t} \circ \bar{f}_{t}\right|_{A}=\left.\widehat{f}_{t} \circ \Pi_{0}\right|_{A} \quad \forall t \in\left[0, N_{2}\right]
$$

which implies item 2. Also by Lemma 4.34 .

$$
\Pi_{0} \circ \bar{f}_{-t}=\left(\left.\widehat{f}_{t}\right|_{\mathbf{R}^{2} \backslash E}\right)^{-1} \circ \Pi_{t} \quad \forall t \in\left[0, N_{2}\right] .
$$

Item 3 is an easy consequence of $\sqrt{45}, \sqrt{46}$ and the fact that for all $t \in \mathbf{R}$ the arc $\widehat{f}_{t} \eta$ is issued from $\eta(0)=\widetilde{\gamma}(0) \in \operatorname{int}\left(c_{0}\right)$, as $c_{0}$ is oriented counterclock-wise.

To prove Item 4 , note that by definition of $c_{0}$, the set Fill $\left(c_{0}\right)$ does not intersect any of the sets $T(\delta), \delta_{1}, \widetilde{\delta}_{2}$ and $S$, and then the lifts $\bar{S}, \bar{\delta}, \bar{\delta}_{1}$ and $\bar{\delta}_{2}$ are contained in $R\left(\bar{c}_{0}\right)$.

Proof of Claim 4.35. Without loss of generality, we may make the following assumption on the covering map $\Pi_{0}: \mathbf{D} \rightarrow \mathbf{R}^{2} \backslash E$. If a sequence of loops $\sigma_{i} \subset \operatorname{int}\left(c_{0}\right)$ is such that $\eta(0) \in \operatorname{int}\left(\sigma_{i}\right)$ for all $i$ and $\operatorname{diam}\left(\sigma_{i}\right) \rightarrow 0$ as $i \rightarrow \infty$, and if $\bar{\sigma}_{i}$ denotes the lift of $\sigma_{i}$ contained in $L\left(\bar{c}_{0}\right)$, then $\operatorname{diam}\left(\bar{\sigma}_{i}\right) \rightarrow 0$, as $i \rightarrow \infty$, where in both cases $\operatorname{diam}(X)$ denotes the euclidean diameter of a set $X$.

By this assumption, the limit $\lim _{t \rightarrow 0} \bar{\eta}(t)=: z_{0}$ exists, $z_{0} \in \partial \mathbf{D} \subset \mathbf{C}$, and we may extend the curve $\bar{\eta}:(0,1] \rightarrow \mathbf{D}$ continuously to a curve $\bar{\eta}:[0,1] \rightarrow \mathbf{D} \cup\left\{z_{0}\right\}$ as $\bar{\eta}(0)=z_{0}$. Analogously, for any $t$ extend $\bar{f}_{t} \bar{\eta}$ continuously to a curve $\bar{f}_{t} \bar{\eta}:[0,1] \rightarrow \mathbf{D} \cup\left\{z_{0}\right\}$.

Define

$$
q_{8}=\min \left\{t: \bar{f}_{N_{2}} \bar{\eta}(t) \in \bar{\delta}\right\}
$$

(such minimum exists, as by item 4 from Lemma $4.37 \bar{\delta} \subset R\left(\bar{c}_{0}\right)$ ). As $\mathbf{D} \cup\left\{z_{0}\right\}$ is simply connected, the arc $\left.\bar{f}_{N_{2}} \bar{\eta}\right|_{\left[0, q_{8}\right]}$ is homotopic wfe to the $\operatorname{arc} \bar{\epsilon}=\bar{\epsilon}_{1} \cdot \bar{\epsilon}_{2} \cdot \bar{\epsilon}_{3}$, where the arcs $\bar{\epsilon}_{i}$ are such that:

- $\bar{\epsilon}_{1} \subset \bar{\kappa}$ going from $\bar{\epsilon}_{1}(0)=\bar{f}_{N_{2}} \bar{\eta}(0)=\bar{\eta}(0)$ to $\bar{\epsilon}_{1}(1)=\bar{\kappa}\left(q_{4}\right)$, with $q_{4}$ as in 42 ;

- $\bar{\epsilon}_{2} \subset \bar{S}$ going from $\bar{\epsilon}_{1}(1)$ to $\bar{\epsilon}_{2}(1)=\bar{S}\left(q_{9}\right)$, where $q_{9}=\min \left\{t \in[0,1]: \bar{S}\left(q_{9}\right) \in \bar{\delta}\right\}$;

- $\bar{\epsilon}_{3} \subset \bar{\delta}$ going from $\bar{\epsilon}_{2}(1)$ to $\bar{\epsilon}_{3}(1)=\bar{f}_{N_{2}} \bar{\eta}\left(q_{8}\right)$.

By item 3 from Lemma 4.37 we may define

$$
q_{7}=\min \left\{t: \bar{f}_{N_{2}} \bar{\eta}(t) \in \bar{c}_{0}\right\} .
$$

Define the arc $\epsilon_{1} \subset \mathbf{R}^{2}$ by $\epsilon_{1}(0)=\eta(0)$ and $\epsilon_{1}(t)=\Pi_{N_{2}}\left(\bar{\epsilon}_{1}(t)\right)$ for $t \in(0,1]$. For $i=2,3$, let $\epsilon_{i}=\Pi_{N_{2}}\left(\bar{\epsilon}_{i}\right)$, and define $\epsilon=\epsilon_{1} \cdot \epsilon_{2} \cdot \epsilon_{3}$.

The homotopy wfe from $\left.\bar{f}_{N_{2}} \bar{\eta}\right|_{\left[0, q_{8}\right]}$ to $\bar{\epsilon}$ and the projection $\Pi_{N_{2}}$ induce a homotopy wfe $\operatorname{Rel}\left(E^{N_{2}}\right)$ from $\left.\widehat{f}^{N_{2}} \eta\right|_{\left[0, q_{8}\right]}$ to $\epsilon$ (recall that $E^{t}=\widehat{f}_{t}(E)$ ). By Item 1 from Lemma 4.37

$$
\left.\Pi_{0}\right|_{\bar{\kappa} \cup \bar{S} \cup \bar{\delta}}=\left.\Pi_{t}\right|_{\bar{\kappa} \cup \bar{S} \cup \bar{\delta}} \quad \forall t \in\left[0, N_{2}\right],
$$

and then $\epsilon_{1} \subset \kappa, \epsilon_{2} \subset S$, and $\epsilon_{3} \subset T(\delta)$. Also, by definition of $\epsilon_{1}$ and $E^{N_{2}}$, the arc $\epsilon_{1}$ is homotopic wfe $\operatorname{Rel}\left(E^{N_{2}}\right)$ to the $\operatorname{arc} \tau=\tau_{1} \cdot \tau_{2}$, where $\tau_{1}$ is straight horizontal and $\tau_{2}$ is straight vertical (see Fig. 17). Then,

$\epsilon$ is homotopic wfe $\operatorname{Rel}\left(E^{N_{2}}\right)$ to $\tau_{1} \cdot \tau_{2} \cdot \epsilon_{2} \cdot \epsilon_{3}$. 
As $\left.\widehat{f}^{N_{2}} \eta\right|_{\left[0, q_{8}\right]}$ is homotopic wfe $\operatorname{Rel}\left(E^{N_{2}}\right)$ to $\epsilon$, we obtain that

$$
\left.\widehat{f}^{N_{2}} \eta\right|_{\left[0, q_{8}\right]} \text { is homotopic wfe } \operatorname{Rel}\left(E^{N_{2}}\right) \text { to } \tau_{1} \cdot \tau_{2} \cdot \epsilon_{2} \cdot \epsilon_{3} .
$$

Note that, as $E^{N_{2}} \supset \widehat{f}^{-N_{1}}\left(F_{1}\right)$, the homotopy in 47 holds in particular $\operatorname{Rel}\left(\widehat{f}^{-N_{1}}\left(F_{1}\right)\right)$. As

- $\tau_{1}$ is straight horizontal,

- $\tau_{2} \cdot \epsilon_{2}$ is straight vertical, and

- $\epsilon_{3} \subset T(\delta)$,

we have that $\widehat{f}^{N_{2}+n} \widetilde{\gamma} \supset \widehat{f}^{N_{2}} \eta$ satisfies the definition of good intersection with $\widehat{f}^{-N_{1}}\left(\beta_{1}\right)$, with $\mu=\left.\widehat{f}^{N_{2}} \eta\right|_{\left[0, q_{8}\right]}, \nu_{1}=\tau_{1}, \nu_{2}=\tau_{2} \cdot \epsilon_{2}$ and $\nu_{3}=\epsilon_{3}$. This proves Claim 4.35 .

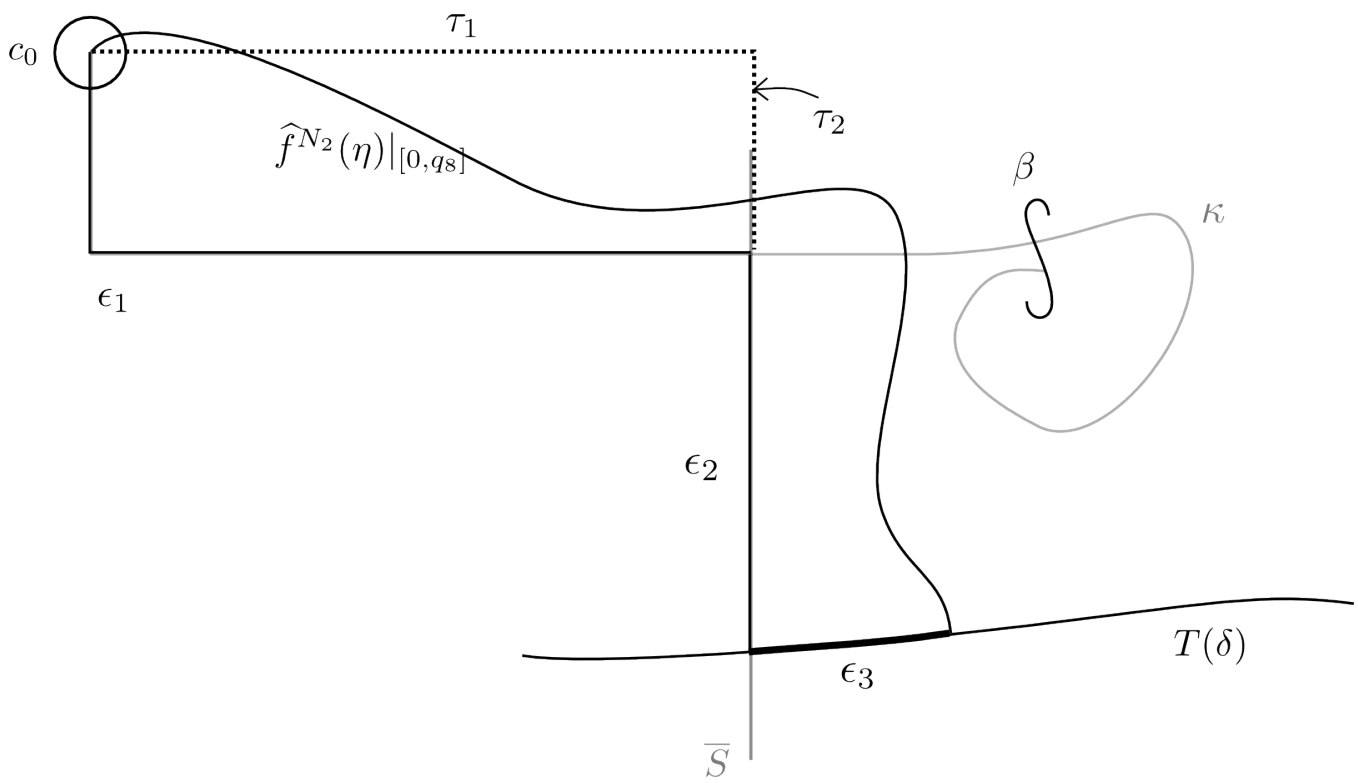

Figure 17: The arcs $\epsilon_{i}$ and $\tau_{i}$ from the proof of Claim 4.35.

Our objective now is to prove Claim 4.36. To this end, we first make some definitions and prove a lemma related to them. Let

$$
\bar{\beta}^{\prime}=\left.\bar{\beta}\right|_{\left[q_{10}, 1\right)},
$$

where $q_{10}$ is such that $\bar{\beta}\left(q_{10}\right)=\bar{\eta}(1)$ (see Fig. 18). Recall our definitions of $\bar{\delta}_{1}, \bar{\delta}_{2}$ from 43, (44). Let

$$
\bar{\delta}_{6}=\left.\bar{\delta}_{1}\right|_{\left[q_{11}, 1\right)},
$$

where $q_{11}$ is such that $\bar{\delta}_{1}\left(q_{11}\right)=\bar{\delta}(0)$. If $\widetilde{\delta}_{2} \neq \emptyset$, define

$$
\bar{\delta}_{7}=\left.\bar{\delta}_{2}\right|_{\left(0, q_{12}\right]},
$$

where $q_{12}$ satisfies $\bar{\delta}_{2}\left(q_{12}\right)=\bar{\delta}(1)$, and in case $\widetilde{\delta}_{2}=\emptyset$ define $\bar{\delta}_{7}=\emptyset$. 


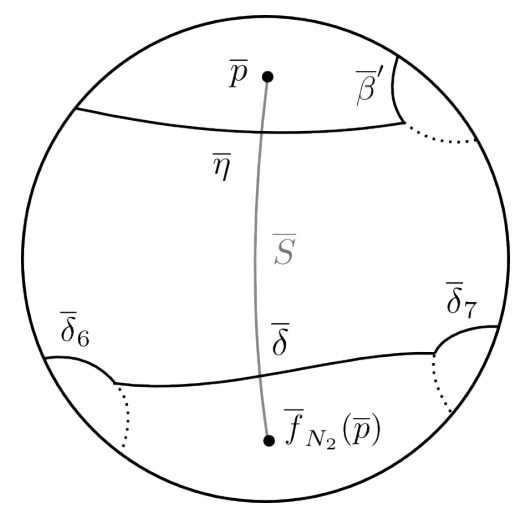

Figure 18: The $\operatorname{arcs} \bar{\beta}^{\prime}, \bar{\delta}_{6}$ and $\bar{\delta}_{7}$ (in the case $\bar{\delta}_{7} \neq \emptyset$ ). From Lemma $4.38, \bar{p} \in L\left(\bar{\eta} \cdot \bar{\beta}^{\prime}\right.$ ) and $\bar{f}_{N_{2}}(\bar{p}) \in R(\Delta)$.

Define $\bar{p} \in \mathbf{D}$ as the lift of $p_{0}$ by $\Pi_{0}$ given by

$$
\bar{p}=\bar{S}(0) .
$$

Let $\theta \subset \mathbf{R}^{2}$ be the straight vertical line passing through $p_{0}$, oriented downwards. Then, $c_{0} \subset R(\theta)$ and $\beta \subset L(\theta)$. Let $\bar{\theta} \subset \mathbf{D}$ be a lift of $\theta$ by $\Pi_{0}$ passing through $\bar{p}$. It is easy to see that

$$
\bar{c}_{0} \subset R(\bar{\theta}) \text { and } \bar{\beta} \subset L(\bar{\theta}) \text {. }
$$

Define

$$
\Delta=\bar{\delta}_{6}^{-1} \cdot \bar{\delta} \cdot \bar{\delta}_{7}^{-1}
$$

Recall that IN $\left(\Gamma_{1}, \Gamma_{2}\right)$ denotes the intersection number of two curves $\Gamma_{1}$ and $\Gamma_{2}$ with disjoint ends (cf. Section 2.8). Without loss of generality we may assume that the curves $\Delta, \bar{\eta} \cdot \bar{\beta}^{\prime}, \bar{\theta}$ have disjoint ends, so their intersection number is well defined.

Lemma 4.38. The following hold:

1. $\operatorname{IN}\left(\bar{\theta}, \bar{f}_{N_{2}}\left(\bar{\eta} \cdot \bar{\beta}^{\prime}\right)\right)=1$,

2. $\bar{p} \in L\left(\bar{\eta} \cdot \bar{\beta}^{\prime}\right)$,

3. $\bar{f}_{N_{2}}(\bar{p}) \in R(\Delta)$, and

4. $\bar{L}\left(\bar{c}_{0}\right) \cup \bar{R}(\bar{\beta}) \subset L(\Delta)$.

Proof. By item 3 from Lemma 4.37, $\bar{f}_{N_{2}} \bar{\eta}(t) \in L\left(\bar{c}_{0}\right)$ for $t$ small. By item 3 from Lemma $4.34, \bar{\beta}$ is a Brouwer line for $\bar{f}_{N_{2}}$, and then $\bar{f}_{N_{2}}\left(\bar{\eta} \cdot \bar{\beta}^{\prime}\right) \subset R(\bar{\beta})$. By this and by $(48), \operatorname{IN}\left(\bar{\theta}, \bar{f}_{N_{2}}\left(\bar{\eta} \cdot \bar{\beta}^{\prime}\right)=1\right.$, which proves Item 1

We now prove Item 2 Recall that, by definition, $\eta$ is homotopic wfe $\operatorname{Rel}\left(F_{0}\right)$ to an arc $\kappa=\kappa_{1} \cdot \kappa_{2}$, where $\kappa_{1} \subset L\left(\mathcal{C}_{-1}\right)$ is straight vertical and $\kappa_{2}$ is below $p_{0}$. Analogously as in Item 1. it follows

$$
\operatorname{IN}\left(\bar{\theta}, \bar{\kappa} \cdot \bar{\beta}^{\prime}\right)=1 .
$$


By definition, $p_{0}$ is above $\kappa_{2} \cup \beta$, and then if $q_{13} \in \mathbf{R}$ is such that $\bar{\theta}\left(q_{13}\right)=\bar{p}$, we have that

$$
q_{13}<\min \left\{t \in \mathbf{R}: \bar{\theta}(t) \in \bar{\kappa} \cdot \bar{\beta}^{\prime}\right\} .
$$

By 449, 50 and by Lemma 2.23 we have

$$
\bar{p} \in L\left(\bar{\kappa} \cdot \bar{\beta}^{\prime}\right) .
$$

As $\kappa$ is homotopic wfe $\operatorname{Rel}\left(F_{0}\right)$ to $\eta$, and as $p_{0} \in F_{0}$, we conclude that

$$
\bar{p} \in L\left(\bar{\eta} \cdot \bar{\beta}^{\prime}\right),
$$

which proves item 2

We now prove Item 3. Analogously as in Item 1, we have

$$
\operatorname{IN}(\bar{\theta}, \Delta)=1 .
$$

Also, as $\widehat{f}^{N_{2}}\left(p_{0}\right)$ is below $T(\delta)$, we have that

$$
\max \{t \in \mathbf{R}: \theta(t) \in T(\delta)\}<q_{14},
$$

where $q_{14}$ is such that $\theta\left(q_{14}\right)=\widehat{f}^{N_{2}}\left(p_{0}\right)$. This implies that

$$
\max \{t \in \mathbf{R}: \bar{\theta}(t) \in \Delta\}=\max \{t \in \mathbf{R}: \bar{\theta}(t) \in \bar{\delta}\}<q_{14} .
$$

By (51), (52) and by Lemma 2.23 we have that

$$
\bar{f}_{N_{2}}(\bar{p}) \in R(\Delta)
$$

which proves item 3

Finally, we prove Item 4 . As $T(\delta)$ is below $\kappa$, we have the inequality

$$
\begin{aligned}
\max \left\{t \in \mathbf{R}: \bar{\theta}(t) \in \bar{\kappa} \cup \bar{L}\left(\bar{c}_{0}\right) \cup \bar{R}(\bar{\beta})\right\} & =\max \{t \in \mathbf{R}: \bar{\theta}(t) \in \bar{\kappa}\} \\
& <\min \{t \in \mathbf{R}: \bar{\theta}(t) \in \bar{\delta}\} \\
& =\min \{t \in \mathbf{R}: \bar{\theta}(t) \in \Delta\} .
\end{aligned}
$$

By this, as $\operatorname{IN}(\bar{\theta}, \Delta)=1$ (by (51)) and by Lemma 2.23 , we get that the connected set $\bar{\kappa} \cup \bar{L}\left(\bar{c}_{0}\right) \cup$ $\bar{R}(\bar{\beta})$ is contained in $L(\Delta)$. This implies Item 4 .

We may now proceed to the proof of Claim 4.36 .

Proof of Claim 4.36 We will first show that

$$
\bar{f}_{N_{2}} \bar{\eta} \cap \Delta \neq \emptyset .
$$

Recall that by Item 3 from Lemma 4.37, there is $a>0$ such that

$$
\bar{f}_{N_{2}} \bar{\eta}(t) \in L\left(\bar{c}_{1}\right), \quad \text { for } t<a .
$$

By Item 3 of Lemma $4.34 \bar{\beta}$ is a Brouwer curve for $\bar{f}_{N_{2}}$, and therefore

$$
\bar{f}_{N_{2}}(\bar{\eta}(1)) \in \bar{f}_{N_{2}}(\bar{\beta}) \subset R(\bar{\beta}) .
$$


Also, by Item 4 from Lemma 4.38 , we know that

$$
\bar{L}\left(\bar{c}_{0}\right) \cup \bar{R}(\bar{\beta}) \subset L(\Delta),
$$

and therefore, to prove 53 it suffices to show that

$$
\bar{f}_{N_{2}} \bar{\eta} \cap \bar{R}(\Delta) \neq \emptyset .
$$

We suppose that (55) does not hold, and we will find a contradiction. By Item 2 from Lemma 4.38. we know that $\bar{f}_{N_{2}}(\bar{p}) \in \bar{f}_{N_{2}}\left(L\left(\bar{\eta} \cdot \bar{\beta}^{\prime}\right)\right)=L\left(\bar{f}_{N_{2}}\left(\bar{\eta} \cdot \bar{\beta}^{\prime}\right)\right)$. By this and by item 3 from Lemma 4.38 we have that

$$
L\left(\bar{f}_{N_{2}}\left(\bar{\eta} \cdot \bar{\beta}^{\prime}\right)\right) \cap R(\Delta) \neq \emptyset .
$$

As $\bar{\beta}^{\prime} \cap \Delta=\emptyset$, we obtain that, if 55 does not hold, then

$$
L\left(\bar{f}_{N_{2}}\left(\bar{\eta} \cdot \bar{\beta}^{\prime}\right)\right) \supset R(\Delta) .
$$

On the other hand, as $\bar{f}_{N_{2}} \bar{\eta}$ intersects $L\left(\bar{c}_{0}\right)$ and $R(\bar{\beta})$, and by 54 , we have

$$
\bar{f}_{N_{2}} \bar{\eta} \subset L(\Delta) \text {. }
$$

Then, we must have

$$
\max \left\{t \in \mathbf{R}: \bar{\theta}(t) \in \bar{f}_{N_{2}} \bar{\eta}\right\}<\min \{t \in \mathbf{R}: \bar{\theta}(t) \in \Delta\} .
$$

As $\operatorname{IN}\left(\bar{\theta}, \bar{f}_{N_{2}} \bar{\eta} \cdot \bar{\beta}^{\prime}\right)=1$ (by item 1 from Lemma 4.38 , by Lemma 2.23 it holds

$$
R(\Delta) \subset R\left(\bar{f}_{N_{2}}\left(\bar{\eta} \cdot \bar{\beta}^{\prime}\right)\right),
$$

which contradicts (56). This contradiction proves (55), and therefore (53).

We will now see that

$$
\bar{f}_{N_{2}} \bar{\eta} \cap \bar{\delta}_{6}=\bar{f}_{N_{2}} \bar{\eta} \cap \bar{\delta}_{7}=\emptyset .
$$

This, together with 53 , will imply that $\bar{f}_{N_{2}} \bar{\eta}$ intersects $\bar{\delta}$, which proves Claim 4.36

Note that, by item 3 from Lemma $4.34, \bar{\delta}_{1}$ and $\bar{\delta}_{2}$ are Brouwer curves for $\bar{f}_{1}$. If we had that $\bar{f}_{N_{2}} \bar{\eta} \cap \bar{\delta}_{6} \neq \emptyset$, as $\bar{\delta}_{6} \subset \delta_{1}$ this would give

$$
\left(\bar{f}_{1}\right)^{-m} \bar{f}_{N_{2}} \bar{\eta} \cap L\left(\bar{\delta}_{1}\right)=\left(\bar{f}_{1}\right)^{-m+N_{2}} \bar{\eta} \cap L\left(\bar{\delta}_{1}\right) \neq \emptyset \quad \forall m \geq 0 .
$$

On the other hand, by definition $\eta$ is contained in some iterate $\widehat{f^{n}} \tilde{\gamma}, n>0$, of the curve $\tilde{\gamma}$, and $\tilde{\gamma}$ is disjoint from $\delta_{1} \cup \delta_{2}$, which implies

$$
\left(\bar{f}_{1}\right)^{-n} \bar{\eta} \cap \bar{\delta}_{1}=\emptyset .
$$

By $54, L\left(\bar{c}_{0}\right) \subset L(\Delta) \subset R\left(\bar{\delta}_{1}\right)$, and then, by item 3 from Lemma 4.37 we have that $\left(\bar{f}_{1}\right)^{-n} \bar{\eta}(t)$ belongs to $L\left(\bar{c}_{0}\right) \subset R\left(\bar{\delta}_{1}\right)$ for $t$ small. By this and by $(58)$ we have that $\left(\bar{f}_{1}\right)^{-n} \bar{\eta}$ intersects $\bar{\delta}_{1}$, which contradicts $(59)$. Therefore, we must have that $\overline{f_{N_{2}}} \bar{\eta} \cap \bar{\delta}_{6}=\emptyset$.

The same argument shows that $\bar{f}_{N_{2}}(\bar{\eta}) \cap \bar{\delta}_{7}=\emptyset$. This shows 57 and finishes the proof of the claim. 


\subsubsection{Proof of Lemma 4.32}

By definition, if $\widehat{f}^{n}(\widetilde{\gamma})$ has good intersection with $\widehat{f}^{-N_{1}}\left(\beta_{i}\right)$ we have that $\widehat{f}^{n}(\tilde{\gamma})$ contains a subarc $\mu$ which goes from $\hat{f}^{n}(\tilde{\gamma}(0))=\tilde{\gamma}(0)$ to $\widehat{f}^{-N_{1}}\left(\beta_{i}\right)$, which is homotopic wfe $\operatorname{Rel}\left(\widehat{f}^{-N_{1}}\left(F_{i}\right)\right)$ to an arc $\nu$ of the form $\nu=\nu_{1} \cdot \nu_{2} \cdot \nu_{3}$, where $\nu_{1}$ is horizontal, $\nu_{2}$ is vertical, and $\nu_{3}$ is contained $T^{i}(\delta)$ (cf. Definition 4.30 and Fig. 14. Clearly, $\nu_{1} \cdot \nu_{2}$ is homotopic wfe $\operatorname{Rel}\left(\widehat{f}^{-N_{1}}\left(F_{i}\right)\right)$ to the arc $\nu_{1}^{\prime} \cdot \nu_{2}^{\prime}$, where $\nu_{1}^{\prime}$ is straight vertical and $\nu_{2}^{\prime}$ is straight horizontal. If $\nu^{\prime}=\nu_{1}^{\prime} \cdot \nu_{2}^{\prime} \cdot \nu_{3}$, we then have that $\mu$ is homotopic wfe $\operatorname{Rel}\left(\widehat{f}^{-N_{1}}\left(F_{i}\right)\right)$ to $\nu^{\prime}$.

Let $\epsilon$ be the arc $\epsilon(t)=\widehat{f}_{t}(\mu(1)), 0 \leq t \leq N_{1}$, and reparametrize $\epsilon$ so that it is defined in $[0,1]$. By definition of $p_{0}$ in 4.6 .2 we have that $\left\{\widehat{f}_{t}\left(p_{0}\right): t \in\left[-N_{1}, 0\right]\right\}$ is above $\left\{\widehat{f}_{t}\left(\beta_{0}\right): t \in\right.$ $\left.\left[-N_{1}, 0\right]\right\}$, and by item 2 of Claim 4.27 we have that $\hat{f}^{-j\left(N_{1}+N_{2}\right)}\left(p_{j}\right)$ is above $p_{0}$ for all $j>0$. Therefore, by periodicity

$$
\left\{\widehat{f}_{t}\left(\widehat{f}^{-j\left(N_{1}+N_{2}\right)}\left(p_{j}\right)\right): t \in\left[-N_{1}, 0\right]\right\} \text { is above }\left\{\widehat{f}_{t}\left(\beta_{i}\right): t \in\left[-N_{1}, 0\right]\right\} \text { for all } j>i .
$$

Also, the set $\left\{\widehat{f}_{t}\left(\beta_{i}\right): t \in\left[-N_{1}, 0\right]\right\}$ is disjoint from the sets $\operatorname{sing}\left(\beta_{j}\right), \operatorname{sing}\left(T^{j}\left(\delta_{1}\right)\right), \operatorname{sing}\left(T^{j}\left(\widetilde{\delta}_{2}\right)\right)$ for any $j$. By, by 60$\}$ and as $\epsilon \subset\left\{\widehat{f}_{t}\left(\beta_{i}\right): t \in\left[-N_{1}, 0\right]\right\}$ we conclude that

$$
\left\{\widehat{f}_{t}\left(F_{i}\right): t \in\left[-N_{1}, 0\right]\right\} \cap \epsilon=\emptyset .
$$

Similarly, we have

$$
\left\{\widehat{f_{t}}\left(F_{i}\right): t \in\left[-N_{1}, 0\right]\right\} \cap \nu^{\prime}=\emptyset .
$$

By 61 and 62 , Lemma 2.21 gives us that $\nu^{\prime} \cdot \epsilon$ is homotopic wfe $\operatorname{Rel}\left(\widehat{f}^{N_{1}}\left(\widehat{f}^{-N_{1}}\left(F_{i}\right)\right)\right)=\operatorname{Rel}\left(F_{i}\right)$ to $f^{N_{1}} \mu$.

Let $\bar{\nu}_{3}$ be a lift of $\nu_{3}$ to the universal cover $\mathbf{D}$ of $\mathbf{R}^{2} \backslash\left(F_{i} \cap \operatorname{sing}(\widehat{\mathcal{F}})\right)$, let $\bar{\epsilon}$ be the lift of $\epsilon$ to D with $\bar{\epsilon}(0)=\bar{\nu}_{3}(1)$, and let $\bar{\beta}_{i}$ be a lift of $\beta_{i}$ such that $\bar{\epsilon}(1) \in \bar{\beta}_{i}$. Observe that, by Theorem 2.13. $\epsilon$ is homotopic wfe $\operatorname{Rel}(\operatorname{sing}(\widehat{\mathcal{F}}))$ to an arc $\epsilon^{\prime}$ which is positively transverse to $\widehat{\mathcal{F}}$, and then $\bar{\epsilon}(0) \in L\left(\bar{\beta}_{i}\right)$. As $\nu_{3} \cap \beta_{i}=\emptyset$, we have also $\bar{\nu}_{3} \cdot \bar{\epsilon}(0)=\bar{\nu}_{3}(0) \in L\left(\bar{\beta}_{i}\right)$. Note also that the arcs $\epsilon$ and $\nu_{3}$ are contained in the strip

$$
B_{i}=\left\{x \in \mathbf{R}^{2}: x \text { is below } p_{i} \text { and above } \beta_{i} \cup T^{i}\left(\delta_{1} \cup \widetilde{\delta}_{2}\right)\right\},
$$

and that if $\bar{\nu}_{2}^{\prime}$ is the lift of $\nu_{2}^{\prime}$ with $\bar{\nu}_{2}^{\prime}(1)=\bar{\nu}_{3}(0)$, we have $\bar{\nu}_{2}^{\prime}(0) \in L\left(\bar{\beta}_{i}\right)\left(\right.$ as $\left.\nu_{2}^{\prime} \cap \beta_{i}=\emptyset\right)$.

We conclude that $\widehat{f}^{N_{1}} \mu$ is homotopic wfe $\operatorname{Rel}\left(F_{i}\right)$ to the $\operatorname{arc} \nu^{\prime} \cdot \epsilon=\nu_{1}^{\prime} \cdot \nu_{2}^{\prime} \cdot \nu_{3}^{\prime} \cdot \epsilon$, where:

- $\nu_{1}^{\prime}$ is straight vertical, and

- $\nu_{2}^{\prime} \cdot \nu_{3} \cdot \epsilon$ is contained in $B_{i} \backslash F_{i}$ and lifts to the $\operatorname{arc} \bar{\nu}_{2}^{\prime} \cdot \bar{\nu}_{3} \cdot \bar{\epsilon}$, with $\bar{\nu}_{2}^{\prime} \cdot \bar{\nu}_{3} \cdot \bar{\epsilon}(0) \in L\left(\bar{\beta}_{i}\right)$.

This means that $\widehat{f}^{n+N_{1}}(\widetilde{\gamma}) \supset \widehat{f}^{N_{1}} \mu$ satisfies the definition of being well positioned with respect to $p_{i}$, with $\eta=\widehat{f}^{N_{1}} \mu, \kappa_{1}=\nu_{1}^{\prime}$ and $\kappa_{2}=\nu_{2}^{\prime} \cdot \nu_{3} \cdot \epsilon$, which proves Lemma 4.32

\subsection{Construction of the $\operatorname{arc} \delta$; proof of Lemma 4.25.}

We begin by recalling the statement of the lemma.

Lemma. There exists an arc $\delta$ such that:

1. $\operatorname{int}(\delta) \subset \widehat{f}^{-N_{1}} \beta$,

2. $\delta(0) \in \mathcal{C}_{-2}, \delta(1) \in \mathcal{C}_{0}, \operatorname{int}(\delta) \subset R\left(\mathcal{C}_{-2}\right) \cap L\left(\mathcal{C}_{0}\right)$, 
3. $\delta$ leaves a leaf $\delta_{1} \subset \mathcal{C}_{-2}$ on $t=0$ by the right (cf. Definition 2.18),

4. one of the following holds:

(a) $\delta(1) \in \operatorname{sing}(\widehat{\mathcal{F}}) \cap \mathcal{C}_{0}$.

(b) $\delta$ arrives in a leaf $\delta_{2} \subset \mathcal{C}_{0}$ on $t=1$ by the right (see Fig. 11).

We also recall from Notation 4.26 our definition of $\widetilde{\delta}_{2}=\emptyset$ in case item $4(a)$ holds, and $\widetilde{\delta}_{2}=\delta_{2}$ in case item $4(b)$ holds.

4.7.1 Case that either $\alpha(\gamma)$ or $\omega(\gamma)$ is a singularity of $\widehat{\mathcal{F}}$, and either $\alpha(\beta)$ or $\omega(\beta)$ is a singularity of $\widehat{\mathcal{F}}$.

We will assume that $\omega(\gamma)$ and $\omega(\beta)$ are singularities, $\omega(\gamma)=\left\{s_{1}\right\}$ and $\omega(\beta)=\left\{s_{2}\right\}$, for some $s_{1}, s_{2} \in \operatorname{sing}(\widehat{\mathcal{F}})$. The complementary case that (at least) one of the sets $\alpha(\gamma)$ and $\alpha(\beta)$ is a singularity, and (at least) one of the sets $\omega(\gamma)$ and $\omega(\beta)$ is not a singularity will follow from the same argument.

Definition 4.39. We say that an arc $\eta$ has a removable intersection in $t \in(0,1)$ with $\mathcal{C}_{-2}$ if there exists a leaf $\epsilon$ of $\widehat{\mathcal{F}}$ contained in $\mathcal{C}_{-2}$ and $t^{\prime} \in(t, 1)$ such that:

- $\eta(t), \eta\left(t^{\prime}\right) \in \epsilon$, and

- $\left.\eta\right|_{\left[t, t^{\prime}\right]}$ is homotopic wfe $\operatorname{Rel}(\operatorname{sing}(\widehat{\mathcal{F}}))$ to the subarc of $\epsilon$ going from $\eta(t)$ to $\eta\left(t^{\prime}\right)$.

We call the interval $\left[t, t^{\prime}\right]$ a removable interval for $\eta$. Also, we denote by $U_{\eta} \subset(0,1)$ the union of the interiors of every removable interval for $\eta$.

Extend the leaves $\gamma, \beta:(0,1) \rightarrow \mathbf{R}^{2}$ to $(0,1]$, so that $\gamma(1)=s_{1}$ and $\beta(1)=s_{2}$. Recall that by definition, $\beta$ is the arc that contains the last point of intersection of $\widehat{f}^{N_{1}} \gamma$ with $\mathcal{C}_{0}$. That is, $\beta$ contains the point $z=\widehat{f}^{N_{1}} \gamma\left(t_{1}\right)$, where

$$
t_{1}=\max \left\{t \in(0,1): \widehat{f}^{N_{1}} \gamma(t)=\mathcal{C}_{0}\right\} .
$$

Let $\beta^{1} \subset \beta$ be the subarc of $\beta$ going from $z$ to $\beta(1)=s_{2}$ (see Fig. 19). Consider the set $U_{\widehat{f}^{-N_{1} \beta^{1}}} \subset(0,1)$ from Definition 4.39, which is the union of the interiors of the removable intervals for $\widehat{f}^{-N_{1}} \beta^{1}$. There exist two possibilities:

Case 1: For every $t \in(0,1)$ such that $\widehat{f}^{-N_{1}} \beta^{1}(t) \in \mathcal{C}_{0}$, it holds $t \in U_{\widehat{f}^{-N_{1}} \beta^{1}}$.

In this case, let

$$
t_{3}=\max \left\{t \in(0,1): \widehat{f}^{-N_{1}} \beta^{1}(t) \in \mathcal{C}_{-2}\right\},
$$

and define $\delta_{1} \subset \mathcal{C}_{-2}$ as the leaf of $\widehat{\mathcal{F}}$ containing $\widehat{f}^{-N_{1}} \beta\left(t_{3}\right)$. Then, $\widehat{f}^{-N_{1}} \beta^{1}$ must leave $\delta_{1}$ in $t_{3}$ by the right; otherwise, it would leave by the left, and by Lemma 2.19 we would have

$$
\beta^{1} \cap \mathcal{C}_{-2}=\widehat{f}^{N_{1}}\left(\widehat{f}^{-N_{1}} \beta^{1}\right) \cap \mathcal{C}_{-2} \supset \widehat{f}^{N_{1}}\left(\widehat{f}^{-N_{1}} \beta^{1}\right) \cap \delta_{1} \neq \emptyset,
$$

which contradicts the fact that $\beta^{1} \subset \mathcal{C}_{0}$.

Also, $\left.\widehat{f}^{-N_{1}} \beta^{1}\right|_{\left(t_{3}, 1\right)} \subset R\left(\mathcal{C}_{-2}\right) \cap L\left(\mathcal{C}_{0}\right)$. To see this, note that, by definition of $t_{3}$, if $t \in\left(t_{3}, 1\right)$ is such that $\widehat{f}^{-N_{1}} \beta^{1}(t) \in \mathcal{C}_{0}$, then $t$ must be contained in $(0,1) \backslash U_{\widehat{f}-N_{1}} \beta^{1}$, which is excluded from Case 1.

Define then

$$
\delta=\left.\widehat{f}^{-N_{1}} \beta^{1}\right|_{\left[t_{3}, 1\right]}
$$


and reparametrize $\delta$ to be defined in $[0,1]$. By the above, $\delta$ satisfies items 1 to 3 and $4($ a) of the lemma.

Case 2: There exists $t \in(0,1)$ such that $\widehat{f}^{-N_{1}} \beta^{1}(t) \in \mathcal{C}_{0}$, and $t \notin U_{\widehat{f}^{-N_{1}} \beta^{1}}$.

Let $x=\widehat{f}^{-N_{1}} \beta^{1}\left(t_{4}\right)$, where

$$
t_{4}=\min \left\{t \in(0,1) \backslash U_{\widehat{f}-N_{1} \beta^{1}}: \widehat{f}^{-N_{1}} \beta^{1}(t) \in \mathcal{C}_{0}\right\} .
$$

Define

$$
\beta^{2}=\left.\widehat{f}^{-N_{1}} \beta^{1}\right|_{\left[0, t_{4}\right]}
$$

(reparametrized to $[0,1]$ ), and let $\delta_{2}$ be the leaf of $\widehat{\mathcal{F}}$ contained in $\mathcal{C}_{0}$ and containing $x=\beta^{2}(1)$. By definition, $\beta^{2}$ is homotopic wfe $\operatorname{Rel}(\operatorname{sing}(\widehat{\mathcal{F}}))$ to an $\operatorname{arc}$ whose first intersection with $\mathcal{C}_{0}$ is the endpoint $x=\beta^{2}(1) \in \delta_{2}$.

Claim 4.40. $\beta^{2}$ arrives in $\delta_{2}$ in $t=1$ by the right.

Proof. Let $\tau_{1} \subset \gamma$ be the arc going from $\gamma(1)=s_{1}$ to $\beta^{2}(0)=\widehat{f}^{-N_{1}} \beta^{1}(0)$. Suppose that $\beta^{2}$ does not arrive in $\delta_{2}$ in 1 by the right. Then it arrives by the left, and then by Lemma 2.19 we have that

$$
\widehat{f}^{N_{1}}\left(\tau_{1} \cdot \beta^{2}\right) \cap \delta_{2} \neq \emptyset .
$$

By construction of $\beta^{1}$ and $\beta^{2}$, we have that

$$
\widehat{f}^{N_{1}}\left(\tau_{1}\right) \cap \mathcal{C}_{0}=\left\{\widehat{f}^{N_{1}} \tau_{1}(1)\right\}=\left\{\widehat{f}^{N_{1}} \beta^{2}(0)\right\} \subset \widehat{f}^{N_{1}} \beta^{2} \cap \mathcal{C}_{0} .
$$

By this, and as $\widehat{f}^{N_{1}}\left(\beta^{2}\right) \subset \beta,(64)$ implies that

$$
\delta_{2}=\beta \text {. }
$$

We will see that this yields a contradiction, and therefore $\beta^{2}$ must arrive in $\delta_{2}$ in 1 by the right.

Let $\bar{\beta}$ be a lift of $\beta$ to the universal cover of $\mathbf{R}^{2} \backslash \operatorname{sing}(\beta)$. Let $\bar{\beta}^{2}$ be the lift of $\beta^{2}$ such that $\bar{\beta}^{2}(1) \in \bar{\beta}$, and let $\bar{\tau}_{1}$ be the lift of $\tau_{1}$ such that $\bar{\tau}_{1}(1)=\bar{\beta}^{2}(0)$. By construction, $\beta^{2}$ is homotopic wfe $\operatorname{Rel}(\operatorname{sing}(\widehat{\mathcal{F}}))$ to an arc whose only intersection with $\mathcal{C}_{0}$ is in $\beta^{2}(1) \in \beta$. Therefore, by our assumption that $\beta^{2}$ arrives in $\delta_{2}=\beta$ in 1 by the left, we have that

$$
\bar{\tau}_{1} \cdot \bar{\beta}^{2}(0) \in L(\bar{\beta}) .
$$

If $\bar{f}$ is the canonical lift of $\widehat{f}, \bar{\beta}$ is a Brouwer curve for $\bar{f}$, and therefore by Lemma $2.19 \bar{f}^{N_{1}}\left(\bar{\tau}_{1}\right.$. $\left.\bar{\beta}^{2}\right) \cap \bar{\beta} \neq \emptyset$. By 65$)$, this yields

$$
\bar{f}^{N_{1}} \bar{\beta}^{2} \cap \bar{\beta} \supset \bar{f}^{N_{1}}\left(\bar{\tau}_{1} \cdot \bar{\beta}^{2}\right) \cap \bar{\beta} \supset\left\{\widehat{f}^{N_{1}} \bar{\beta}^{2}(0)\right\} \neq \emptyset,
$$

and as $\beta^{2} \subset \widehat{f}^{-N_{1}} \beta$, we must have

$$
\bar{f}^{N_{1}} \bar{\beta}^{2} \subset \bar{\beta} .
$$

As $\bar{\beta}^{2}(1) \in \bar{\beta}$, we have that $\bar{f}^{N_{1}} \bar{\beta} \cap \bar{\beta} \neq \emptyset$, which contradicts the fact that $\bar{\beta}$ is a Brouwer curve for $\bar{f}$. This is the sought contradiction, and this finishes the proof of the claim.

Let $y=\beta^{2}\left(t_{5}\right)$, where

$$
t_{5}=\max \left\{t \in(0,1): \beta^{2}(t) \in \mathcal{C}_{-2}\right\},
$$

and let $t_{6} \in\left[0, t_{4}\right]$ be such that $\widehat{f}^{-N_{1}} \beta^{1}\left(t_{6}\right)=\beta^{2}\left(t_{5}\right)$.

Define $\delta_{1}$ as the leaf of $\widehat{\mathcal{F}}$ contained in $\mathcal{C}_{-2}$ which contains $y$. 


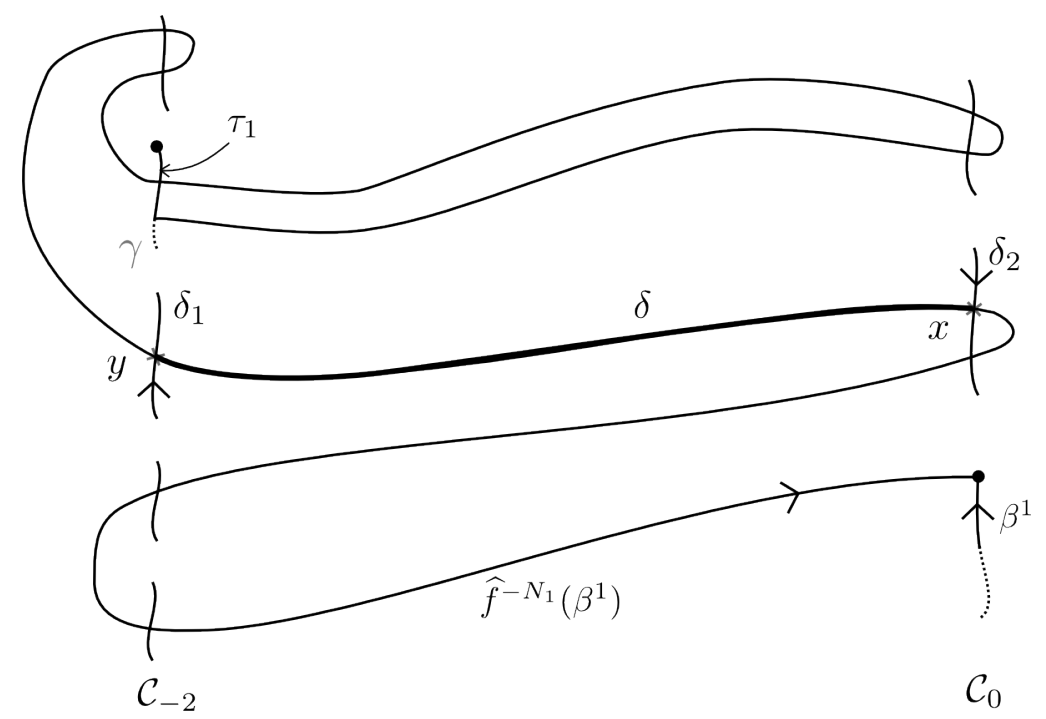

Figure 19: An illustration of the construction of $\delta$, in the case that $\omega(\gamma)$ and $\omega(\beta)$ are singularities.

Claim 4.41. $\widehat{f}^{-N_{1}} \beta^{1}$ leaves $\delta_{1}$ in $t_{6}$ by the right.

Before proving this claim we see how it implies Lemma 4.25 in Case 2. Define $\delta$ as the subarc of $\widehat{f}^{-N_{1}} \beta$ going from $y$ to $x$. As $\delta \subset \widehat{f}^{-N_{1}} \beta, \delta$ satisfies item 1 from the lemma. By definition of $x$ and $y$, int $(\delta) \subset R\left(\mathcal{C}_{-2}\right) \cap L\left(\mathcal{C}_{0}\right)$, and then $\delta$ satisfies item 2. By Claim 4.40 $\delta$ arrives in the leaf $\delta_{2} \subset \mathcal{C}_{0}$ in $t=1$ by the right, and by Claim $4.41 \delta$ leaves the leaf $\delta_{1} \subset \mathcal{C}_{-2}$ in $t=0$ by the right, and then $\delta$ satisfies items 3 and 4(b) of the lemma. This concludes the proof.

We now give the proof of Claim 4.41

Proof of Claim 4.41 If $\widehat{f}^{-N_{1}} \beta^{1}$ does not leave $\delta_{1}$ in $t_{6}$ by the right, it leaves by the left. We will show that this implies a contradiction.

Consider the set $U_{\widehat{f}^{-N_{1} \beta^{1}}} \subset[0,1]$, which is a (possibly empty) union of open intervals $I_{i}$ (cf. Definition 4.39). We know that, for each $i,\left.\widehat{f}^{-N_{1}} \beta^{1}\right|_{I_{i}}$ is homotopic wfe $\operatorname{Rel}(\operatorname{sing}(\widehat{\mathcal{F}}))$ to an arc $\epsilon_{i} \subset \mathcal{C}_{-2}$ contained in a leaf of $\widehat{\mathcal{F}}$.

Consider the canonical $\bar{f}$ of $\widehat{f}$ to the universal cover of $\mathbf{R}^{2} \backslash \operatorname{sing}\left(\delta_{1}\right)$, let $\bar{\delta}_{1}$ be any lift of $\delta_{1}$, and let $\bar{\beta}$ be a lift of $\widehat{f}^{-N_{1}} \beta^{1}$ such that $\bar{\beta}\left(t_{6}\right) \in \bar{\delta}_{1}$. By definition, $t_{6}$ is not a removable intersection for $\widehat{f}^{-N_{1}} \beta^{1}$. By this, and as we are assuming that $\widehat{f}^{-N_{1}} \beta^{1}$ leaves $\delta_{1}$ in $t_{6}$ by the left, we have that $\bar{\beta}$ leaves $\bar{\delta}_{1}$ in $t_{6}$ by the left and

$$
\bar{\beta}(1) \in L\left(\bar{\delta}_{1}\right) .
$$

By Lemma 2.17, this yields $\bar{f}^{N_{1}} \bar{\beta} \cap \bar{\delta}_{1} \neq \emptyset$, and therefore

$$
\beta^{1} \cap \mathcal{C}_{-2}=\widehat{f}^{N_{1}}\left(\widehat{f}^{-N_{1}} \beta^{1}\right) \cap \mathcal{C}_{-2} \supset \widehat{f}^{N_{1}}\left(\widehat{f}^{-N_{1}} \beta^{1}\right) \cap \delta_{1} \neq \emptyset,
$$

which is the desired contradiction, as $\beta^{1} \subset \beta \subset \mathcal{C}_{0}$. 


\subsubsection{Case that none of the sets $\alpha(\gamma), \omega(\gamma), \alpha(\beta)$, and $\omega(\beta)$ consist of a singularity.}

We recall the definition of $\beta$ from Section 4.6.2, for the case that neither $\omega(\gamma)$ nor $\omega(\beta)$ consist of a singularity.

We first defined a straight horizontal arc $\lambda_{1}$ going leftwards from a singularity $s \in \operatorname{Fill}(\omega(\gamma))$ to a point of $\widehat{f}^{N_{1}}(\gamma)$ sufficiently close to $\widehat{f}^{N_{1}}(\omega(\gamma))$ so that $\widehat{f}^{-N_{1}}\left(\lambda_{1}\right) \cap \mathcal{C}_{0}=\emptyset$. Then, we defined $\lambda_{2}$ as a subarc of $\widehat{f}^{N_{1}} \gamma$ going from $\lambda_{1}(1)$ to a point $z \in \widehat{f}^{N_{1}} \gamma \cap \mathcal{C}_{0}$ such that $\left.\lambda_{2}\right|_{[0,1)} \subset L\left(\mathcal{C}_{0}\right)$. Finally, we set $\lambda=\lambda_{1} \cdot \lambda_{2}$, and defined $\beta$ as the leaf of $\widehat{\mathcal{F}}$ that contains $\lambda(1)$.

Claim 4.42. The $\operatorname{arc} \lambda=\lambda_{1} \cdot \lambda_{2}$ arrives in $\beta$ in $t=1$ by the left.

Proof. Suppose that $\lambda$ does not arrive in $\beta$ in 1 by the left. Then $\lambda$ must arrive by the right, and by Lemma 2.19 we have that

$$
\widehat{f}^{-N_{1}} \lambda \cap \mathcal{C}_{0} \supset \widehat{f}^{-N_{1}} \lambda \cap \beta \neq \emptyset,
$$

which contradicts the fact that $\widehat{f}^{-N_{1}} \lambda_{1} \cap \mathcal{C}_{0}=\emptyset$. Therefore, we must have that $\lambda$ arrives in $\beta$ by the left.

Lemma 4.43. There exist arcs $\beta^{1}$ and $\beta^{2}$ such that:

1. $\beta^{1}(0) \in \operatorname{sing}(\widehat{\mathcal{F}}) \cap \operatorname{Fill}(\omega(\beta))$,

2. $\left(\beta^{1} \cup \widehat{f}^{N_{1}} \beta^{1}\right) \cap \mathcal{C}_{-2}=\emptyset$,

3. $\beta^{2}(0)=\beta^{1}(1), \beta^{2} \subset \widehat{f}^{-N_{1}} \beta$ and $\beta^{2}(1)=\widehat{f}^{-N_{1}} \lambda(1)$,

4. there exists $t_{1} \in[0,1)$ such that $\beta^{2}\left(t_{1}\right) \in \beta$ and $\beta^{2}(t) \in R\left(\mathcal{C}_{-2}\right)$ for all $t \in\left[0, t_{1}\right]$.

We postpone the proof of this lemma to the end of this section. Define

$$
\Gamma=\widehat{f}^{-N_{1}} \lambda \cdot\left(\beta^{2}\right)^{-1} \cdot\left(\beta^{1}\right)^{-1},
$$

(cf. Fig. 200. Consider the set $U_{\Gamma}$, which is the (possibly empty) union of removable intervals for $\Gamma$ (cf. Definition 4.39). Let $t_{1}$ be as in item 4 from Lemma 4.43 , and let $t_{1}^{\prime}$ be such that $\Gamma\left(t_{1}^{\prime}\right)=\beta^{2}\left(t_{1}\right) \in \beta$. As $\beta^{1} \cap \mathcal{C}_{-2}=\emptyset$ (by item 2 of such lemma), it follows that $t_{1}^{\prime}$ is not contained in $U_{\Gamma}$.

We may then define $x=\Gamma\left(t_{2}\right)$, where

$$
t_{2}=\min \left\{t \in(0,1) \backslash U_{\Gamma}: \Gamma(t) \in \mathcal{C}_{0}\right\} .
$$

Observe that as $\Gamma\left(t_{1}^{\prime}\right)=\beta^{2}\left(t_{1}\right) \in \beta$,

$$
\left.\Gamma\right|_{\left[0, t_{2}\right]} \subset\left(\widehat{f}^{-N_{1}} \lambda\right) \cdot\left(\beta^{2}\right)^{-1} .
$$

Let $\delta_{2} \subset \mathcal{C}_{0}$ be the leaf of $\widehat{\mathcal{F}}$ that contains $x$.

Claim 4.44. $\Gamma$ arrives in $\delta_{2}$ in $t_{2}$ by the right.

Before proving this claim, we use it to construct the $\operatorname{arc} \delta$. Let $y=\Gamma\left(t_{3}\right)$, where

$$
t_{3}=\max \left\{t \in\left(0, t_{2}\right): \Gamma(t) \in \mathcal{C}_{-2}\right\},
$$

and define

$$
\delta=\Gamma_{\left[t_{3}, t_{2}\right]},
$$

(reparametrized to $[0,1])$. Let $\delta_{1}$ be the leaf of $\widehat{\mathcal{F}}$ that contains $y$.

By definition of $t_{3}$ we have that $\operatorname{int}(\delta) \subset R\left(\mathcal{C}_{-2}\right) \cap L\left(\mathcal{C}_{0}\right)$ and $\delta \subset \beta^{2} \subset \widehat{f}^{-N_{1}} \beta$, and then $\delta$ satisfies items 1 and 2 from the Lemma 4.25. By Claim $4.44 \delta$ arrives in $\delta_{2}$ in 1 by the right and then $\delta$ satisfies item 4 (b). Finally, the claim below will give us that $\delta$ satisfies item 3 . 


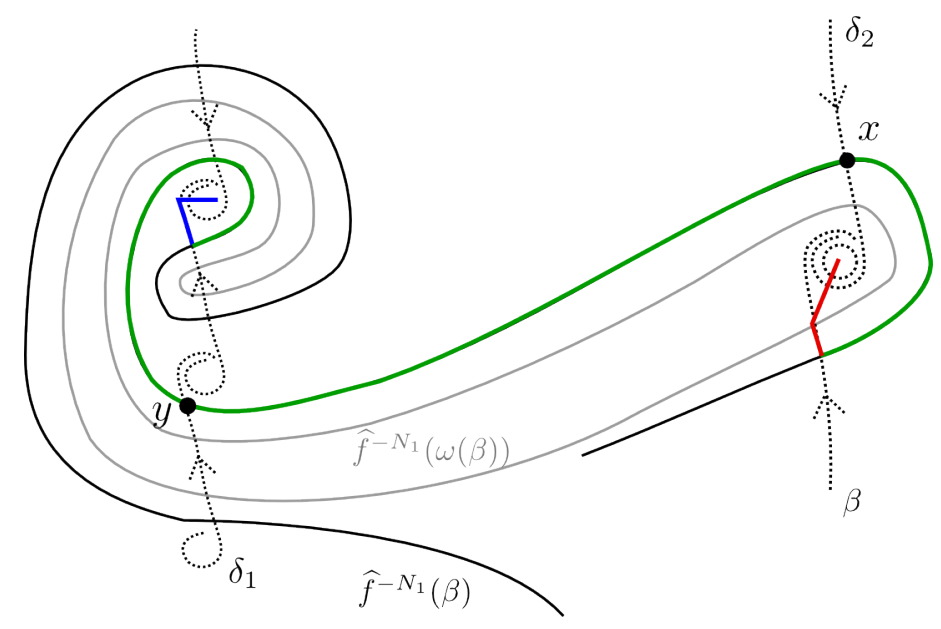

Figure 20: An illustration of the $\operatorname{arc} \Gamma$. In blue the $\operatorname{arc} \widehat{f}^{-N_{1}} \lambda$, in green $\beta^{2}$ and in red $\beta^{1}$.

Claim 4.45. $\delta$ leaves $\delta_{1}$ in $t=0$ by the right.

We proceed to the proof of Claims 4.44 and 4.45 .

Proof of Claim 4.44 If the claim does not hold, then $\Gamma$ arrives in $\delta_{2}$ in $t_{2}$ by the left. By definition of $t_{2},\left.\Gamma\right|_{\left[0, t_{2}\right]}$ is homotopic wfe Rel $\operatorname{sing}(\widehat{\mathcal{F}})$ to an arc whose first and only intersection with $\mathcal{C}_{0}$ is in $x$, and such intersection is by the left. Then, by Lemma 2.19 we have that there is $t \in\left[0, t_{2}\right]$ such that $\widehat{f}^{N_{1}} \Gamma(t) \in \delta_{2}$. By 67 and by definition of $\lambda$, we have that

$$
\widehat{f}^{N_{1}}\left(\left.\Gamma\right|_{\left[0, t_{2}\right]}\right) \cap \mathcal{C}_{0} \subset \widehat{f}^{N_{1}}\left(\widehat{f}^{-N_{1}}(\lambda) \cdot\left(\beta^{2}\right)^{-1}\right) \cap \mathcal{C}_{0} \subset \beta .
$$

This yields $\delta_{2}=\beta$. We will see that this leads to a contradiction.

Consider the canonical lift $\bar{f}$ of $\widehat{f}$ to the universal cover of $\mathbf{R}^{2} \backslash \operatorname{sing}(\beta)$. Fix a lift $\bar{\beta}$ of $\beta$, and consider the lift $\bar{\Gamma}$, of $\left.\Gamma\right|_{[0,1)}$ such that $\bar{\Gamma}\left(t_{2}\right) \in \bar{\beta}$. As $\Gamma_{\left[0, t_{2}\right]}$ is homotopic wfe $\operatorname{Rel}(\operatorname{sing}(\widehat{\mathcal{F}}))$ to an arc that intersects $\delta_{2}$ only in $x=\Gamma\left(t_{2}\right)$, and by our assumption that $\Gamma$ arrives in $\delta_{2}$ in $x$ by the left, we have

$$
\bar{\Gamma}(0) \in L(\bar{\beta}),
$$

and then by Lemma 2.17 we have that $\left.\bar{f}^{N_{1}} \bar{\Gamma}\right|_{\left[0, t_{2}\right]} \cap \bar{\beta} \neq \emptyset$. Also, by 67 and by definition of $\lambda$, $\left.\widehat{f}^{N_{1}} \Gamma\right|_{\left[0, t_{2}\right]} \cap \beta \subset \widehat{f}^{N_{1}}\left(\left(\beta^{2}\right)^{-1}\right) \subset \beta$. Then, we must have that, if $\bar{\beta}^{2}$ is the lift of $\beta^{2}$ contained in $\bar{\Gamma}$

$$
\bar{f}^{N_{1}} \bar{\beta}^{2} \subset \bar{\beta} .
$$

As $\bar{\Gamma}\left(t_{2}\right) \in \bar{\beta}^{2}$ and $\bar{\Gamma}\left(t_{2}\right) \in \bar{\beta}$, this contradicts the fact that $\bar{\beta}$ is a Brouwer curve for $\bar{f}$. This contradiction proves the claim.

Proof of Claim 4.45 We suppose on the contrary that $\delta$ leaves $\delta_{1}$ in $t=0$ by the left, and we will find a contradiction. Consider the canonical lift $\bar{f}$ of $\widehat{f}$ to the universal cover of $\mathbf{R}^{2} \backslash \operatorname{sing}\left(\delta_{1}\right)$. Fix a lift $\bar{\delta}_{1}$ of $\delta_{1}$, and let $\bar{\Gamma}$ be the lift of $\left.\Gamma\right|_{(0,1]}$ such that $\bar{\Gamma}\left(t_{3}\right) \in \bar{\delta}_{1}$. Note that, as $t_{2} \notin U_{\Gamma}, t_{3}$ is not a removable intersection for $\Gamma$. By this and as $\Gamma$ leaves $\delta_{1}$ in $t_{3}$ by the left, we must have that

$$
\bar{\Gamma}(1) \in L\left(\bar{\delta}_{1}\right) .
$$


By Lemma 2.17 we have that $\left.\bar{f}^{N_{1}} \bar{\Gamma}\right|_{\left[t_{3}, 1\right]} \cap \bar{\delta}_{1} \neq \emptyset$, and by item 2 from Lemma $4.43 \widehat{f}^{N_{1}} \beta^{1} \cap \mathcal{C}_{-2}=$ $\emptyset$. Thus

$$
\widehat{f}^{N_{1}} \beta_{2} \cap \mathcal{C}_{-2} \supset \widehat{f}^{N_{1}}\left(\beta^{2} \cdot \beta^{1}\right) \cap \mathcal{C}_{-2} \supset \widehat{f}^{N_{1}}\left(\left.\Gamma\right|_{\left[t_{3}, 1\right]}\right) \cap \delta_{1} \neq \emptyset,
$$

which contradicts the fact that $\widehat{f}^{N_{1}} \beta^{2} \subset \beta \subset \mathcal{C}_{0}$. This is the sought contradiction, and this proves the claim.

Proof of Lemma 4.43 We have four possibilities.

Case 1: $\omega(\beta)$ contains no singularities, and $\widehat{f}^{-1} \omega(\beta) \subset \operatorname{Fill}(\omega(\beta))$.

Let $\beta_{1}^{1}$ be an arc contained in Fill $\left(\widehat{f}^{-N_{1}} \omega(\beta)\right)$, going from a singularity up to a point $p \in \widehat{f}^{-1} \omega(\beta)$. Define $\beta_{2}^{1}$ as an arc going from $p$ to a point of $\widehat{f}^{-N_{1}} \beta$, positively transverse to $\widehat{f}^{-N_{1}} \beta$ and suficiently small in order that $\beta_{2}^{1} \subset \operatorname{Fill}(\omega(\beta))$ and $\beta_{2}^{1} \cup \widehat{f}^{N_{1}} \beta_{2}^{1} \cap \mathcal{C}_{-2}=\emptyset$ (see Fig. 21.

Define then $\beta^{1}=\beta_{1}^{1} \cdot \beta_{2}^{1}$, and note that

$$
\left(\beta^{1} \cup \widehat{f}^{N_{1}} \beta^{1}\right) \cap \mathcal{C}_{-2}=\emptyset,
$$

and then $\beta^{1}$ satisfies items 1 and 2 .

Define now $\beta^{2}$ as the subarc of $\widehat{f}^{-N_{1}}(\beta)$ going from $\beta^{1}(1)$ to $\widehat{f}^{-N_{1}} \lambda(1) \in \mathcal{C}_{-2}$. The arc $\beta^{2}$ satisfies then item (3) from the lemma. By last, we see that $\beta^{2}$ satisfies item (4).

Let

$$
t_{0}=\max \left\{t \in[0,1]: \beta^{2}(t) \in \omega(\beta) \text { and } \beta^{2}(s) \in \operatorname{Fill}(\omega(\beta)) \text { for all } s \in[0, t]\right\} .
$$

Then, there exist points $t \in\left(t_{0}, 1\right)$ arbitrarily close to $t_{0}$ such that $\beta^{2}(t) \notin \operatorname{Fill}(\omega(\beta))$, and such that $\beta^{2}(t) \in \beta$ (see Fig. 21). Choose then $t_{1} \in\left(t_{0}, 1\right)$ close enough to $t_{0}$ so

$$
\left.\beta^{2}\right|_{\left[0, t_{1}\right]} \subset R\left(\mathcal{C}_{-2}\right) .
$$

The point $t_{1}$ and the arc $\beta^{2}$ satisfy then item (4), as we wanted.
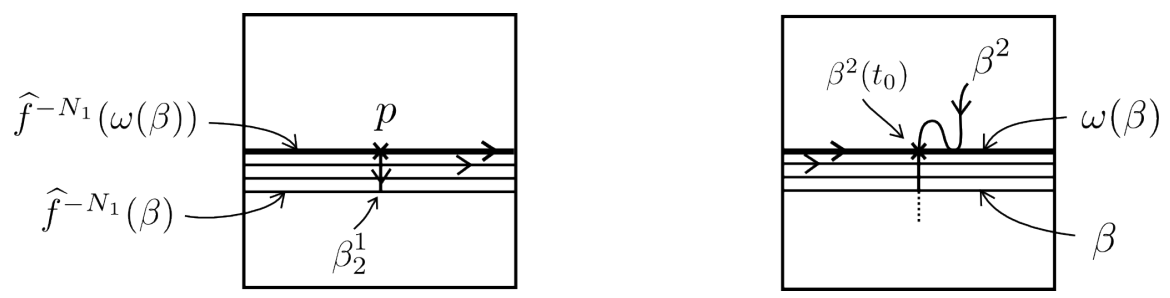

Figure 21: Illustration of Case 1. Left: the $\operatorname{arc} \beta_{2}^{1}$. Right: the $\operatorname{arc} \beta^{2}$.

Case 2: $\omega(\beta)$ contains singularities and $\widehat{f}^{-1} \omega(\beta) \subset \operatorname{Fill}(\omega(\beta))$.

By Theorem 2.22 we know that in this case, $\omega(\beta)$ is a generalized cycle of connections of $\widehat{\mathcal{F}}$. Let $\epsilon$ be a leaf of $\widehat{\mathcal{F}}$ contained in $\widehat{f}^{-N_{1}}(\omega(\beta))$, and think of $\epsilon$ as an arc whose endpoints are singularities (both endpoints may coincide, and in this case $\epsilon$ is a loop containing one singularity). Let $p \in \operatorname{int}(\epsilon)$, and let $\beta_{1}^{1}$ be the subarc of $\epsilon$ going from $\epsilon(0)$ to $p$ (see Fig. 22. If $p$ is close enough to an endpoint of $\epsilon$, then there is an $\operatorname{arc} \beta_{2}^{1}$ going from $p$ to a point of $\widehat{f}^{-N_{1}} \beta \cap \operatorname{Fill}(\omega(\beta))$, with $\beta_{2}^{1}$ sufficiently small in order that

$$
\widehat{f}^{N_{1}} \beta_{2}^{1} \cap \mathcal{C}_{-2}=\emptyset
$$


Let $\beta^{1}=\beta_{1}^{1} \cdot \beta_{2}^{1}$. The arc $\beta^{1}$ satisies items 1 and 2 .

Define $\beta^{2}$ as the subarc of $\widehat{f}^{-N_{1}} \beta$ going from $\beta^{1}(1)$ to $\widehat{f}^{-N_{1}} \lambda(1) \in \mathcal{C}_{-2}$. The arc $\beta^{2}$ therefore satisfies item (3) from the lemma. By the same argument from Case 1 one can show that $\beta^{2}$ satisfies item (4).
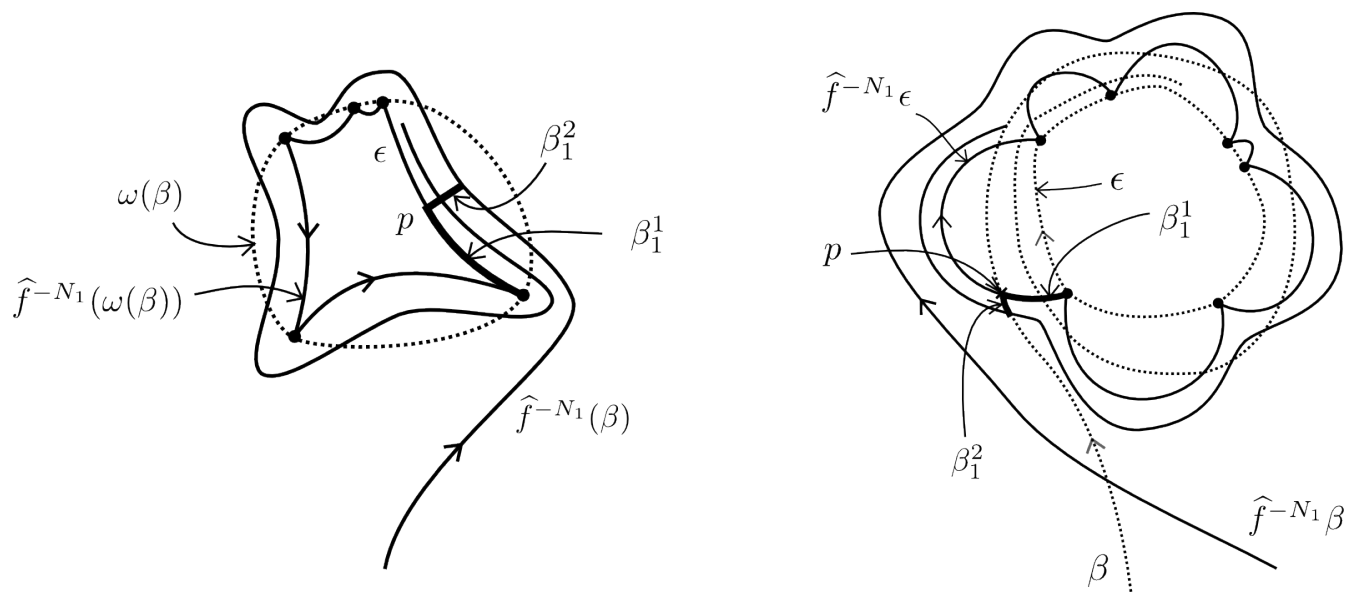

Figure 22: Cases where $\omega(\beta)$ contains singularities. Left: Case $2, \widehat{f}^{-1}(\omega(\beta)) \subset \operatorname{Fill}(\omega(\beta))$. Right: Case $3, \widehat{f}^{-1}(\omega(\beta)) \supset \operatorname{Fill}(\omega(\beta))$.

Case 3: $\omega(\beta)$ contains singularities and $\operatorname{Fill}\left(\hat{f}^{-1}(\omega(\beta)) \supset \omega(\beta)\right.$.

Let $\epsilon$ be a leaf of $\widehat{\mathcal{F}}$ contained in $\omega(\beta)$. Think of $\epsilon$ as an arc whose endpoints $\epsilon(0), \epsilon(1)$ are singularities (both endpoints may coincide). There exist points $p$ of $\beta$ arbitrarily close to $\epsilon(0)$ and such that $\beta$ arrives in $\widehat{f}^{-N_{1}} \epsilon$ in $p$ by the left (see Fig. 22). Let $\beta_{1}^{1}$ be a subarc of $\widehat{f}^{-N_{1}} \epsilon$ going from $\widehat{f}^{-N_{1}} \epsilon(0)$ to one of such points $p$ sufficiently close to $\epsilon(0)$ such that $\beta_{1}^{1} \cap \mathcal{C}_{-2}=\emptyset$. Let $\beta_{2}^{1}$ be a subarc of $\beta$ going from $p$ to a point in $\widehat{f}^{-N_{1}} \beta$, with $\beta_{2}^{1}$ sufficiently small so that

$$
\beta_{2}^{1} \cup \widehat{f}^{N_{1}} \beta_{2}^{1} \cap \mathcal{C}_{-2}=\emptyset .
$$

Let $\beta^{1}=\beta_{1}^{1} \cdot \beta_{2}^{1}$. The arc $\beta^{1}$ satisfies then items 1 and 2 .

Define $\beta^{2}$ be the subarc of $\widehat{f}^{-N_{1}}(\beta)$ going from $\beta^{1}(1)$ to $\widehat{f}^{-N_{1}} \lambda(1) \in \mathcal{C}_{-2}$. The arc $\beta^{2}$ satisfies then item (3) from the lemma. Letting $t_{1}=0$, we clearly have that $t_{1}$ and $\beta^{2}$ satisfy item (4).

Case 4: $\omega(\beta)$ has no singularities and $\operatorname{Fill}\left(\hat{f}^{-1}(\omega(\beta))\right) \supset \omega(\beta)$.

Let $\ell$ be a straight vertical line such that $\mathcal{C}_{-2} \subset L(\ell)$ and $\mathcal{C}_{0} \subset R(\ell)$. Let $D$ be the connected component of $\widehat{f}^{-N_{1}}(\operatorname{Fill}(\omega(\beta))) \cap R(\ell)$ that contains $\omega(\beta)$ (see Fig. 23).

Claim 4.46. $\widehat{f}^{-N_{1}}(\operatorname{Fill}(\omega(\beta)))$ does not contain $\beta$.

With this claim we have that there exists $t_{0} \in[0,1]$ such that $\beta(t) \in D$ for all $t \in\left[t_{0}, 1\right]$. Let $p=\beta\left(t_{0}\right) \in \partial D \cap \widehat{f}^{-N_{1}}(\omega(\beta))$. Then, $\beta$ arrives in $\widehat{f}^{-N_{1}}(\omega(\beta))$ in $p$ by the left. Let $\beta_{1}^{1}$ be an arc contained in $D$ going from a singularity of $\widehat{\mathcal{F}}$ in the interior of $D$ to the point $p$. Let $\beta_{2}^{1}$ be a subarc of $\beta$ going from $p$ to a point of $\widehat{f}^{-N_{1}} \beta$, with $\beta_{2}^{1}$ sufficiently small such that

$$
\beta_{2}^{1} \cup \widehat{f}^{N_{1}} \beta_{2}^{1} \cap \mathcal{C}_{-2}=\emptyset .
$$


Let $\beta^{1}=\beta_{1}^{1} \cdot \beta_{2}^{1}$. The arc $\beta^{1}$ satisfies items 1 and 2 .

Define $\beta^{2}$ as the subarc of $\widehat{f}^{-N_{1}} \beta$ going from $\beta^{1}(1)$ to $\widehat{f}^{-N_{1}} \lambda(1) \in \mathcal{C}_{-2}$. The arc $\beta^{2}$ then satisfies item (3) from the lemma. Letting $t_{1}=0$ we obtain that $t_{1}$ and $\beta^{2}$ satisfy item (4).

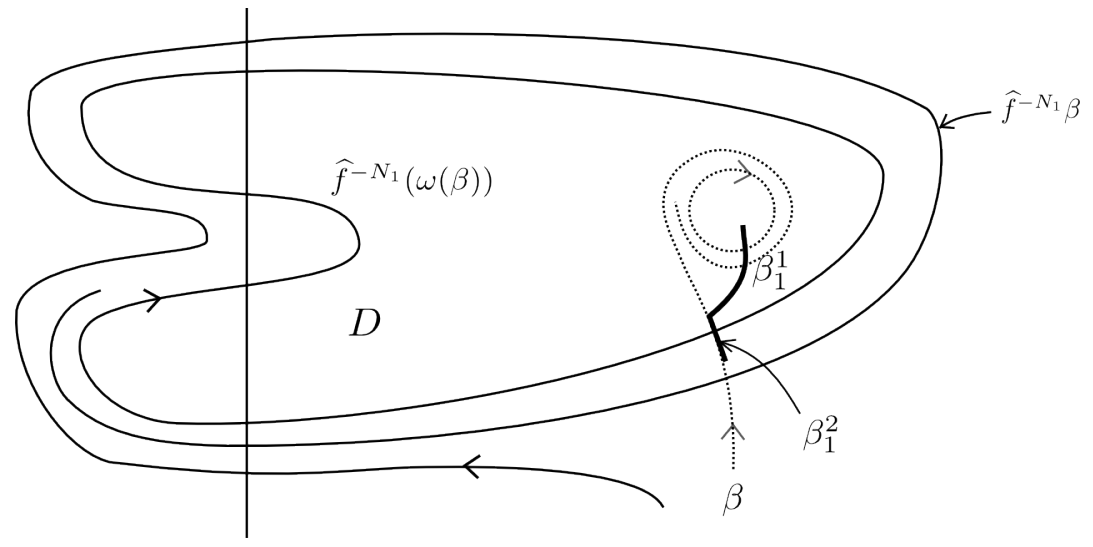

Figure 23: Illustration of Case 4.

Proof of Claim 4.46 As $\omega(\beta)$ contains no singularities, we must have $\alpha(\beta) \cap \omega(\beta)=\emptyset$. Suppose first that $\alpha(\beta)$ contains singularities, and denote the set of such singularities by $S$. As $S \subset$ $\mathbf{R}^{2} \backslash \operatorname{Fill}(\omega(\beta))$, and as $\widehat{f}$ is isotopic to the identity, $S \subset \mathbf{R}^{2} \backslash \widehat{f}^{-N_{1}}(\operatorname{Fill}(\omega(\beta)))$, which implies that $\alpha(\beta) \nsubseteq \widehat{f}^{-N_{1}}(\operatorname{Fill}(\omega(\beta)))$, and then $\beta \nsubseteq \widehat{f}^{-N_{1}} \operatorname{Fill}(\omega(\beta))$.

Now suppose that $\alpha(\beta)$ does not contain singularities. In this case there is a non-empty set $S$ of singularities contained in $\operatorname{int}(\operatorname{Fill}(\alpha(\beta)))$. Also, $S \subset \mathbf{R}^{2} \backslash \widehat{f}^{-N_{1}}(\operatorname{Fill}(\omega(\beta)))$. If $\widehat{f}^{-N_{1}}(\operatorname{Fill}(\omega(\beta)))$ contained $\beta$, it would also contain the loop $\alpha(\beta)$, and as $S \subset \operatorname{int}(\operatorname{Fill}(\alpha(\beta)))$, we would have that $\widehat{f}^{-N_{1}}(\operatorname{Fill}(\omega(\beta)))$ is not simply connected, which is a contradiction.

This finishes the proof of Lemma 4.43 .

\subsubsection{Complementary cases.}

4.7.3.1 None of the sets $\alpha(\gamma)$ and $\omega(\gamma)$ is a singularity and at least one of the sets $\alpha(\beta)$ and $\omega(\beta)$ is a singularity.

Recall that in this case, the arc $\lambda$ was defined in 4.6 .2 as $\lambda_{1} \cdot \lambda_{2}$, where $\lambda_{1}$ is straight horizontal, going leftwards from a singularity contained in Fill $(\omega(\gamma))$ to a point of $\widehat{f}^{N_{1}}(\gamma)$, and $\lambda_{2}$ is a subarc of $\widehat{f}^{N_{1}} \gamma$ going from $\lambda_{1}(1)$ to a point $z \in \widehat{f}^{N_{1}} \gamma \cap \mathcal{C}_{0}$ such that $\left.\lambda_{2}\right|_{[0,1)} \subset L\left(\mathcal{C}_{0}\right)$. Also, $\beta \subset \mathcal{C}_{0}$ was defined as the leaf of $\widehat{\mathcal{F}}$ such that $\lambda(1) \in \beta$.

Without loss of generality, we assume that $\omega(\beta)$ consists of a singularity $s$. Extend $\beta$ to $(0,1]$ as $\beta(1)=s$, and let $\beta^{1} \subset \beta$ be the subarc of $\beta$ going from $\lambda(1)$ to $\beta(1)=s$. Let

$$
t_{2}=\min \left\{t \in(0,1] \backslash U_{\widehat{f}^{-N_{1}} \beta^{1}}: \widehat{f}^{-N_{1}} \beta^{1}(t) \in \mathcal{C}_{0}\right\},
$$

(cf. Definition 4.39 ) and

$$
t_{3}=\max \left\{t \in\left[0, t_{2}\right): \widehat{f}^{-N_{1}} \beta^{1}(t) \in \mathcal{C}_{-2}\right\} .
$$


With the same arguments from Section 4.7.1, one can prove that if

$$
\delta=\left.\widehat{f}^{-N_{1}} \beta^{1}\right|_{\left[t_{3}, t_{2}\right]}
$$

(reparametrized to [0,1]), then $\delta$ leaves a leaf $\delta_{1} \subset \mathcal{C}_{-2}$ of $\widehat{\mathcal{F}}$ in $t=0$ by the right, $\operatorname{int}(\delta) \subset$ $R\left(\mathcal{C}_{-2}\right) \cap L\left(\mathcal{C}_{0}\right)$, and either $\delta$ arrives in a leaf $\delta_{2} \subset \mathcal{C}_{0}$ of $\widehat{\mathcal{F}}$ in $t$ ? 1 by the right, or $\delta(1)=s \in$ $\operatorname{sing}(\widehat{\mathcal{F}})$, and then $\delta$ satisfies items 1 to 4 from Lemma 4.25 .

4.7.3.2 None of the sets $\alpha(\beta)$ and $\omega(\beta)$ is a singularity and at least one of the sets $\alpha(\gamma)$ and $\omega(\gamma)$ is a singularity.

Without loss of generality, assume that $\omega(\gamma)$ consists of a singularity $s$. Extend $\gamma$ to $(0,1]$ as $\gamma(1)=s$. In this case, the $\operatorname{arc} \beta \subset \mathcal{C}_{0}$ was defined in $\$ 4.6 .2$ as the leaf of $\widehat{\mathcal{F}}$ that contains $\gamma\left(t_{*}\right)$, where

$$
t_{*}=\max \left\{t \in(0,1): \widehat{f}^{N_{1}} \gamma(t) \in \mathcal{C}_{0}\right\},
$$

and the arc $\lambda$ was defined as $\left(\left.\widehat{f}^{N_{1}} \gamma\right|_{\left[t_{*}, 1\right]}\right)^{-1}$.

The proof of Lemma 4.43 unmodified, gives us two $\operatorname{arcs} \beta^{1}$ and $\beta^{2}$ satisfying the conclusions of that lemma. Let $\Gamma=f^{-N_{1}} \lambda \cdot\left(\beta^{2}\right)^{-1} \cdot\left(\beta^{1}\right)^{-1}$ and define

$$
t_{2}=\min \left\{t \in(0,1] \backslash U_{\Gamma}: \Gamma(t) \in \mathcal{C}_{0}\right\},
$$

(cf. Definition 4.39), and

$$
t_{3}=\max \left\{t \in\left[0, t_{2}\right): \Gamma(t) \in \mathcal{C}_{-2}\right\} .
$$

The same arguments from Section 4.7.2 give us that if

$$
\delta=\left.\Gamma\right|_{\left[t_{3}, t_{2}\right]}
$$

(reparametrized to $[0,1]$ ), then the $\operatorname{arc} \delta$ satisfies the conclusions of Lemma 4.25

\section{References}

[Atk76] G. Atkinson, Recurrence of cocycles and random walks, Journal of the London Mathematical Society 13 (1976), no. 2, 486-488.

[BCJR09] F. Béguin, S. Crovisier, T. Jäger, and F. Le Roux, Denjoy constructions for fibered homeomorphisms of the torus, Trans. Amer. Math. Soc. 361 (2009), no. 11, 58515883.

[Cai51] S. Cairns, An elementary proof of the Jordan-Schoenflies theorem, Proc. of the Amer. Math. Soc. 91 (1951), no. 2, 860-867.

[Cal05] P. Le Calvez, Une version feuilletée équivariante du théorème de translation de Brouwer, Publications Mathématiques de l'IHÉS 102 (2005), no. 1, 1-98.

[Dav86] R. J. Daverman, Decompositions of manifolds, vol. 124, Academic Press Inc., Orlando, FL, 1986.

[Dáv13] P. Dávalos, On torus homeomorphisms whose rotation set is an interval, Mathematische Zeitschrift (2013), DOI 10.1007/s00209-013-1168-3. 
[Fay02] B. Fayad, Weak mixing for reparametrized linear flows on the torus, Ergodic Theory and Dynamical Systems 22 (2002), 187-201.

[FM90] J. Franks and M. Misiurewicz, Rotation sets of toral flows, Proc. Amer. Math. Soc. 109 (1990), no. 1, 243-249.

[Fra89] J. Franks, Realizing rotation vectors for torus homeomorphisms, Transactions of the American Mathematical Society 311 (1989), no. 1, 107-115.

[Fra95] - The rotation set and periodic points for torus homeomorphisms, Dynamical Systems \& Chaos (Aoki, Shiraiwa, and Takahashi, eds.), World Scientific, Singapore (1995), 41-48.

[Fur61] H. Furstenberg, Strict ergodicity and transformation of the torus, Amer. J. of Math. 83 (1961), 573-601.

[GKT12] N. Guelmanm, A. Koropecki, and F. A. Tal, A caracterization of annularity for area preserving toral homeomorphisms, eprint arXiv:1211.5044v1 (2012).

[Gut79] C. Gutiérrez, Smoothing continuous flows and the converse of Denjoy-Schwartz theorem, An. Acad. Brasil. Ciênc. 51 (1979), no. 4, 581-589.

[Han89] M. Handel, Periodic point free homeomorphisms of $\mathbb{T}^{2}$, Proc. of the Amer. Math. Soc. 107 (1989), no. 2, 511-515.

[Han90] - The rotation set of a homeomorphism of the annulus is closed, Communications in Mathematical Physics 127 (1990), 339-349.

[Jäg09a] T. Jäger, The concept of bounded mean motion for toral homeomorphisms, Dyn. Sys. 24 (2009), no. 3, 277-297.

[Jäg09b] _ Linearisation of conservative toral homeomorphisms, Invent. Math. 176 (2009), no. 3, 601-616.

[Jau13] O. Jaulent, Existence d'un feuilletage positivement transverse à un homeomorphism de surface, arXiv:1206.0213 (2013).

[KK08] A. Kocksard and A. Koropecki, Free curves and periodic points for torus homeomorphisms, Ergodic Theory \& Dynamical Systems 28 (2008), 1895-1915.

[KK09]__ A mixing-like property and inexistence of invariant foliations for minimal diffeomorphisms of the 2-torus, Proc. of the Amer. Math. Soc. 137 (2009), no. 10, 3379-3386.

[KT] A. Koropecki and F. A. Tal, Strictly toral dynamics, To appear in Invent. Math.

[KT12a] - Area preserving irrotational diffeomorphisms of the torus with sublinear diffusion, To appear in Proc. Amer. Math. Soc. (2012).

[KT12b] _ Bounded and unbounded behaviour for area-preserving rational pseudorotations, Preprint, arXiv:1207.5573v3 (2012).

[Kwa92] J. Kwapisz, Every convex polygon with rational vertices is a rotation set, Erg. Theory and Dyn. Sys. 12 (1992), 333-339. 
[Kwa95]__ A toral diffeomorphism with a nonpolygonal rotation set, Nonlinearity 8 (1995), no. 4, 461-476.

[LM91] J. Llibre and R. S. Mackay, Rotation vectors and entropy for torus homeomorphisms isotopic to the identity, Ergodic Theory \& Dynamical Sistems 11 (1991), 115-128.

[Mat97] S. Matsumoto, Rotation sets of surface homeomorphisms, Bol. Soc. Bras. Mat. 28 (1997), no. 1, 89-101.

[Mos65] J. Moser, On the volume elements on a manifold, Trans. of the Amer. Math. Soc. 120 (1965), no. 2, 286-294.

[MZ89] M. Misiurewicz and K. Ziemian, Rotation set for maps of tori, Journal of the London Mathematical Society 40 (1989), no. 2, 490-506.

[NS89] V. V. Nemitskii and V. V. Stepanov, Qualitative theory of differential equations, Courier Dover Publications, 1989.

[Poi52] H. Poincaré, Oeuvres complètes, Gauthier-Villars, Paris, 1952.

[Pol92] M. Pollicott, Rotation sets for homeomorphisms and homology, Trans. Amer. Math. Soc. 331 (1992), no. 2, 881-894.

[Sch57] S. Schwartzmann, Assymptotic cycles, Ann. of Math. 66 (1957), no. 2, 270-284.

[Sol45] G. Solntzev, On the asymptotic behaviour of integral curves of a system of differential equations, Bull. Acad. Sci. URSS. Sér. Math. [Izvestia Akad. Nauk SSSR] 9 (1945), $233-240$.

[Tal13] F. A. Tal, On non-contractible periodic orbits for surface homeomorphisms, e-print: arXiv:1307.1664 (2013).

[Whi33] H. Whitney, Regular families of curves, Ann. of Math. 34 (1933), no. 2, 244-270.

[Whi41]__ On regular families of curves, Bull. Amer. Math. Soc. 47 (1941), 145-147.

Instituto Tecnológico y de Estudios Superiores de Occidente, Periférico Sur Manuel Gómez Morín 8585, C.P. 45604, Tlaquepaque, Jalisco, MéXico.

mailto: pablod@iteso.mx 